\title{
WestVirginiaUniversity
}

THE RESEARCH REPOSITORY @ WVU

Graduate Theses, Dissertations, and Problem Reports

2012

\section{Quantification of Mixing in Microchannels}

\author{
Vijaymaran Manickam \\ West Virginia University
}

Follow this and additional works at: https://researchrepository.wvu.edu/etd

\section{Recommended Citation}

Manickam, Vijaymaran, "Quantification of Mixing in Microchannels" (2012). Graduate Theses,

Dissertations, and Problem Reports. 679.

https://researchrepository.wvu.edu/etd/679

This Thesis is protected by copyright and/or related rights. It has been brought to you by the The Research Repository @WVU with permission from the rights-holder(s). You are free to use this Thesis in any way that is permitted by the copyright and related rights legislation that applies to your use. For other uses you must obtain permission from the rights-holder(s) directly, unless additional rights are indicated by a Creative Commons license in the record and/ or on the work itself. This Thesis has been accepted for inclusion in WVU Graduate Theses, Dissertations, and Problem Reports collection by an authorized administrator of The Research Repository @ WVU. For more information, please contact researchrepository@mail.wvu.edu. 
Quantification of Mixing in Microchannels

by

Vijaymaran Manickam

Thesis submitted to the

Benjamin M. Statler

College of Engineering and Mineral Resources

at

West Virginia University

In partial fulfillment of the requirements for the degree of

Master of Science

In

Mechanical Engineering

\author{
Ismail B. Celik, Ph.D, Chair \\ Wade Huebsch, Ph.D. \\ Yuxin Liu, Ph.D.
}

Mechanical and Aerospace Engineering Department

Morgantown, West Virginia

(2012)

Keywords: Microchannels, streamtrace, mixing, CFD 


\section{ABSTRACT \\ Quantification of Mixing in Microchannels}

\section{Vijaymaran Manickam}

Microchannels are used for delivery of two-or more fluids for multiple purposes, such as drug delivery, where good mixing is desired in a very short time (or distance). For this purpose, many design options are being proposed in the literature. For example herringbone baffles at the bottom of a rectangular channel are commonly proposed to enhance mixing in a drug delivery device. To assess the effectiveness of such devices many experiments need to be performed thus increasing the design cycle time and the cost involved. Computational fluid dynamics (CFD) can and is being used to shorten this design cycle by performing parametric analysis; however, due to numerical errors it is also necessary to verify and then validate the numerical models to ensure that the predictions are indeed accurate. In this study a recently developed microchannel is analyzed using CFD to determine its mixing effectiveness. There are two common ways of assessing the degree of mixing: (a) via calculation of a passive scalar transport equation, (b) by following fluid particle trajectories and calculating the statistics. The first approach suffers from presence of numerical diffusion. The second approach is usually used to only obtain qualitative information rather than quantitative assessment. In this study we explore both approaches and reconcile both of these in terms of extracting quantitative information. Furthermore we assess the results of simulations using both approaches to determine a mixing index that provides directly a measure of mixing quality. 


\section{Acknowledgements}

It has been an absolute pleasure working under the supervision of Dr. Ismail Celik. There have been times when I have been completely lost only for him to step in and clear out the way ahead. He would often enquire about my academic and social well-being which greatly increased my respect for him. I wish to take this opportunity to thank him for his continued support and encouragement throughout my MS program at WVU. I am also very grateful to my fellow CFD\&AMP lab partners who have all been extremely kind and helpful during challenging times. I have also immensely come to enjoy each one of them as earnest human beings who were able to relate and sympathize with our lot as hardworking graduate students - they have often provided a pleasant time-out amidst seasons of intense stress.

To say my family was a great support would be an understatement. My umpteen phone calls meant to vent out my frustrations and joys have always been attended to with the utmost care and outer-worldly patience. They have sacrificed a lot for my well-being and I am immensely happy to be able to put a smile on their faces.I am certain there is a great someone "The Divine" who has been very good to me - watching over, guiding, and keeping me in good health during my stay at WVU; and I wish to acknowledge their presence. 


\section{Contents}

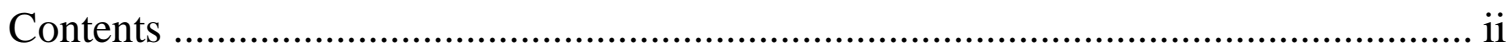

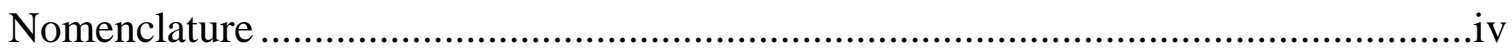

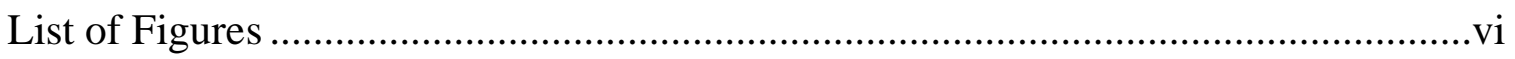

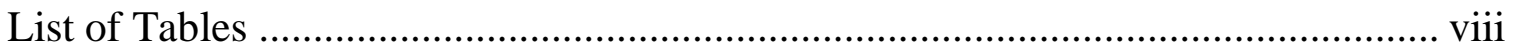

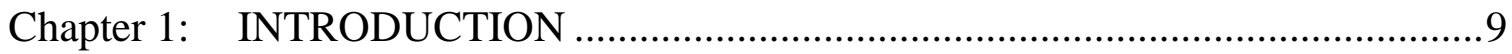

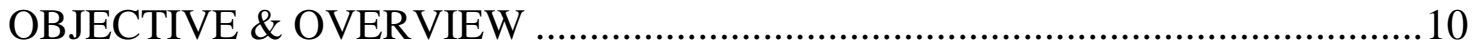

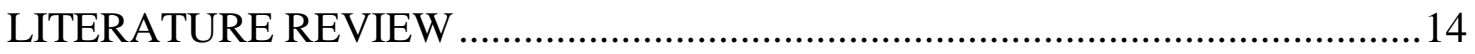

Chapter 2: QUANTIFICATION METHOD ………….......................................16

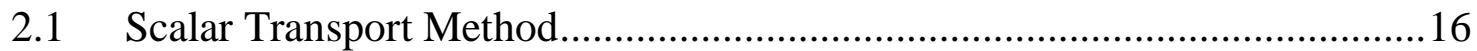

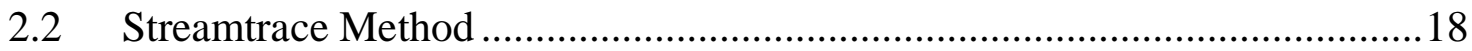

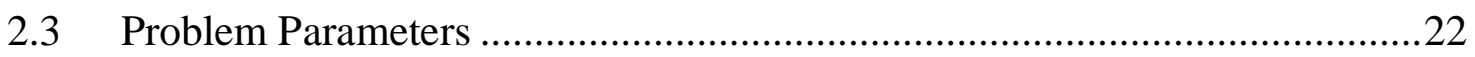

2.4 Mesh and Computational Information .................................................22

Chapter 3: STAGGERED HERRINGBONE GEOMETRY RESULTS .................24

3.1 Staggered Herringbone Geometry ………………................................2

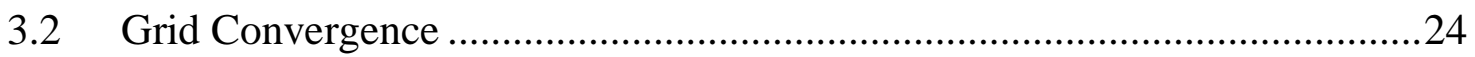

3.3 Comparison of Different Numerical Schemes ...............................................26

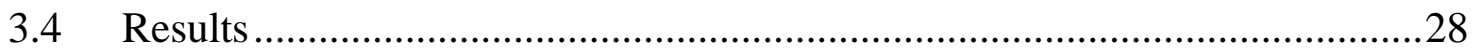

Chapter 4: MULTIPLE BEND GEOMETRY RESULTS ..........................................36

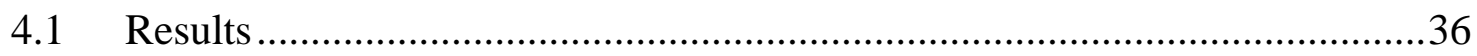

Chapter 5: RESOLVING THE MULTIPLE BEND GEOMETRY .........................43

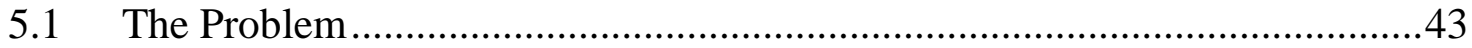

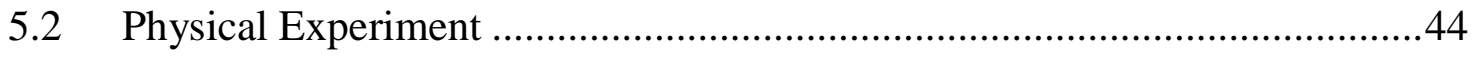

5.3 Re-solving the scalar transport equation ....................................................50 


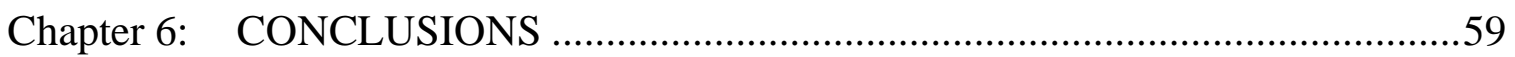

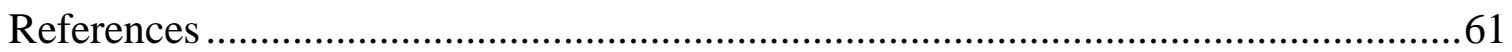

APPENDIX A : Solving Diffusion Equation Numerically .......................................65

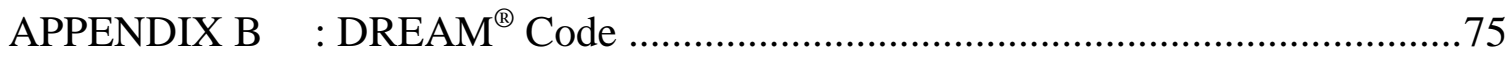

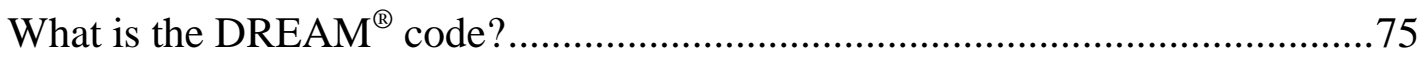

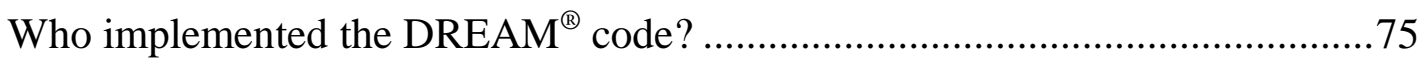

What is the objective of using DREAM ${ }^{\circledR}$ here?..................................................75

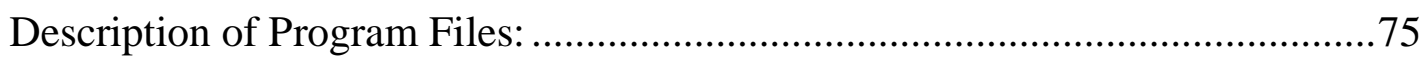

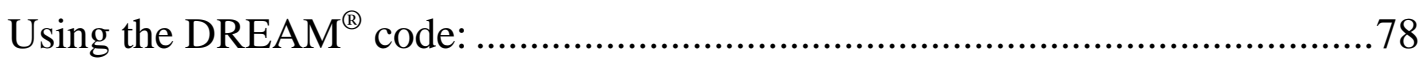

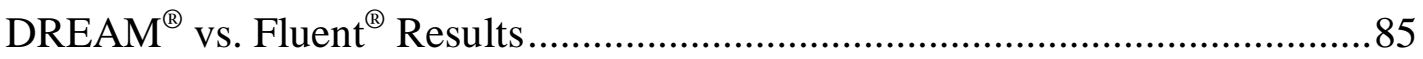




\section{Nomenclature}

Letters

i

j

1

M

$\mathrm{N}$

P1

P2

$\begin{array}{ll} & {[\mathrm{Pa}]} \\ & {\left[\mathrm{s}^{-1}\right]} \\ \vec{V} & {\left[\mathrm{~m} \mathrm{~s}^{-1}\right]} \\ \overrightarrow{v(x)} & {\left[\mathrm{m} \mathrm{s}^{-1}\right]} \\ & {[\mathrm{m}]}\end{array}$

$[-]$

[-]

[Pa]

$\left.\mathrm{s}^{-1}\right]$$$
\left.\mathrm{m} \mathrm{s}^{-1}\right]
$$

m] grid index along transverse axis

grid index along vertical axis

diffusion constant

mass of fluid-1

Mass of fluid-2

grid divisions along transverse axis

grid divisions along vertical axis

points representing fluid 1 in a bin

points representing fluid 2 in a bin

total points representing fluid 1 in crosssection

Total points representing fluid 2 in a crosssection

pressure

time

velocity vector

velocity vector at location $\mathrm{x}$

position vector 
Greek symbols

$\begin{array}{ll}{[-]} & \text { mass fraction } \\ {[\mathrm{Pa} \mathrm{s}]} & \text { viscosity } \\ {\left[\mathrm{kg} \mathrm{m}^{-3}\right]} & \text { density }\end{array}$




\section{List of Figures}

Figure 1-1: Staggered Herringbone geometry from literature ${ }^{[5]}$..............................10

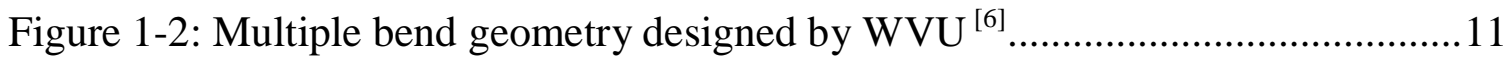

Figure 1-3: Alternate view of multiple bend geometry designed by WVU ${ }^{[6]} \ldots \ldots \ldots \ldots . . .12$

Figure 2-1: Simple Streamtrace Example .............................................................21

Figure 3-1: HGB Channel Grid Convergence ………………………………….....24

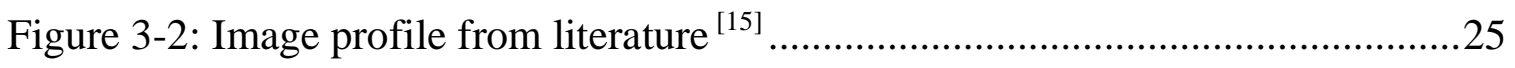

Figure 3-3: HGB geometry scheme convergence ...............................................26

Figure 3-4: Staggered Herringbone geometry data at $0 \mathrm{~mm}$.........................................31

Figure 3-5: Staggered Herringbone geometry data at $2.09 \mathrm{~mm}$..................................32

Figure 3-6: Staggered Herringbone geometry data at $5.39 \mathrm{~mm}$....................................33

Figure 3-7: Staggered Herringbone geometry data at $7.59 \mathrm{~mm}$....................................34

Figure 3-8: Staggered Herringbone geometry data at $10.89 \mathrm{~mm}$.................................35

Figure 4-1: Multiple bend channel data at bends 1, and 2 ........................................... 37

Figure 4-2: Multiple bend channel data at bends 3, and 4 ..........................................38

Figure 4-3: Multiple bend channel data at bends 5, and 6 .........................................39

Figure 4-4: Multiple bend channel, mass-fraction profiles...........................................41

Figure 4-5: Multiple bend channel; Contour vs. Qualitative snapshot ${ }^{[6]}$....................42

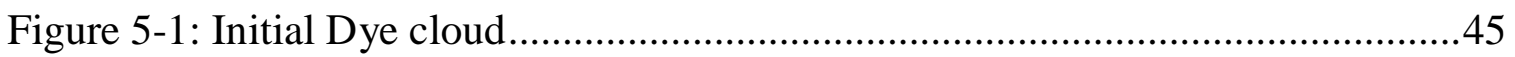

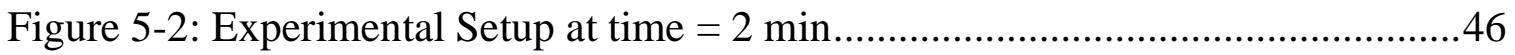

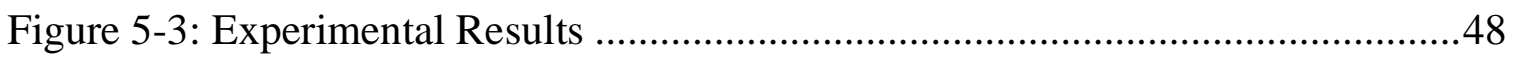

Figure 5-4: Experimental vs. Numerical results ....................................................49

Figure 5-5 - Qualitative vs. Mass-Fraction for Multiple-bend-geometry ……............52

Figure 5-6 - Mass-Fraction data for varying diffusivities ............................................53

Figure 5-7 - Inlet profile for different diffusivities ...................................................54

Figure 5-8 - Outlet profile for different diffusivities...............................................55

Figure 5-9: Matching Reynolds number with Experiments and Simulations ..............57

Figure 5-10: Flow with $\mathrm{Re}=1,25$, and 50 ; with diffusivity $=5.5 \mathrm{e}-7 \mathrm{~m}^{2} / \mathrm{s} \ldots \ldots \ldots \ldots \ldots \ldots . . .58$ 
Figure A-1: Diffusivity Geometry and initial/boundary conditions ...........................66

Figure A-2: Volume elements for discretization ..................................................66

Figure A-3: Radius vs. Density at time 720 seconds for diffusivity $=1 \mathrm{e}-7 \mathrm{~m}^{2} / \mathrm{s}$.......68

Figure A-4: Radius vs. Lambda at time 720 seconds for diffusivity $=1 \mathrm{e}-7 \mathrm{~m}^{2} / \mathrm{s}$........69

Figure B-1: A brief flow chart of the DREAM ${ }^{\circledR}$ code obtained from [24] ...................77

Figure B-2: (i) and (ii) represents the mesh generated by DREAM ${ }^{\circledR}$.........................79

Figure B-3: DREAM vs. Fluent; axial profile ……….............................................. 81

Figure B-4: DREAM vs. Fluent; Vertical Velocity …………………........................8 82

Figure B-5: DREAM vs. Fluent; transverse velocity................................................83

Figure B-6: DREAM vs. Fluent, flow-rates conservation ...........................................84 


\section{List of Tables}

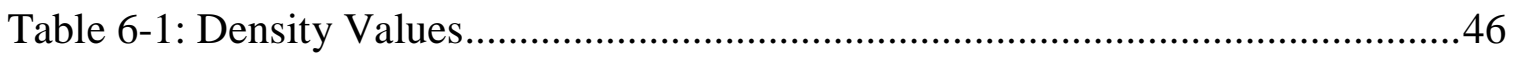

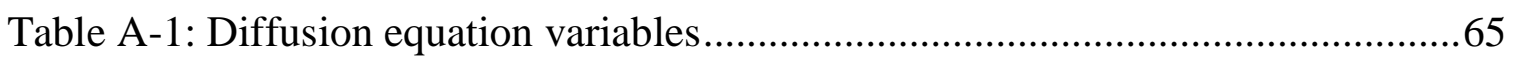

Table A-2: Detailed Numerical Method Procedure ……….........................................71

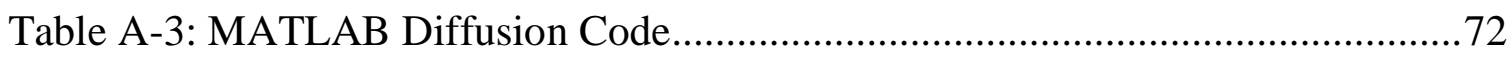

Table B-1: Brief component overview of the DREAM ${ }^{\circledR}$ code ……….........................76

Table B-2: Difference between Dream and Fluent .................................................... 80

Table B-3: HGB geometry internal wall code .......................................................... 87

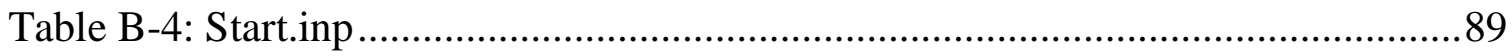




\section{Chapter 1: INTRODUCTION}

Microchannels and other microfluidic systems have become prevalent in many biotechnology and biomechanical applications including DNA analysis ${ }^{[1]}$, cell sorting ${ }^{[2]}$, chemical reactions ${ }^{[3]}$ and the transmission of small amount of fluids ${ }^{[4]}$. Some of these applications require the fluids passing through the microfluidic systems to be mixed in an efficient manner such as the homogenization of solutions containing reagents used in a chemical reaction ${ }^{[5]}$.

In microchannels, the characteristic dimensions of the geometry are very small (on the order of $100 \mu \mathrm{m}$ ) which causes the flows to be characterized by low values of the Reynolds number $<100$ (based on hydraulic diameter) ${ }^{[5]}$. This results in a predominantly laminar flow regime in which there is no inherent tendency for flows to mix for uniaxial flows. Also, even at the micro scale, mixing due to molecular diffusion is slow with respect to the convective mixing of the fluid along the channel. Because of these phenomena, typical mixing lengths that are impractical for most applications occur $(>>1 \mathrm{~cm})^{[5]}$. Therefore, it is desirable to induce mixing by other means.

In order to reduce the length of channel required for mixing, transverse velocity components must be introduced (chaos induction). Transverse flow components create "folds" of volumes of fluid in the cross section of the channel and stretch these volumes so that diffusion between volumes can occur more rapidly ${ }^{[5]}$. Theoretically there are an infinite number of complex designs for inducing chaos using geometric blocks. However, design limitations do exist due to manufacturability, cost and testing difficulties. It is therefore preferred that a channel should be designed to be both simple and efficient. 


\section{OBJECTIVE \& OVERVIEW}

In this study, mixing of two fluids in two different microchannel geometries is studied using computational fluid dynamic (CFD) techniques. The first geometry has been developed and studied in detail previously and is referred to as a staggered herringbone mixer (HGB); see Figure 1-1.

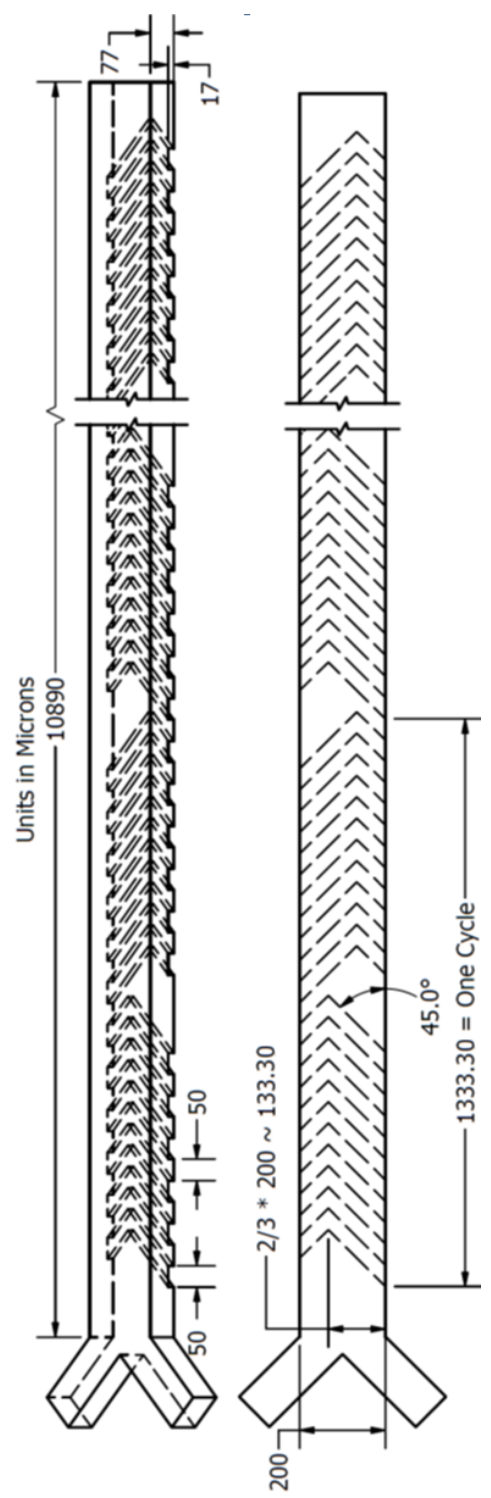

Figure 1-1: Staggered Herringbone geometry from literature ${ }^{[5]}$ 


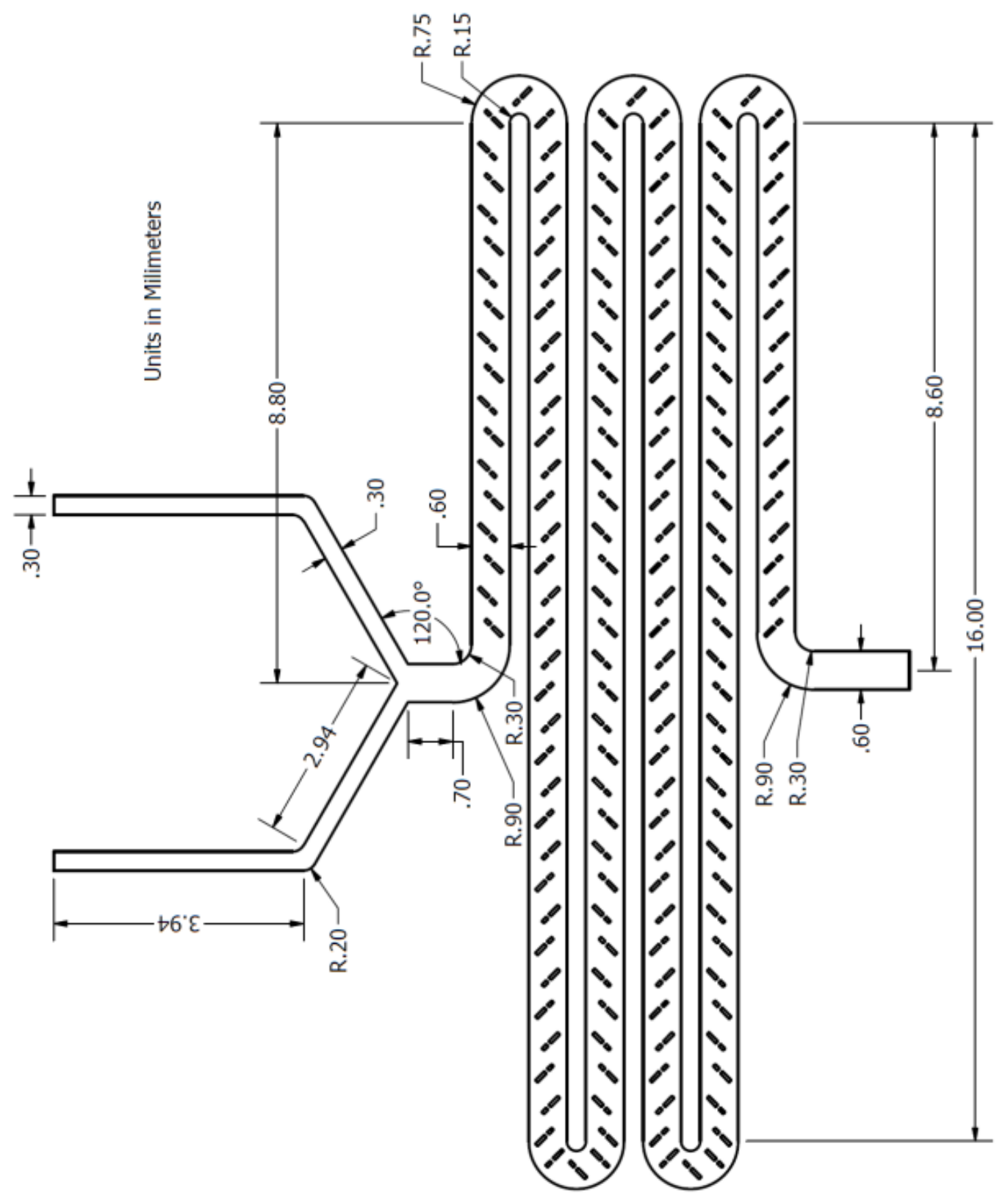

Figure 1-2: Multiple bend geometry designed by WVU ${ }^{[6]}$ 


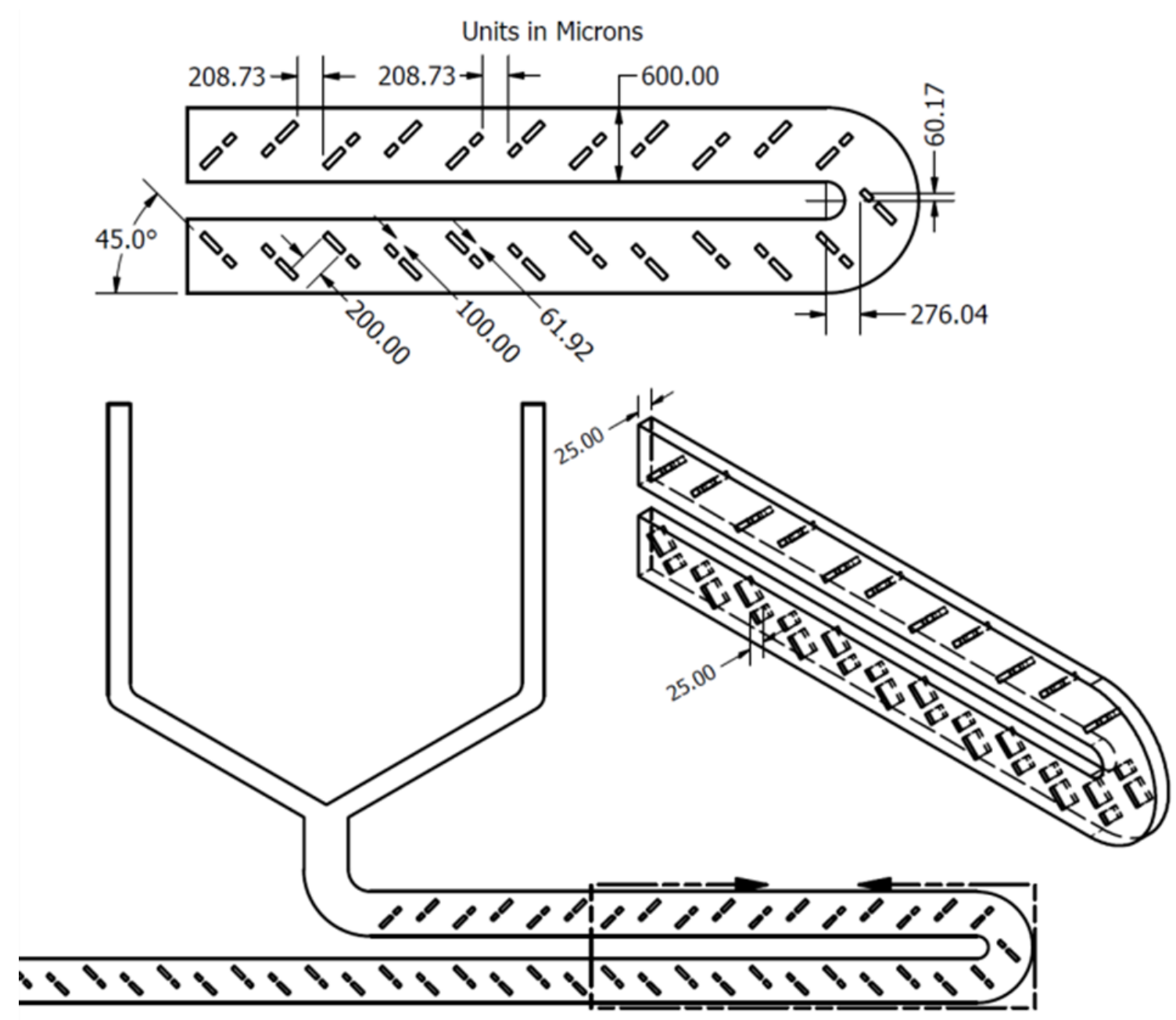

Figure 1-3: Alternate view of multiple bend geometry designed by $\mathrm{WVU}{ }^{[6]}$ 
The dimensions of the geometry are similar to that reported in literature ${ }^{[5] .}$ In this geometry mixing is achieved by using baffles protruding into the flow-channel. These structures stretch and fold the fluids into each other. The second geometry was recently developed at West Virginia University (WVU) and is yet to undergo extensive testing. This geometry will be referred to as the multiple-bend geometry. Rather than utilizing a grooved channel floor, this channel design has structures which protrude up through the entire thickness of the channel; see Figure 1-2, and Figure 1-3.

Whenever a new design is proposed, the effective mixing induced and the anticipated improvement compared to previous designs should be assessed. This is usually done by introducing dye or a similar agent into one fluid and observing the degree of mixing virtually. Such experiment only provides qualitative information unless, the diffusivity of the agent in the fluid is precisely known and is much smaller than the self-diffusion coefficient of the two streams of fluid being considered. On the other hand the use of computational fluid dynamics (CFD) provides the alternative of setting the diffusivity of the dye to any lesser value. However, CFD simulations have the disadvantage of introducing artificial diffusion arising from numerical errors. There is a need for new assessment techniques whereby these numerical errors are minimized.

The objective of this study is to test the validity of a newly developed quantification method to assess mixing based on a similar procedure reported in literature ${ }^{[7] .}$ The results obtained by utilizing the new method are compared against the results from traditional methods (discretized Governing Equation) and experiments in order to establish its credibility. Finally, an attempt is made to analyze a recently developed geometry using the new method. 


\section{LITERATURE REVIEW}

Mixing is one of the most fundamental functions that need to be accomplished in industry. Researchers have long strived to implement efficient designs that contribute to effective mixing. The strides that have been made in improving industry grade large scale mixers have been immense. In fact, the development of quality mixers could potentially be termed one among a fleet of other developments that contributed to the industrial revolution. Taking a moment to consider all the power-plants currently in use will bear witness to this testimony.

Despite the very encouraging advancement in the heavy-duty industry, mixing in micro-scale mixers have been a relatively recent development. New models with innovative designs have been proposed only in the last decade ${ }^{[8]}$. With the relevant hardware becoming available only in the recent past, it is not surprising that the theory behind quantifying mixing (in micro-mixers) is also a very recent development.

Chemical engineers have been dealing with micro-mixers for a marginally longer period (since the 1980s) ${ }^{[9]}$. Traditionally two approaches exist to quantify mixing. The first approach involved determining an analytical solution for each unique case. Even though this seemed plausible for a few simple geometries, it was highly impractical ${ }^{[8]}$. In addition to being very tedious, analytical solutions to fluid flow with complex-physics are extremely rare, hence difficult.

The second approach was a purely numerical procedure. The numerical method was straight forward and rendered good results. However, owing to the computational demands of the procedure, its use was limited to a few models that demonstrated promising results in the experimental phase.

Researchers were considering alternatives to the numerical procedure that would essentially reduce the computational demands of the process. One innovative option was to develop a visualization technique utilizing streamtraces ${ }^{[7]}$. This was innovative 
because the velocity field alone was utilized to generate mixing-data. This mixingdata was represented by particles dispersed on a 2D plane.

Dispersion patterns on 2D maps have been used by physicists to quantify mixing with very good success - the 2D graphs so obtained are termed Poincare maps ${ }^{[10] .}$ The Poincaré maps received excellent support from academic communities and prompted its use to quantify mixing in the engineering field ${ }^{[11]}$.

Coming back to current study, the 2D maps generated as described above may be analyzed by a number of statistical manipulations. It may be noted that a detailed discussion regard the procedure is included in the subsequent sections. Many different statistical variations have been proposed and tested with good results for microchannels ${ }^{[12]}$.

However, the statistical method utilized in this study is unique and simple. The uniqueness arises from utilizing qualitative data (2D maps) to develop quantitative data. There have been discussions on how this procedure failed to describe dependable results for complicated geometries ${ }^{[13]}$. In this study a simple geometry that has been experimentally validated is utilized to generate quantitative data from qualitative information. Furthermore, the same procedure is utilized on a more complex geometry that was developed at WVU. The findings are clearly documented and discussed in-detail. 


\section{Chapter 2: QUANTIFICATION METHOD}

Information regarding two parameters is required in order to quantify the extent and efficiency of mixing. Any parameter that indicates the concentrations of the individual fluids after mixing can be used to assess the extent of mixing. Mass Fraction values are an excellent measure for this purpose. A uniform mass fraction of each fluid in the mixture across the channel cross-section will imply thorough mixing. As an example, in real life experiments involving microchannels, dye-intensity studies are employed to generate qualitative mixing data. The geometry is typically constructed using glass or other transparent media. The inlet fluids are tainted with dyes of varying intensity and allowed to flow through the channel. Snapshots of the channel at different cross-sections along the channel length are generated. This information is thereafter utilized to generate mass-fraction like values based on literature ${ }^{[5] .}$ The efficiency of mixing is typically reported in literature in terms of the time taken or distance traversed prior to thorough mixing; see source ${ }^{[7]}$. Likewise, in this study distance was used to assess mixing. Using distance as the preferred parameter will require mixing-data at different cross-sections along the channel length. For instance, the mass-fraction values at the inlet and exit of the geometry can be computed and compared to determine the extent of mixing. Having settled on the parameters required to assess mixing, the next step was to catalog the list of alternative techniques available to generate the solution data.

\subsection{Scalar Transport Method}

Solving the scalar transport equation (Eq. 1) will render mass-fraction data at any location within the geometry. The velocity field data required in the scalar transport equation is obtained by solving the Navier-Stokes equations to determine the velocity

components; see Eq. (2, 3 and 4). With the advent of commercial software packages capable of handling a wide range of fluid flow problems the task of solving these 
equations has become fairly manageable. However, there exist a number of preliminary steps that have to be performed to obtain a reliable solution. Meshing, choosing solutions methods and grid-convergence study are some of the steps that need to be assessed along the way. More about these issues will be discussed in the later sections.

Despite these methods having improved drastically in the last few decades with the introduction of commercially available solvers, there still exist some inherent challenges with the approach. For instance one big challenge arises from extensive numerical diffusion that arises in the numerical solution of scalar transport equation for mass fractions. The extent of numerical diffusion primarily depends on the solution-method and mesh-size of the geometry. However, there always exist limitations on the mesh size, and choice of solution methods due to other constraints. Hence, it may not be possible in some cases to reduce numerical diffusion errors to acceptable limits and alternative techniques are required to circumvent the problem. An excellent discussion on the common shortcomings of Eulerian approach for mixing in micro-channels can be found in literature ${ }^{[14]}$.

$$
\begin{aligned}
& \frac{\partial(\rho \Phi)}{\partial \mathrm{t}}+\nabla \cdot[\rho(\Phi) \Phi \vec{V}]=\nabla \cdot[D(\Phi) \nabla \Phi] \\
& \Phi=\frac{m 1}{(m 1+m 2)} \\
& \frac{\partial(\rho \vec{V})}{\partial t}+\rho \vec{V} \cdot \nabla(\vec{V})=-\nabla P+\nabla \cdot \mu \nabla \vec{V}
\end{aligned}
$$




$$
\nabla \cdot[\rho \vec{V}]=0
$$

\subsection{Streamtrace Method}

Over the last few years some indirect methods have been successfully used to analyze mixing in micro-channels. Some of the popular methods utilize streamtrace data. Streamtraces are trajectories of massless particles following the velocity field. Crosssections with the trajectories of these tracers cutting through them are extracted at selected locations along the channel. The distribution of the traces in the crosssections appears as point-clouds on a plane. Subsequent statistical manipulations may be carried out on this data to generate mass-fraction like values that can be used to quantify mixing. These methods are classified under the Lagrangian approach whereby equation of motion (Eq. 5) is integrated for each particle ${ }^{[14]}$. The Lagrangian nature of the setup eliminates the numerical diffusion that exists in the system arising from convective terms; see Eq. 1. However, this approach only accounts for mixing due to convection, and thus probably underestimates the total mixing which is acceptable for the current application.

The trajectory of the massless particle may be computed by integrating the equation of motion given by:

$$
\frac{d \boldsymbol{X}}{d t}=\overrightarrow{v(x)}
$$

In the current study, this exercise was carried out using the commercially available Tecplot@ post-processing tool. While performing the integration over the velocity field, Tecplot ${ }^{\circledR}$ utilizes the trapezoidal rule which is a $2^{\text {nd }}$ order accurate scheme. It 
may be noted here that a higher order scheme would potentially render more accurate results.

The above formulation will render point-cloud distribution of the stream-tracers on a plane. Various statistical analysis techniques are employed in literature to postprocess the point cloud data and quantify the mixing. The intensity of segregation index is one popular technique that is repeatedly used. In this technique the data are distributed in bins and their variation utilized to calculate mass-fraction like data ${ }^{[10]}$.

After reviewing the different techniques currently in use, a new technique has been formulated and utilized in the current study. This technique is very simple and straightforward but has been tested against experimental data to render encouraging results. As in the other methods from literature ${ }^{[10]}$, a grid of adequate size is placed on the cross-section and the points of the stramtracer cloud are collected into bins representing the grid cells they fall in. There exist two new variables which are defined below and their distributions are calculated using the stream tracer distribution on the grid. Each new variable corresponds to one of the fluids being mixed in the microchannel.

Let P1 and P2 correspond to the total number of data points representing either fluid (Fluid-1 and Fluid-2) on any given cross-section; And p1i,j and p2i,j are the number of points in any given bin $[\mathrm{i}, \mathrm{j}]$. There are $\mathrm{NxM}$ bins in a $\mathrm{N}$ by $\mathrm{M}$ grid and the following equation holds. For a simple flowchart and example describing the streamtrace method please see Error! Reference source not found. and Figure 2-1.

$$
\sum_{j=1}^{M} \sum_{i=1}^{N} p 1_{i, j}=P 1, \text { and } \sum_{j=1}^{M} \sum_{i=1}^{N} p 2_{i, j}=P 2
$$

The quality of mixing in any bin is determined by the fraction of points from one fluid in the total number of points from both fluids given by: 
$\left[\right.$ Mixing Quality $_{i, j}=\left[\frac{\mathrm{p} 1}{\mathrm{p} 1+\mathrm{p} 2}\right]_{i, j} \ldots$ for fluid 1

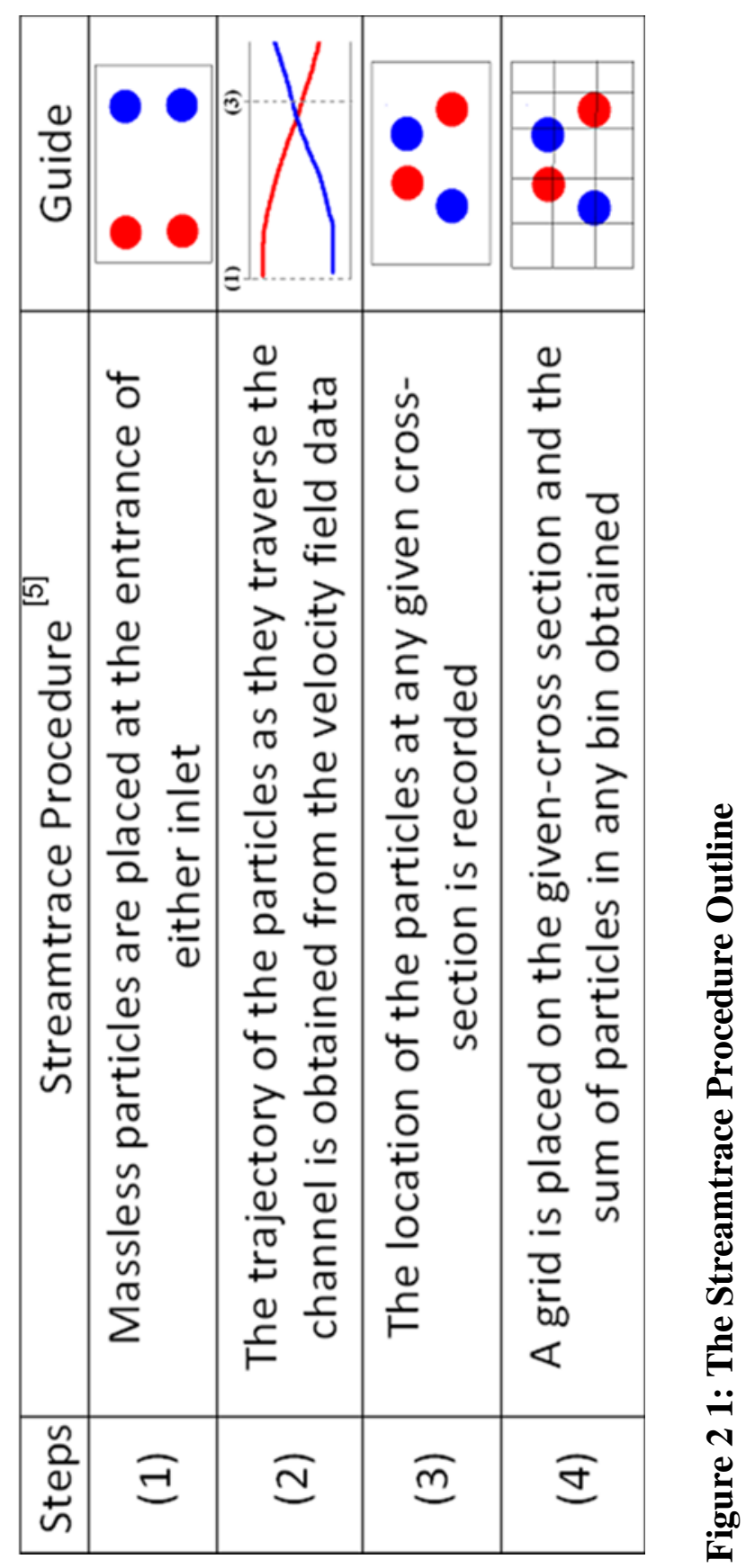




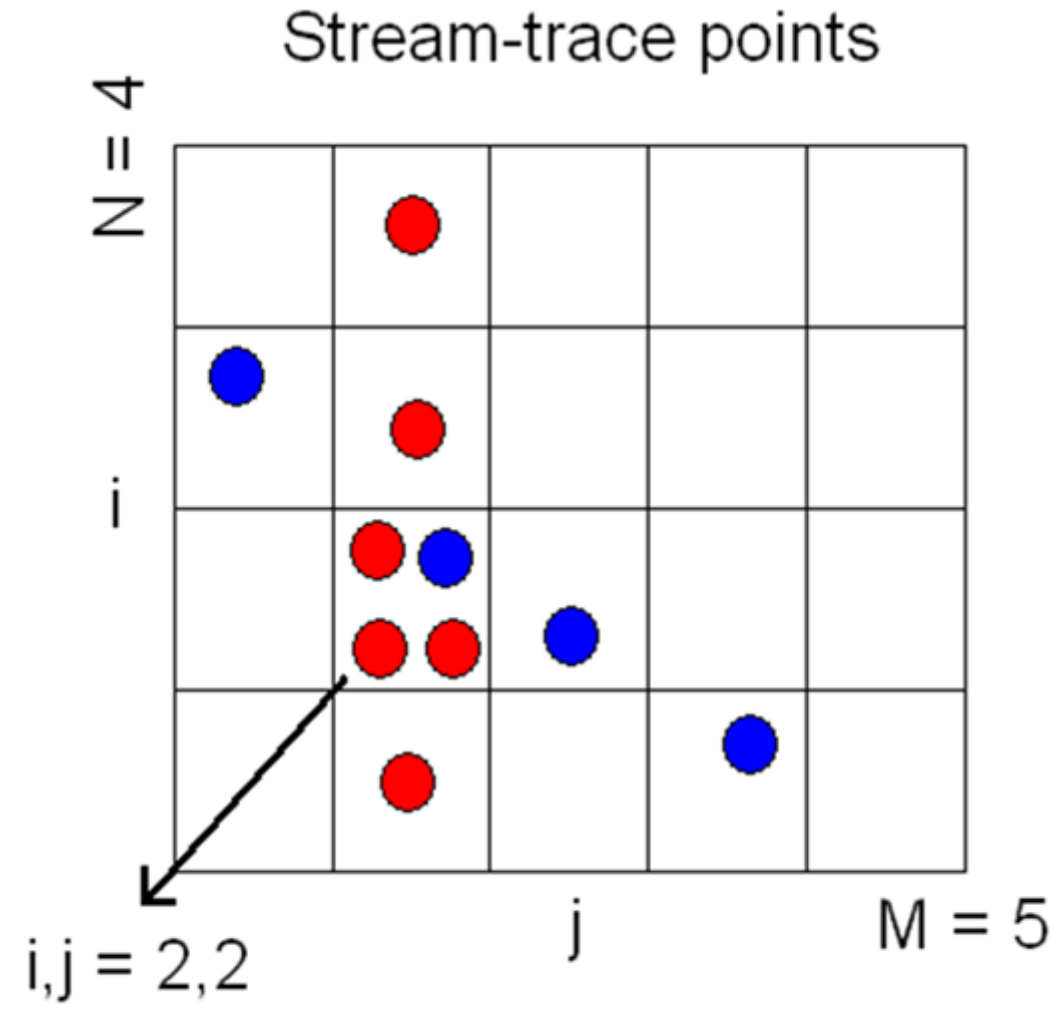

$\mathrm{P} 1=4 ; \mathrm{p} 1_{(\mathrm{i}, \mathrm{j}=2,2)}=1$; Fluid-1 is in Blue

$\mathrm{P} 2=6 ; \mathrm{p} 2_{(\mathrm{i}, \mathrm{j}=2,2)}=3$; Fluid-2 is in Red

Mass-Fraction-Like-Data_ $1_{(\mathrm{i}, \mathrm{j}=2,2)}=1 / 4=0.25$ for Blue

Figure 2-1: Simple Streamtrace Example 


\subsection{Problem Parameters}

It may be noted here that both geometries considered in this study have identical flow properties similar to that of water. However the mass diffusivity of either fluid into the other is set to about $2 \mathrm{e}-15$. At such small values mixing by diffusion is nonexistent. Consequently any mixing if at all present is solely by volume folds induced by the influence of the geometric constraints and that due to numerical diffusion. This is vital as it is now possible to analyze and compare the potential of the geometry to induce mixing. Also the Reynolds number characterizing the flow is set close to 1 . All values utilized here are from actual experiments conducted on the herringbone geometry as reported by literature ${ }^{[5]}$ and the new micro-channel geometry being tested at $\mathrm{WVU}^{[6]}$.

The computations in the study were carried out using the commercially available ANSYS FLUENT WORKBENCH utility software.

\subsection{Mesh and Computational Information}

Three meshes were created for the Herringbone geometry. The three meshes had varying number of elements so as to classify them as Fine, Medium and Coarse. The fine mesh consisted of 1,833,541 tetrahedral, and 215,580 pyramidal elements. The medium mesh consisted of 1,446,324 tetrahedral and 156,476 pyramidal elements. The coarse mesh consisted of 911,356 tetrahedral, and 77,472 pyramidal elements. The tetrahedral elements filled the interior of the geometry. The pyramidal elements were present in the regions close to the wall as they are more capable of preserving the curvature of the wall boundary. These meshes were later used to establish the grid-convergence results.

A single mesh was created for the multiple-bend geometry. The mesh consisted of about 3 million tetrahedral and about 700,000 pyramidal elements. 
A steady state, laminar, and pressure based solver was chosen in the solver settings of the software. The pressure velocity coupling was resolved using the SIMPLE scheme. The best suitable spatial discretization scheme for the equations was not immediately apparent. At least three different choices had to be tested. For this purpose the UPWIN, QUICK, and 3rd order MUSCL schemes were selected as the premier options. The upwind scheme is computationally-light and hence picked as one of the choices. The QUICK scheme is second order and was expected to render more accurate results compared to UPWIND; and MUSCL was picked as the last choice as it is accurate to the third order. The results obtained from utilizing these three different schemes will be discussed in the subsequent section.

The convergence criterion for the setup was fixed by setting the residual value with a cap limit of about 10e-6. The coarse mesh took about 300 iterations to converge, and was closely followed by the medium mesh which took about 600 iteration, lastly followed by the fine mesh which took about 1100 iterations. These numbers were fairly constant over the varying spatial-discretization schemes. 


\section{Chapter 3: $\quad$ STAGGERED HERRINGBONE GEOMETRY RESULTS}

\subsection{Staggered Herringbone Geometry}

First the results pertaining to the Staggered Herringbone Geometry are presented and discussed.

\subsection{Grid Convergence}

Grid Independance

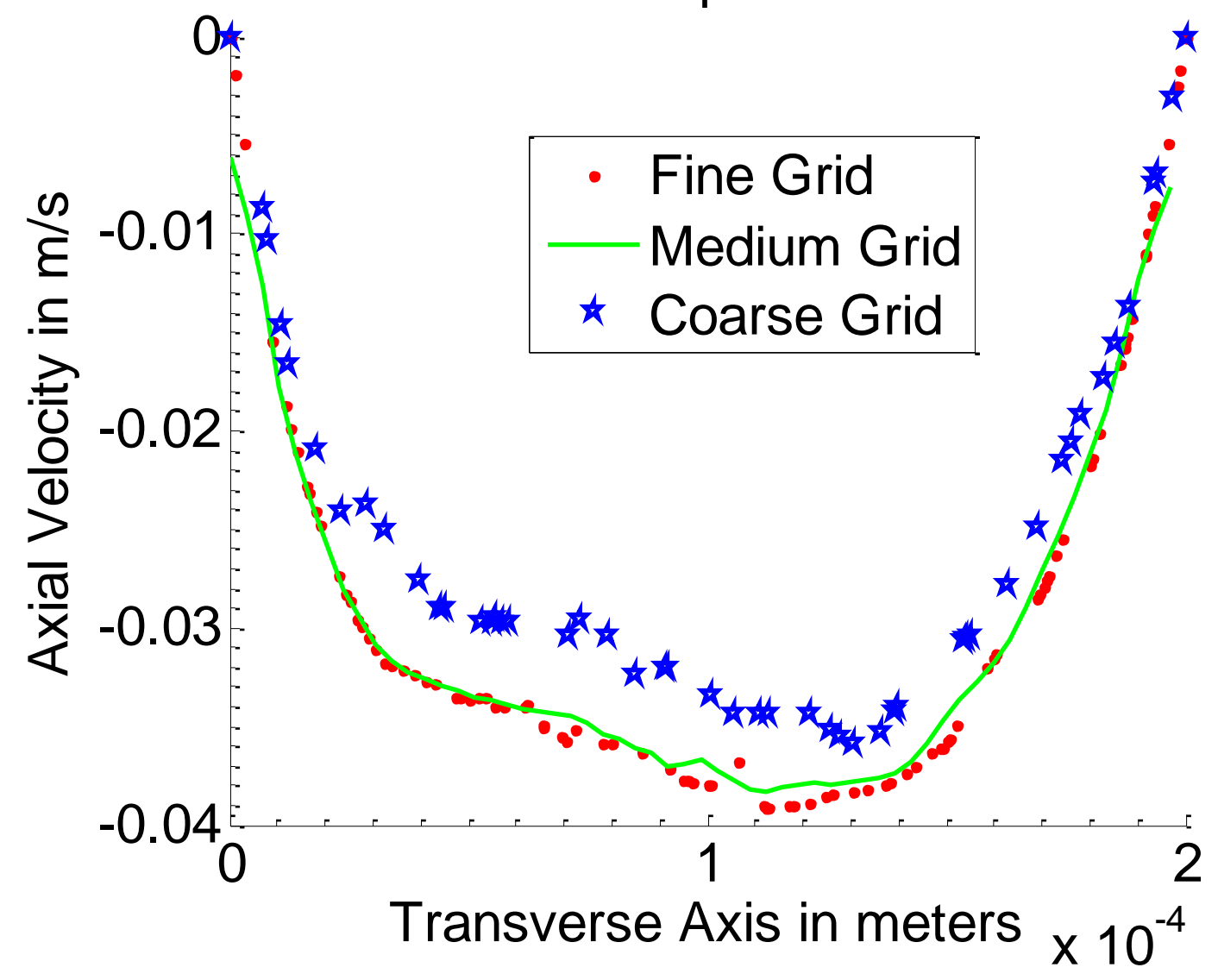

Figure 3-1: HGB Channel Grid Convergence 
Generating an adequate mesh was a prerequisite that had to be met prior to obtaining results for the subsequent analysis. The geometry utilized for this exercise was the herringbone configuration. This choice was reasonable given the fact that the herringbone configuration had been extensively studied in preexisting literature ${ }^{[5]}$. Accordingly, three meshes were created. Details regarding the meshes were as described above. All the three meshes were utilized to resolve the solution data employing the MUSCL scheme. The axial velocity plots were generated at the outlet of the channel for each of the three mesh configurations; see Figure 3-1. The data were extracted at the outlet from a transverse running guide about half-way deep into the channel.

Figure 3-1 shows that the curves tend to overlap as the mesh size increases. As a result, the solution on the finest grid is assumed to be converged with respect to the grid size. Consequently, the fine mesh was utilized for all subsequent computations performed thereafter in the study.

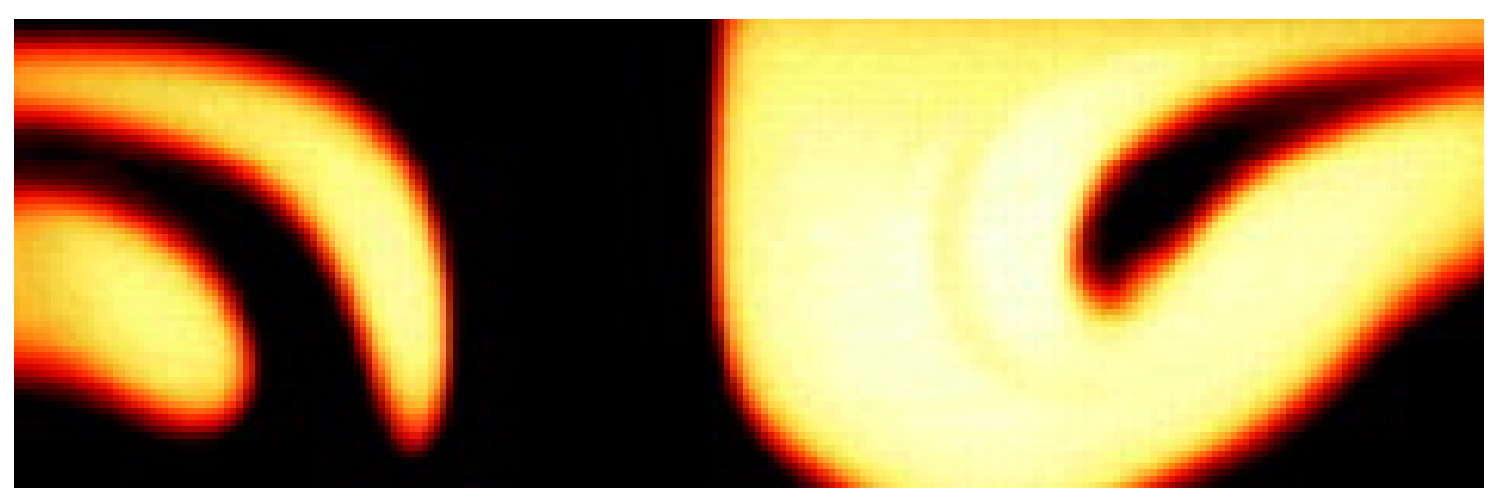

Figure 3-2: Image profile from literature ${ }^{[15]}$ 


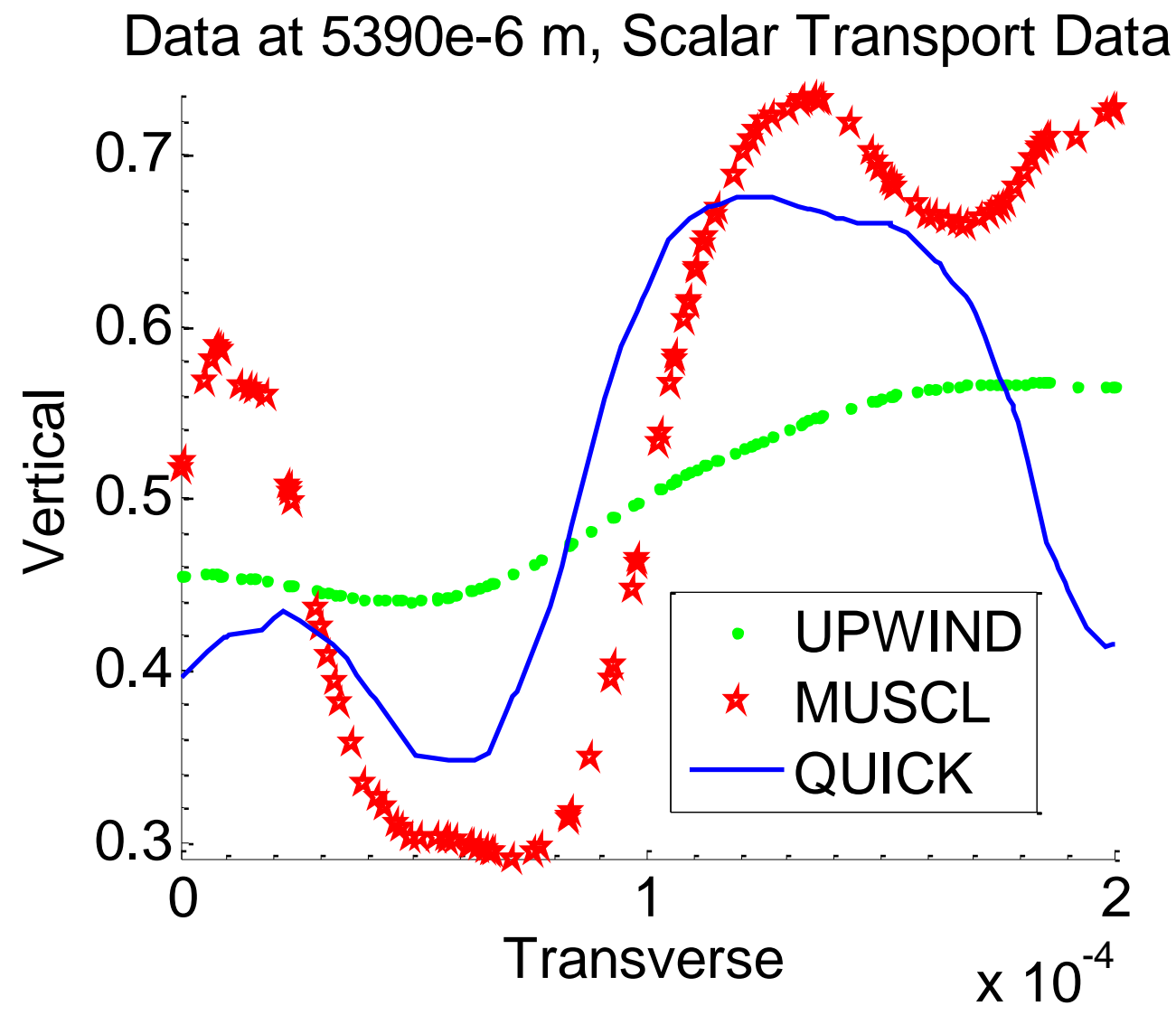

Figure 3-3: HGB geometry scheme convergence

\subsection{Comparison of Different Numerical Schemes}

As mentioned in the previous section, the UPWIND ${ }^{[16]}$, QUICK ${ }^{[16]}$, and MUSCL ${ }^{[16]}$ schemes were selected as possible alternatives to resolve the problem. Prior to discussing the results, it may be mentioned that the MUSCL scheme belongs to the class of discretization scheme classified as total variation diminishing (TVD) [17]. TVD schemes are generally known for having minimal numerical errors. In addition, the TVD schemes are known to generate results with reduced numerical-overshoots (errors) compared to the QUICK scheme ${ }^{[18]}$.

The three methods were resolved employing the fine mesh. Mass-fraction data were extracted at the half-way point along the axial length of the channel from a horizontal guide positioned half-way deep into the channel. Also available were qualitative data 
for the experiment from source [5] at the exact location. The experimental data is a snapshot of the fluids undergoing mixing at the half-way point along the length of the channel.

The results obtained using different discretization schemes are compared with the experimental snapshot to identify the scheme that renders data which can be translated to most closely match the information presented in the snapshot. Ideally, using qualitative information to validate a quantitative method is not the most desirable approach. Nevertheless, owing to the lack of available quantitative experimental data this exercise was deemed appropriate. Figure 3-2 represents the cross-section obtained from the publication ${ }^{[5]}$. The cross-section was extracted at about 5390e- $6 \mathrm{~m}$ along the channel measured from the inlet. From the cross-section snapshot shown in Figure 3-2, it may be observed that the fluids in the channel remain relatively unmixed. As a result, the scheme that renders mass-fraction data representing the unmixed fluids should be considered the most accurate for these simulations. Figure 3-3 represents mass fraction data obtained from solving the scalar transport equation employing the UPWIND, QUICK, and MUSCL schemes. The curve obtained from the UPWIND is relatively flat with mass-fraction values in the vicinity of 0.5 which indicates close to thorough mixing due to numerical diffusion. For the other two curves, it appears that their peaks tend to fluctuate along the transverse axis, see Figure 3-3. This observation is consistent with the snapshot as either fluid fold into the other along the transverse axis; see Figure 3-2. However, the peaks observed in the curve representing the MUSCL scheme (Red in Figure 3-3 seems to be more prominent compared to the subdued peaks characterizing the curve representing the QUICK scheme (curve in blue). A more prominent peak is reminiscent of an unmixed fluid and as a result the MUSCL scheme is more representative of the snapshot. Consequently, all subsequent analyses considered in the study were computed utilizing the results obtained from the MUSCL scheme. 


\subsection{Results}

The next set of data discussed in the section is the most significant for the purpose of the current study.

Prior to discussing the results, it may be noted here that each of the figures presented in this section have a description included at their top. This description is included to give the reader a brief overview of few key observations regarding the figure.

Figure 3-4(ii) shows point-cloud data generated utilizing streamtraces. Each figure is specific to the downstream location from which data were extracted; 15400 tracers were evenly distributed along the cross-section of either inlet; see Figure 3-4 (i). The points were colored by their location at the inlet. The blue points represent the fluid on the left inlet while green represents the fluid on the right. Despite introducing 15400 tracers not all of them could be tracked the entire length of the channel. This can be inferred by considering the point-cloud data in the downstream plots; for instance, comparing Figure 3-4 (i) and Figure 3-8 (i), it can be seen that there are regions of empty voids close to the boundary in Figure 3-8 (i). These voids exist because there were no streamtraces identified in this region. This observation of missing points is not limited to the current study and has been reported in earlier studies involving similar analysis [19]. There are two possible reasons for termination of streamtraces within the computational domain and the absence of the point-cloud data in certain regions. The first reason is a shortcoming of the post-processing tool utilized to generate the data. Interested readers are referred to Teccplot's reference manual that presents an exhaustive discussion on this topic. The second reason is that the points tend to overlap and remain hidden one behind the other. This happens when the fluid in the channel undergoes stretching and folding. The stretching and folding is a consequence of adding baffles to the geometry. It may be recalled that the purpose of adding baffles to the geometry was to induce chaotic mixing in an otherwise laminar flow configuration. The second set of cross-sections shown in the figure [Figure 3-4 (ii), Figure 3-3(ii), Figure 3-6 (ii), Figure 3-7(ii), and Figure 3-8 
(ii)] represent the point-cloud data after they have been post processed and grouped into bins as discussed earlier. For the sake of convenience these plots will be referred to as the gridded-data. The color intensity on each grid characterizes a mass-fraction like datum. This data enabled translation of qualitative information into quantitative information. The third set of cross-sections shown in the figure [Figure 3-4 (iii), Figure 3-5 (iii), Figure 3-6 (iii), Figure 3-7 (iii), Figure 3-8 (iii)] represent qualitative data obtained from [5] for liquid-2 on the right. These snapshots report some very interesting information.

The fluids tend to fold into each other progressively in the downstream direction. For instance, the cross-section at the outlet has the most number of such folds; see Figure 3-8 (iii). This observation does not necessarily indicate mixing. This is because the fluids can still maintain their individual homogeneity in the folded state. In fact the folding pattern essentially stretches the fluid; and stretching increases the contact surface area between the fluids. Progressively increasing the contact surface area should ultimately result in mixing. As a result, it can be concluded that the fluids remain unmixed at the outlet.

The fourth column in the figure reports profile plots of the mixing data; see [Figure 3-4(iv), Figure 3-5(iv), Figure 3-6(iv), Figure 3-7(iv), Figure 3-8(iv)]. The red curve represents mass-fraction data obtained from solving the scalar transport equation; refer to Eq. (1). The blue curve represents quantitative data extracted from the scattercloud data via Eq. (6). The plots [Figure 3-4(iv), Figure 3-5(iv), Figure 3-6(iv), Figure 3-7(iv), Figure 3-8(iv)] offer the most significant information with regards to the purpose of this current study. It has already been established that the fluids remain unmixed at the outlet. It should be noted that a mass fraction (or a volume fraction in this case) value of 1.0 or zero indicates no-mixing and 0.5 indicates complete mixing. If the fluids remain unmixed at the outlet, they should be unmixed in the upstream cross-sections as well. The blue curves in the plots have prominent fluctuating peaks across the transverse axis. This pattern is consistent with regards to the presence of the folds. For instance any two neighboring folds in the snapshot are occupied by 
either fluid; see Figure 3-6 (iii). Translating this information into a profile will characterize a curve with fluctuating peaks along the transverse axis; see Figure 3-6 (iv). As a result it is reasonable to assume that the quantified data using the new method are accurate.

The effect of numerical diffusion can clearly be seen in the profile of the red curves in the downstream direction. For instance, the red curve at the outlet indicates massfraction values close to half; see Figure 3-8 (iv). This value of 0.5 represents a homogenized mixture and is clearly in contradiction to the pattern observed in the snapshot; see Figure 3-8 (iii). The peaks of the red curves remain subdued in all the plots compared to the peaks of the blue curve. This information can be readily seen in the plot at the outlet. Notice that the blue curve has peak values fluctuating between 1.0 and 0.0, on the other hand the red curve hovers around 0.5; see Figure 3-8 (iv). This finding was expected as the numerical diffusion associated with the scalar transport method is much stronger compared to the streamtrace method. 
Figure (i) represents the point-cloud data at the inlet. There are 15400 green and 15400 blue points at the inlet. Figure (ii) represents the scatter-cloud data having been translated into qualitative mixing data (gridded data). It may be noted here that the information is scattered across a 20x30 grid; figure (iii) represents qualitative experimental results obtained from [5]; and figure (iv) represents a profile of the data obtained from figure (ii). It may be noted that all the profile were extracted along a horizontal guide about half-way high $(y=38.5 e-6 \mathrm{~m})$ along the vertical axis.

(i)

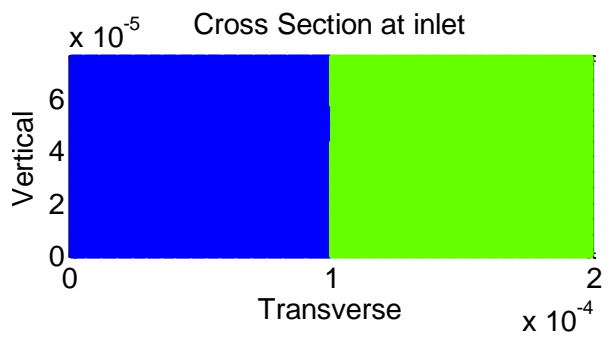

(iv)

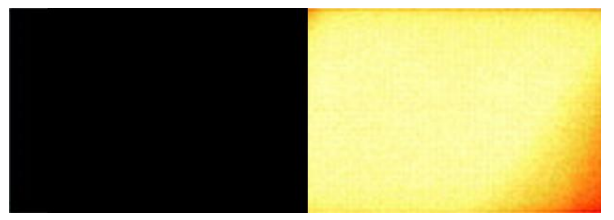

(iv)
Data extracted at Inlet $(0 \mathrm{~mm})$

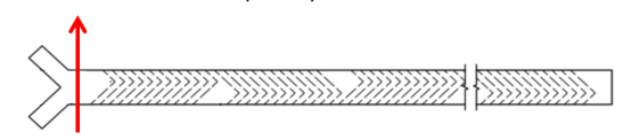

(ii)

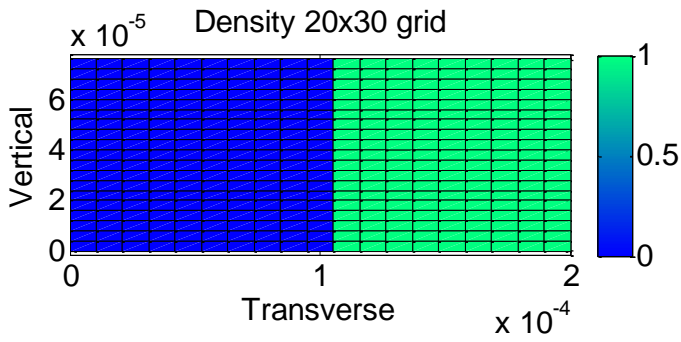

(vi)

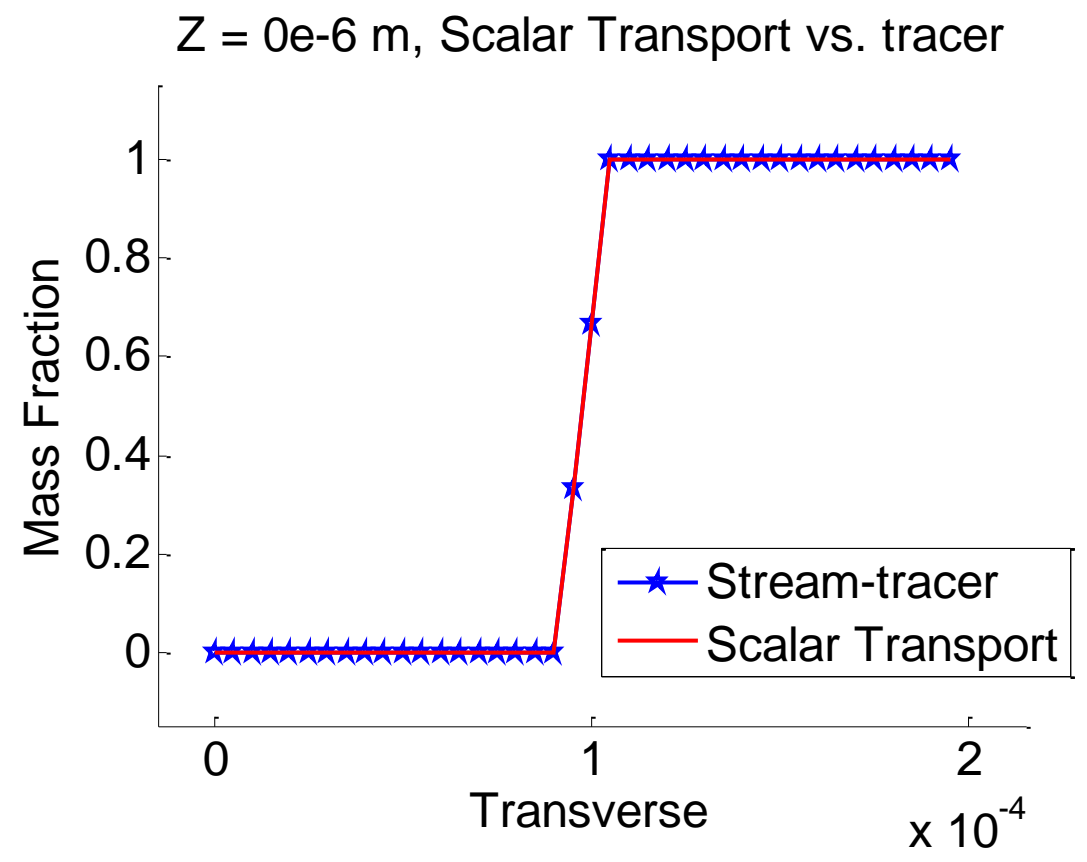

Figure 3-4: Staggered Herringbone geometry data at $0 \mathrm{~mm}$. 
Figures (i) through (v) represent plots post-processed from data extracted at 2090e-6 m downstream of the inlet. Figure (i) represents the point-cloud data; there are 12818 green and 11393 blue points. Figure (ii) represents the gridded data; figure (iii) represents qualitative experimental results obtained from [5]. Figure (iv) represents a profile of the data obtained from (ii).

(i)

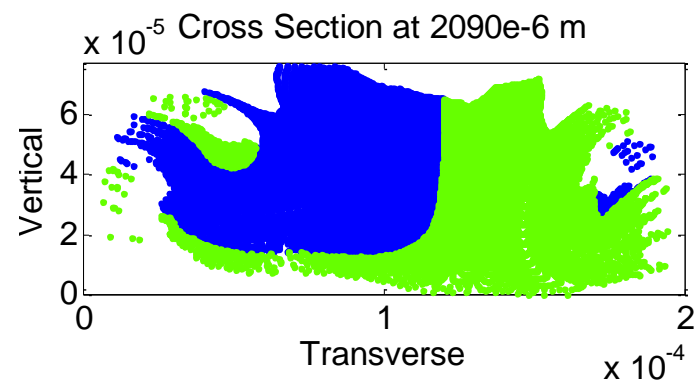

(iii)

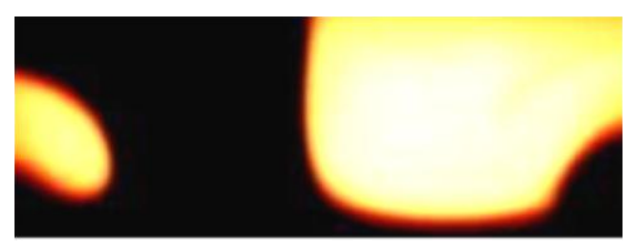

(ii)

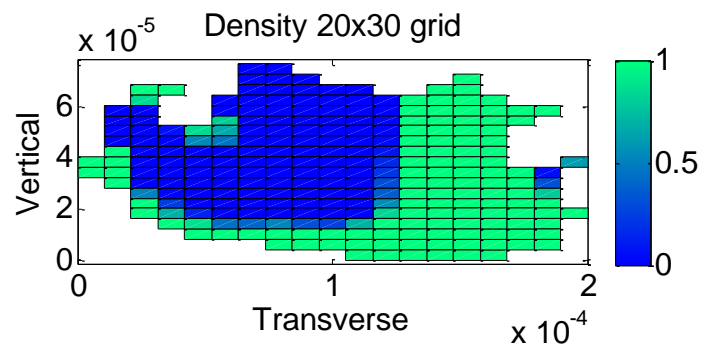

(v)

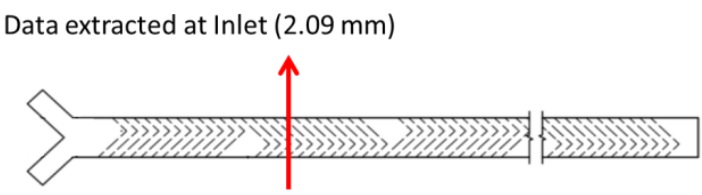

(iv)

\section{$\mathrm{Z}=2090 \mathrm{e}-6 \mathrm{~m}$, Scalar Transport vs. tracer}

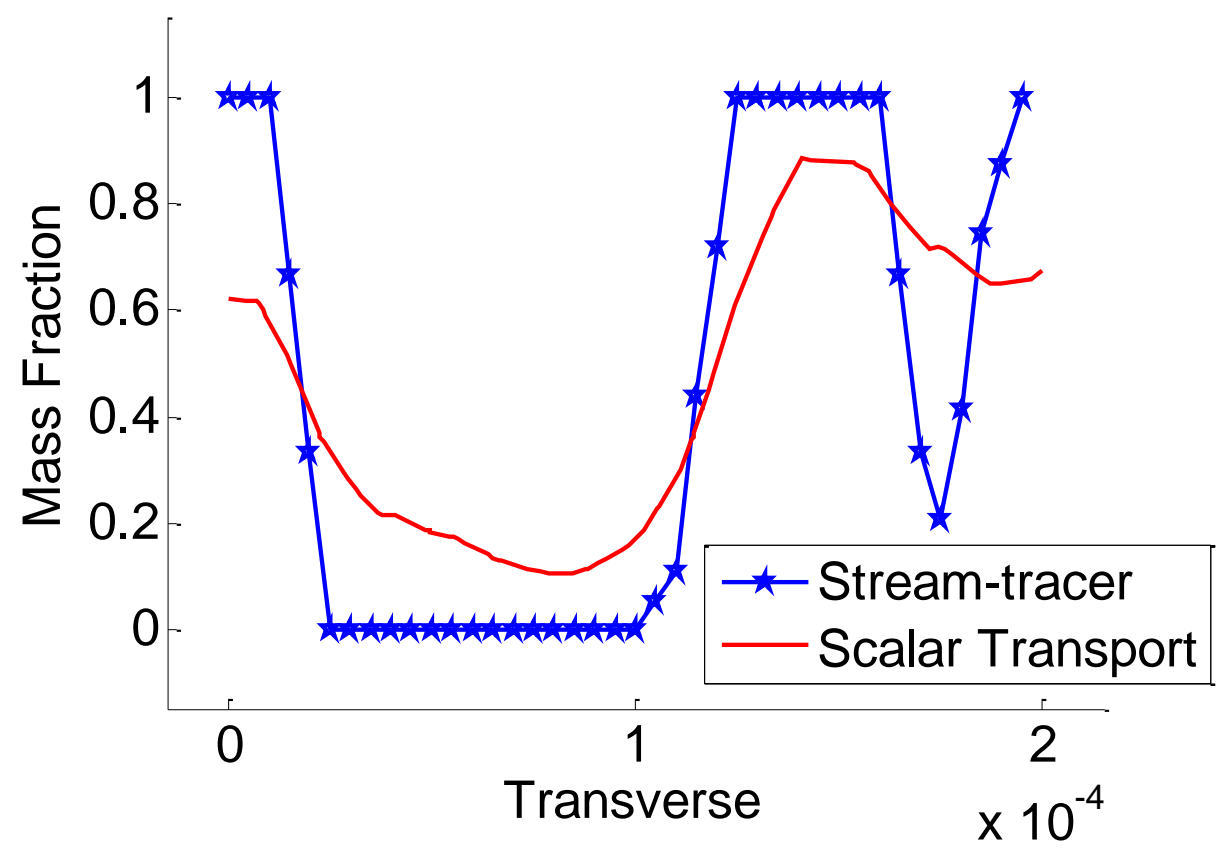

Figure 3-5: Staggered Herringbone geometry data at $2.09 \mathrm{~mm}$. 
Figures (i) through (v) represent plots post-processed from data extracted at 5390e-6 m downstream of the inlet. Figure (i) represents the point-cloud data; there are 8596 green and 7837 blue points. Figure (ii) represents the gridded data; figure (iii) represents qualitative experimental results obtained from [5]. Figure (iv) represents a profile of the data obtained from (ii).

(i)

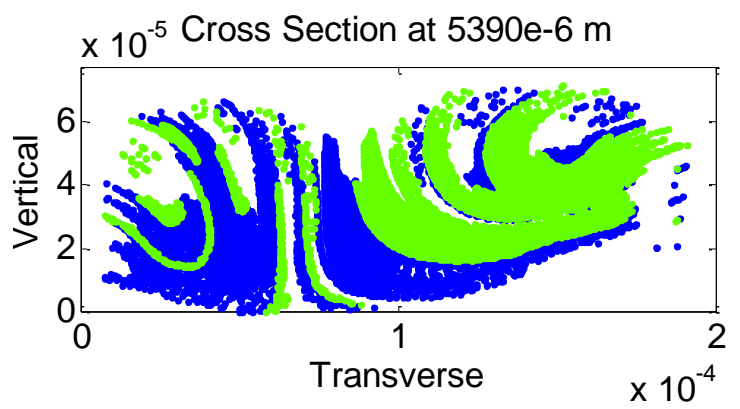

(iii)

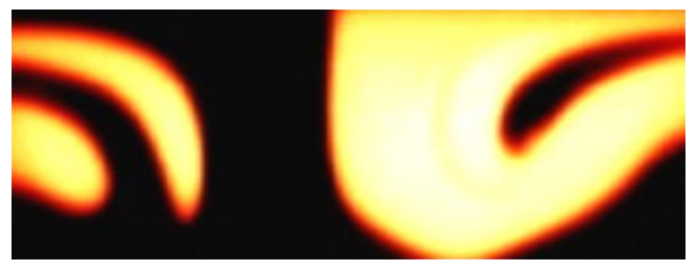

(ii)

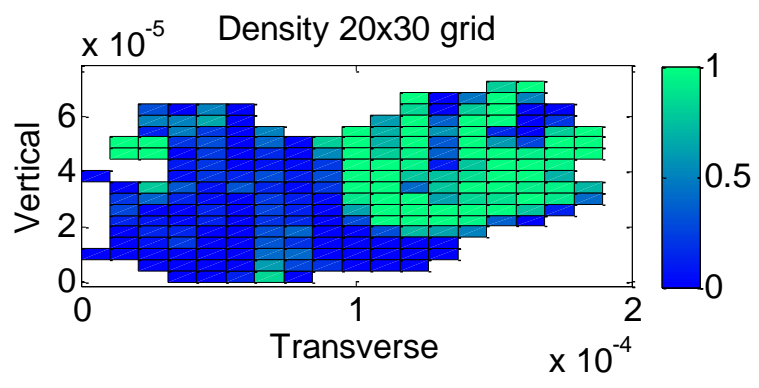

(v)

Data extracted at Inlet $(5.39 \mathrm{~mm})$

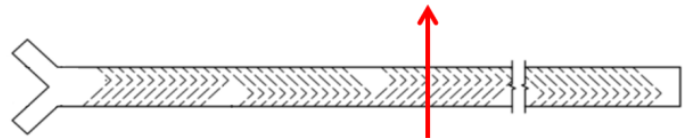

(iv)

\section{$\mathrm{Z}=5390 \mathrm{e}-6 \mathrm{~m}$, Scalar Transport vs. tracer}

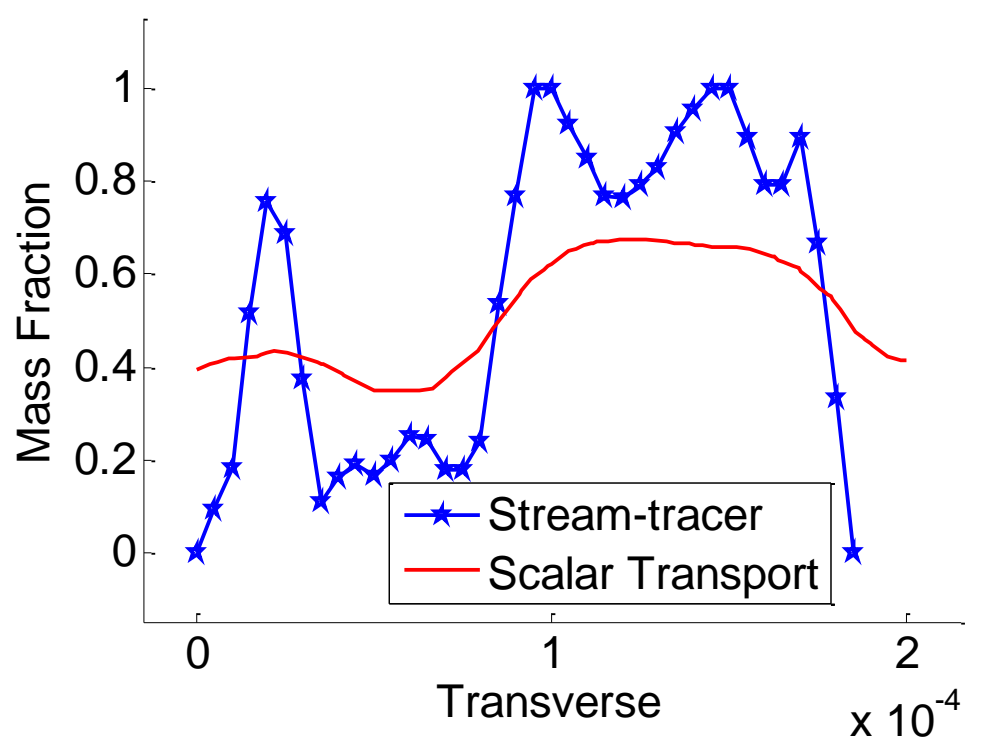

Figure 3-6: Staggered Herringbone geometry data at $5.39 \mathrm{~mm}$. 
Figures (i) through (v) represent plots post-processed from data extracted at 7590e-6 $\mathrm{m}$ downstream of the inlet. Figure (i) represents the point-cloud data; there are 6520 green and 5477 blue points. Figure (ii) represents the gridded data; figure (iii) represents qualitative experimental results obtained from [5]. Figure (iv) represents a profile of the data obtained from (ii).

(i)

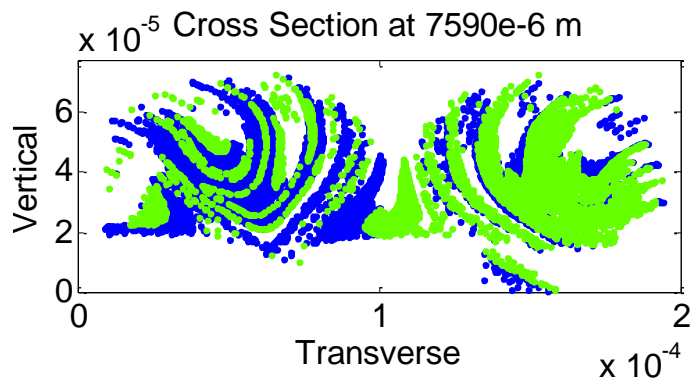

(iii)

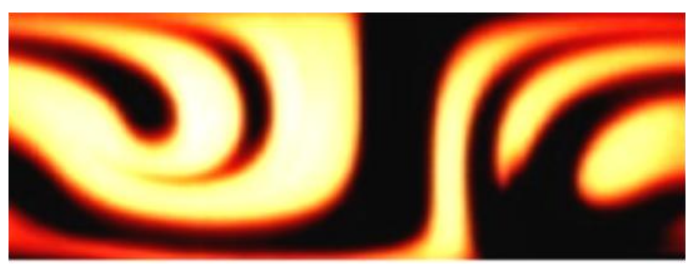

(ii)

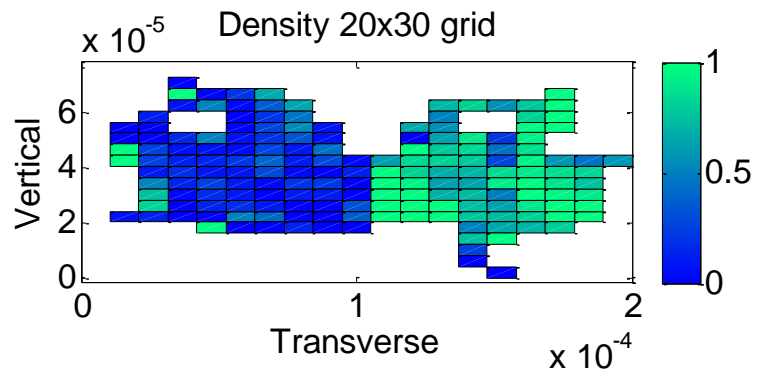

( v)

Data extracted at Inlet $(7.59 \mathrm{~mm})$

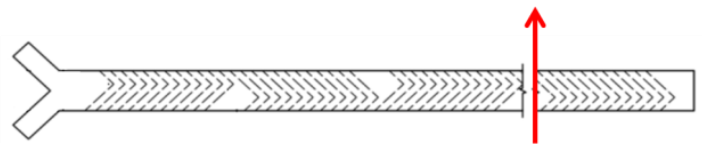

(iv)

$Z=7590 \mathrm{e}-6 \mathrm{~m}$, Scalar Transport vs. tracer

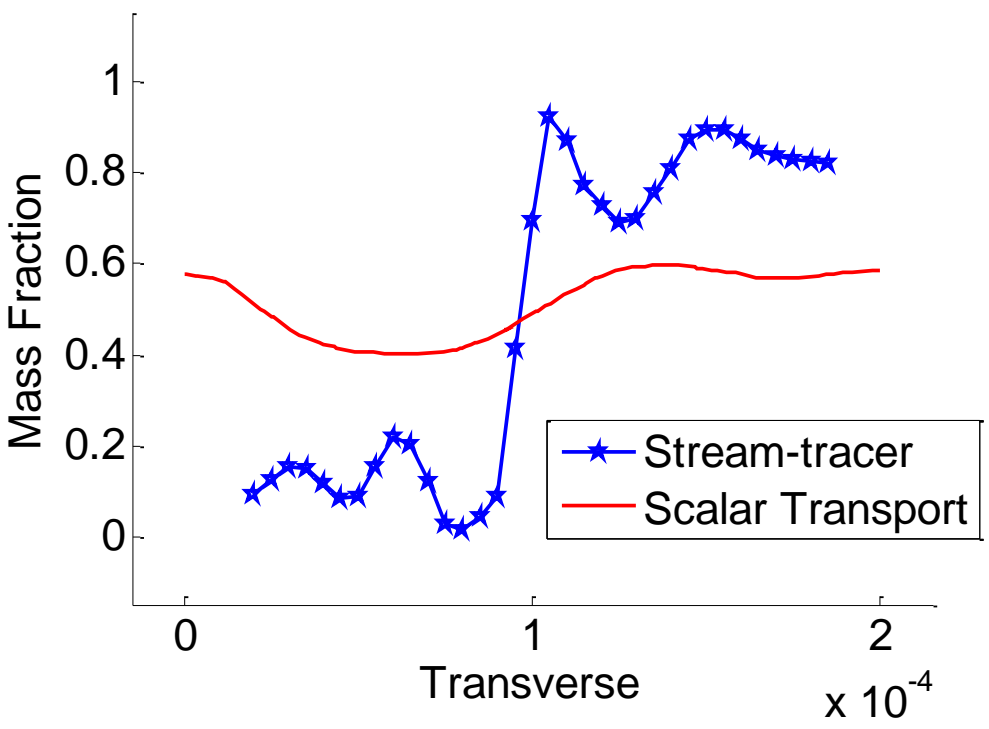

Figure 3-7: Staggered Herringbone geometry data at $7.59 \mathrm{~mm}$. 
Figures (i) through (v) represent plots post-processed from data extracted at 10890e-6 m downstream of the inlet. Figure (i) represents the point-cloud data; there are 3578 green and 3515 blue points. Figure (ii) represents the gridded data; figure (iii) represents qualitative experimental results obtained from [5]. Figure (iv) represents a profile of the data obtained from (ii).

(i)

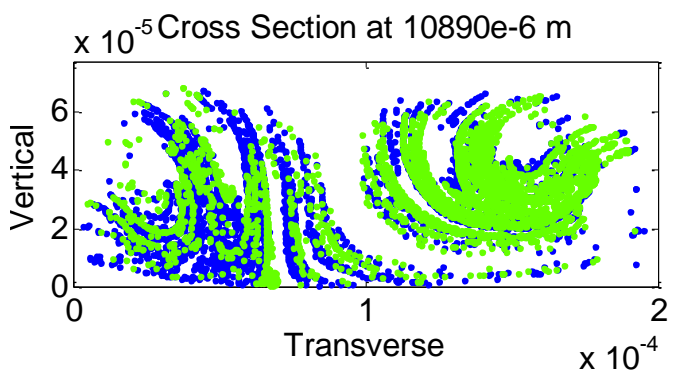

(iii)

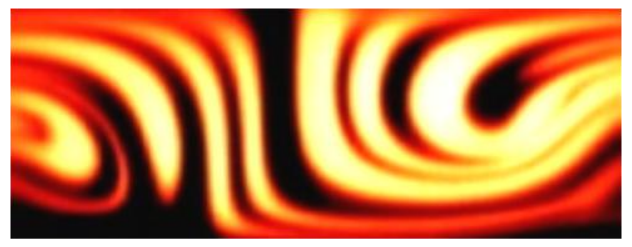

(ii)

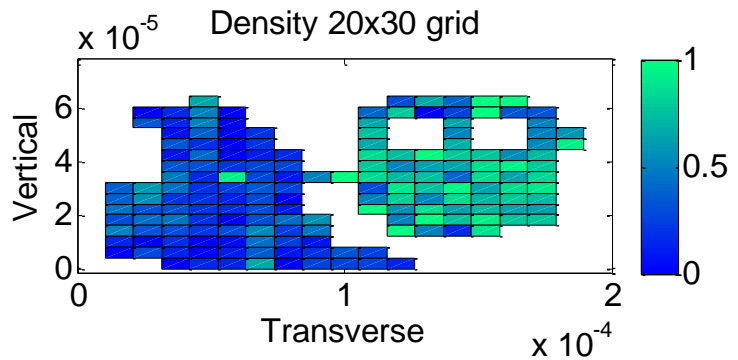

(v)

Data extracted at Inlet $(10.89 \mathrm{~mm})$

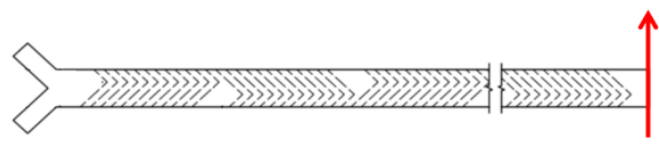

(iv)

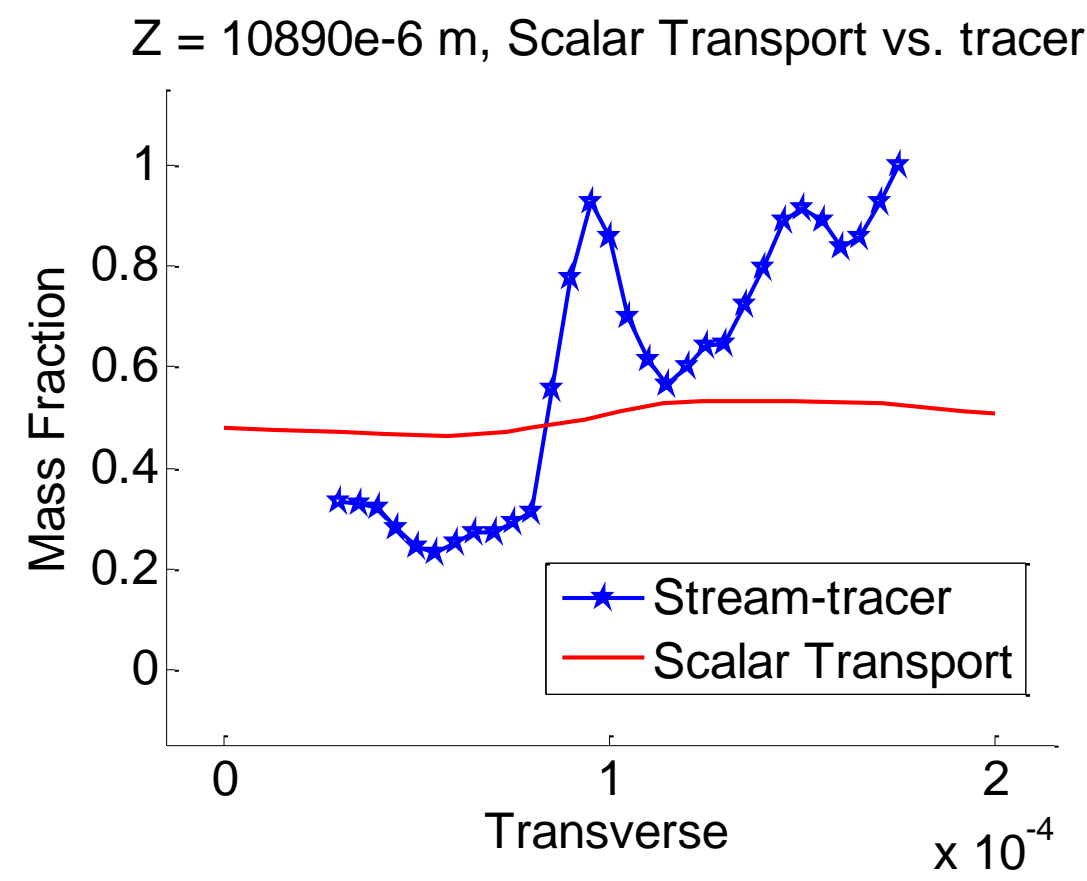

Figure 3-8: Staggered Herringbone geometry data at $10.89 \mathrm{~mm}$. 


\section{Chapter 4: MULTIPLE BEND GEOMETRY RESULTS}

The results pertaining to the multiple-bend geometry are presented and discussed in this chapter.

Prior to discussing the results, it may be noted here that each of the figures presented in this section have a description included at their top. This description is included to give the reader a brief overview of few key observations regarding the figure.

The multiple-bend geometry occupied about 8 times the volume occupied by the Herringbone geometry. If the same grid size as that of coarse mesh used earlier were to be employed for this geometry, the resulting mesh would have a staggering eight million elements. A mesh of this size is very challenging to build and also computationally heavy. However, the purpose of the current study remains to validate the quantification method and not exclusively analyze the new geometry. Thus a three million element sized mesh is used and will be sufficient for the study.

Furthermore, on the fairly coarse mesh of approximately three million nodes the simulations resulted in effectively no mixing between the two fluids. Given that most numerical solutions are dissipative with significant artificial diffusion on coarse mesh, it was not necessary to refine the mesh further. As such practice would not improve the results with respect to mixing intensity.

The MUSCL scheme was used to resolve the multiple-bend geometry since it did a very good job with the herringbone geometry. In addition there is only marginally available experimental data to validate these results.

\subsection{Results}

The next set of data discussed in the section is the most significant for the purpose of the current study. 
Figures (i) and (iii) represent the cross-section extracted at Bend 1 - see (v) for location of bend 1; figures (ii) and (iv) represent the cross-section extracted at Bend 2 - see (v) for location of bend 2; figures (i) and (ii) represent the streamtrace data; and figures (iii) and (iv) represent the mass-fraction data obtained from the solving scalartransport equation. Also, available in figure (v) is the color codes for the contours in figures (iii) and (iv).

(i)

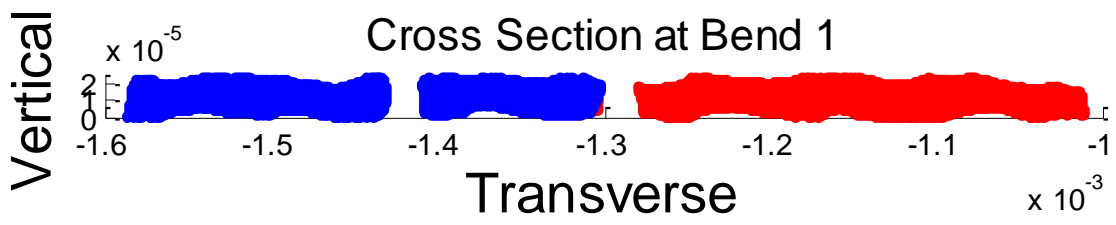

(ii)

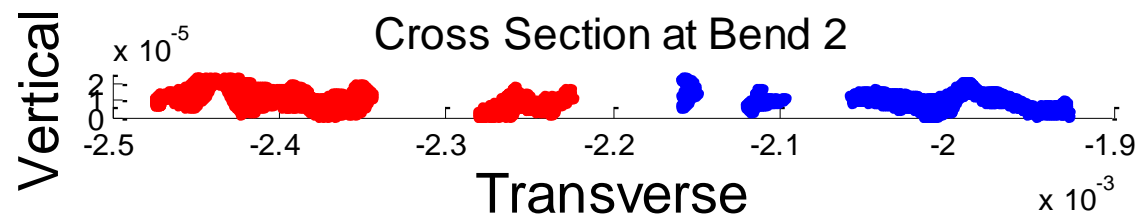

(iii)

(iv)

(v)

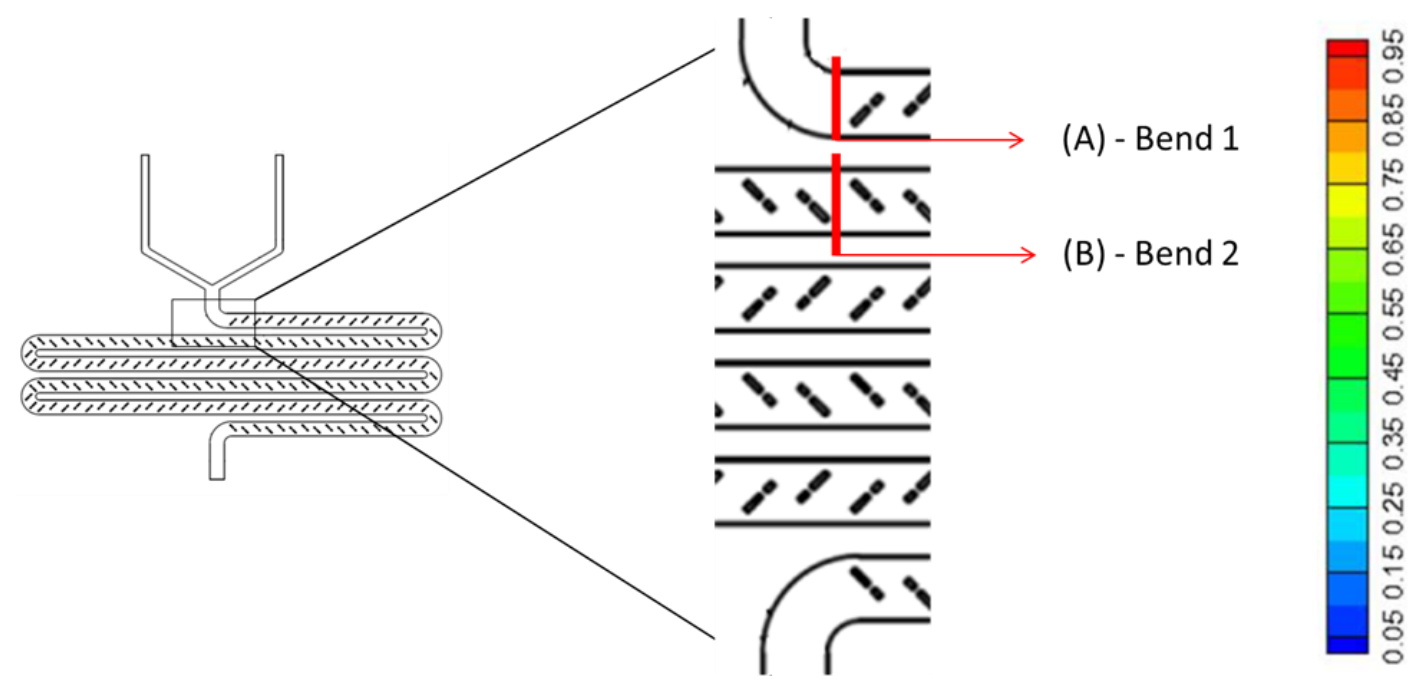

Figure 4-1: Multiple bend channel data at bends 1, and 2 
Figures (i) and (iii) represent the cross-section extracted at Bend 3 - see (v) for location of bend 3; figures (ii) and (iv) represent the cross-section extracted at Bend 4 - see (v) for location of bend 4; figures (i) and (ii) represent the streamtrace data; and figures (iii) and (iv) represent the mass-fraction data obtained from the solving scalartransport equation. Also, available in figure (v) is the color codes for the contours in figures (iii) and (iv).

(i)

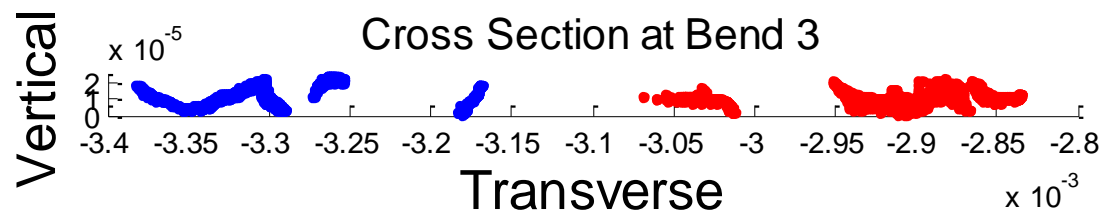

(ii)

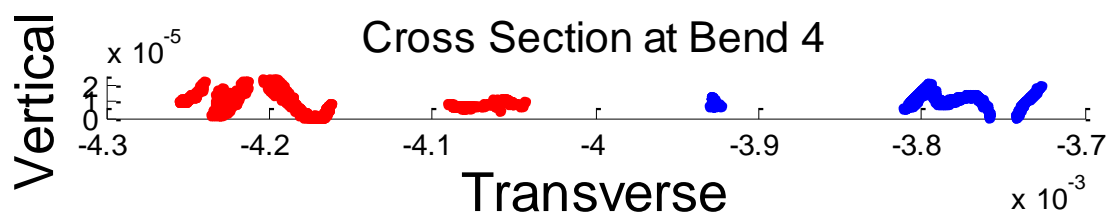

(iii)

(iv)

(v)

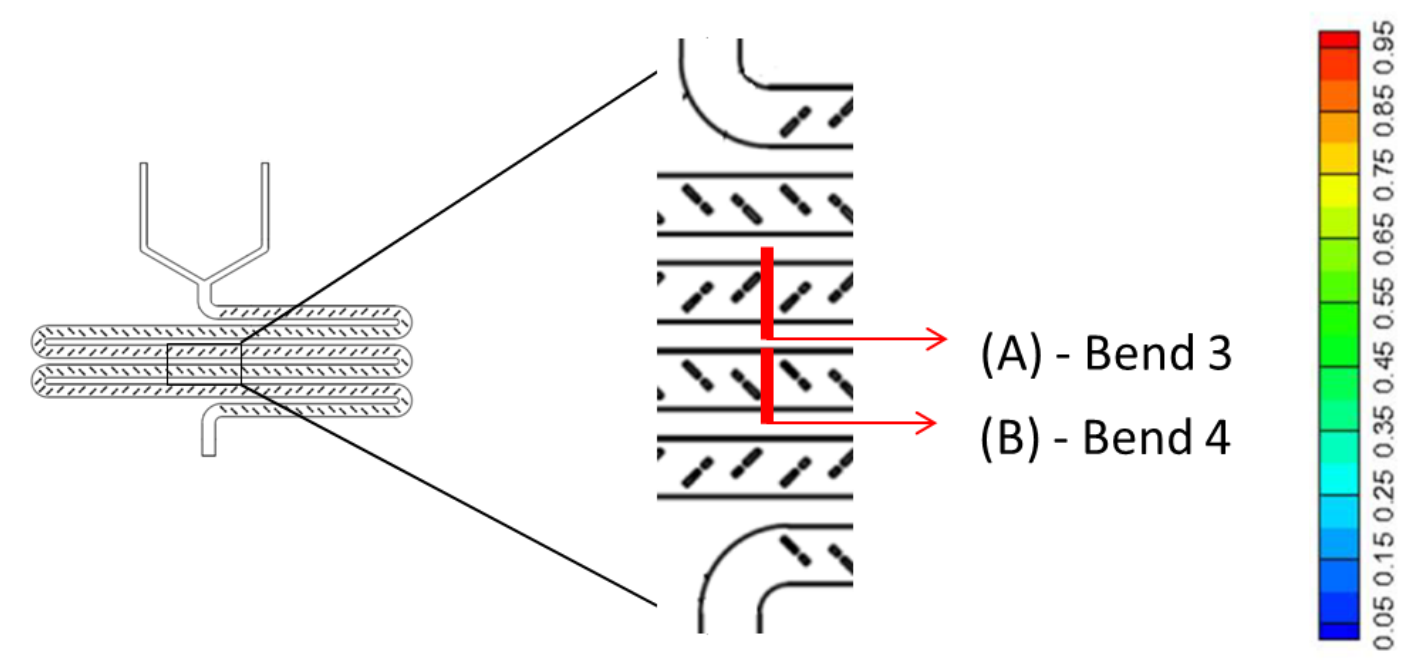

Figure 4-2: Multiple bend channel data at bends 3 , and 4 
Description: Figures (i) and (iii) represent the cross-section extracted at Bend 5 - see (v) for location of bend 5; figures (ii) and (iv) represent the cross-section extracted at Bend 6 - see (v) for location of bend 6; figures (i) and (ii) represent the streamtrace data; and figures (iii) and (iv) represent the mass-fraction data obtained from the solving scalar-transport equation. Also, available in figure (v) is the color codes for the contours in figures (iii) and (iv).

(i)

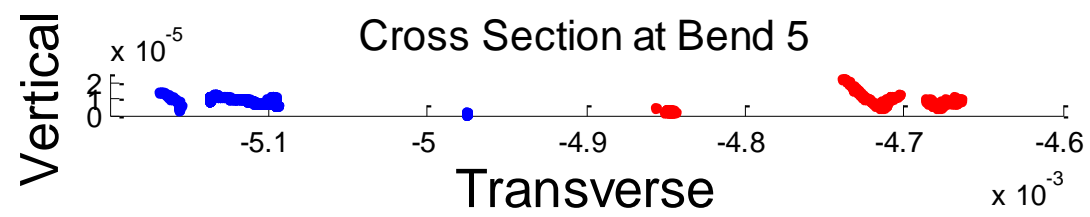

(ii)

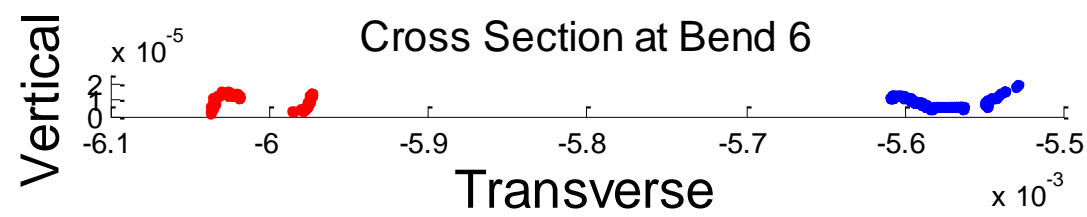

(iii)

(iv)

(v)

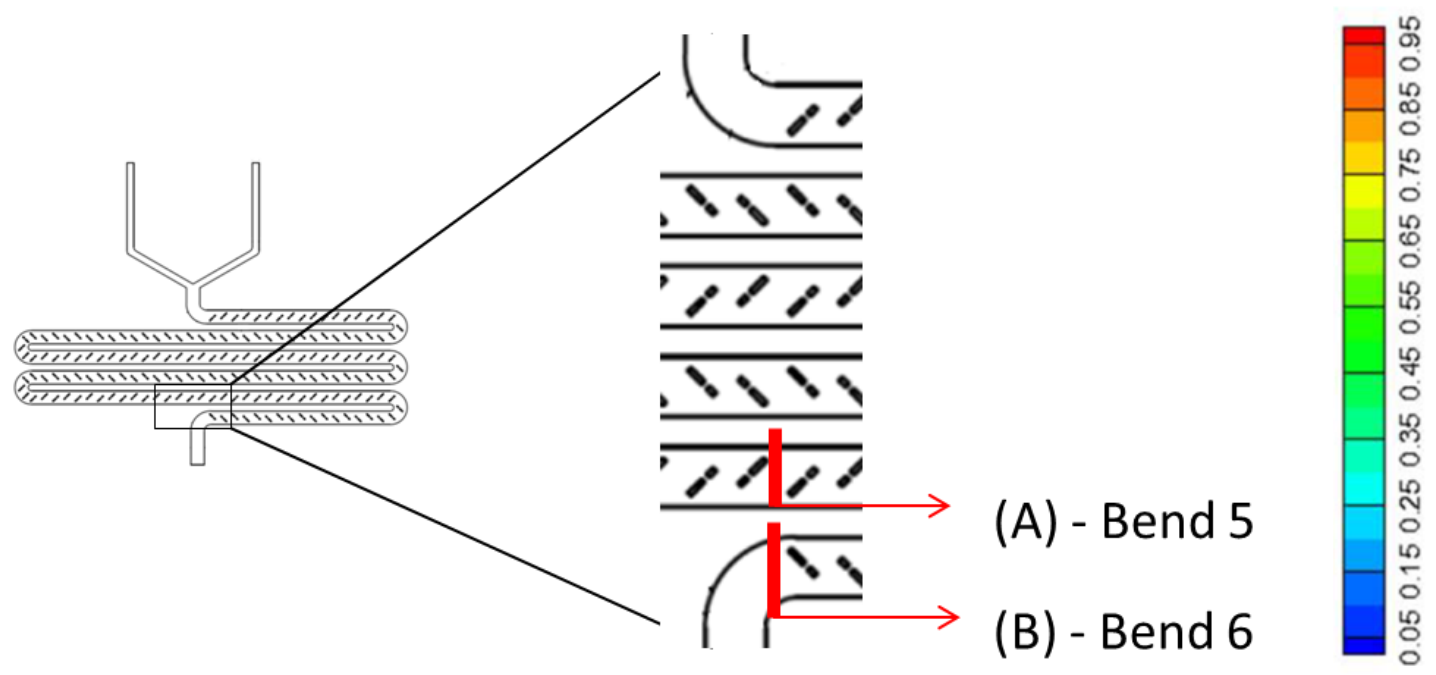

Figure 4-3: Multiple bend channel data at bends 5, and 6 
The multiple-bend geometry consists of six bends. Thus data was extracted along 6 cross-sections. The first set of cross-sections represents the scatter-cloud data obtained from streamtraces; see Figure 4-1(i and ii), Figure 4-2 (i and ii), and Figure 4-3(i and ii). About 4400 points were tracked at either inlet. Unlike the previous geometry, fewer streamtraces were utilized to generate data. This was a consequence of having to deal with a narrower cross-section; i.e. the ratio of the vertical depth to the transverse length was very small. Void regions missing scatter-data can be seen in the plots; see Figure 4-1 (i and ii). However, unlike the previous geometry, the location of the voids extends in the vertical direction throughout the depth of the channel. This was a consequence of walls being very close to each other. Walled baffles extend through the entire depth of the channel in the multiple bend geometry; see Figure 1-3. Also interesting to note are the positions of the streamtraces. The streamtraces representing either fluid maintain their position within the extent of their parent fluid; see Figure 4-1(i and ii), Figure 4-2 (i and ii), and Figure 4-3(i and ii). This means the fluids are not folded into each other. In the absence of folding the potential for mixing is very low. In this respect, the quantification method will not offer any new insight regarding mixing. Even if used, the quantified data would look similar along the entire length of the channel. Owing to the lack of comparable data, the influence of numerical diffusion on the scalar transport results is difficult to estimate. Figure 4-4 (i) and (ii) represents the profile of the mass-fraction values obtained from the scalar transport equation extracted at bend 1 - see Figure 4-1(iii) for the contour. The mass fraction profiles show sharper variations in the cross-section close to the inlet - see Figure 4-4 (i) - whereas in the downstream cross-sections the variation is somewhat smooth - Figure 4-4 (ii). This behavior could be attributed to the effect of numerical diffusion.

Figure 4-5 (i) represents the mass-fraction contour obtained from solving the scalar transport equation for the entire geometry. Figure 4-5 (ii) represents the qualitative snapshot obtained from [6]. Figure 4-5 (ii) indicates the fluids to have mixed consider outlet. However, Figure 4-5 (i) indicates the fluids to be unmixed. In the ongoing 
experiments on the micro-channel, however, adequate mixing is observed; see Figure 4-5(ii). This is a problem and will be addressed in the next chapter.

(i)

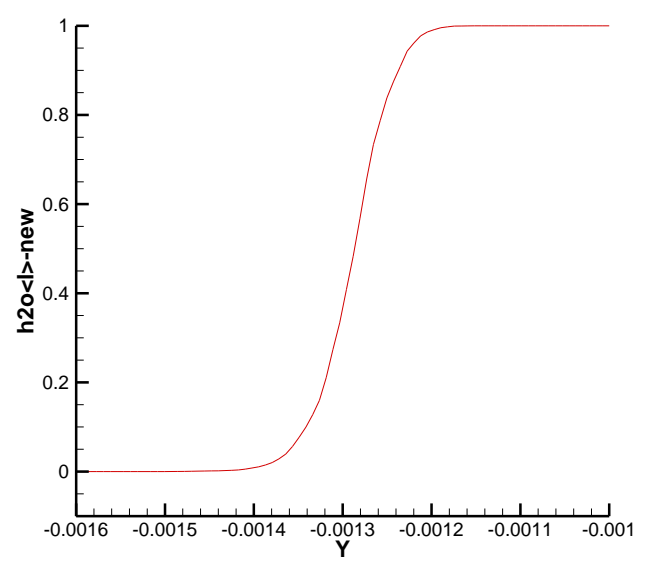

(ii)

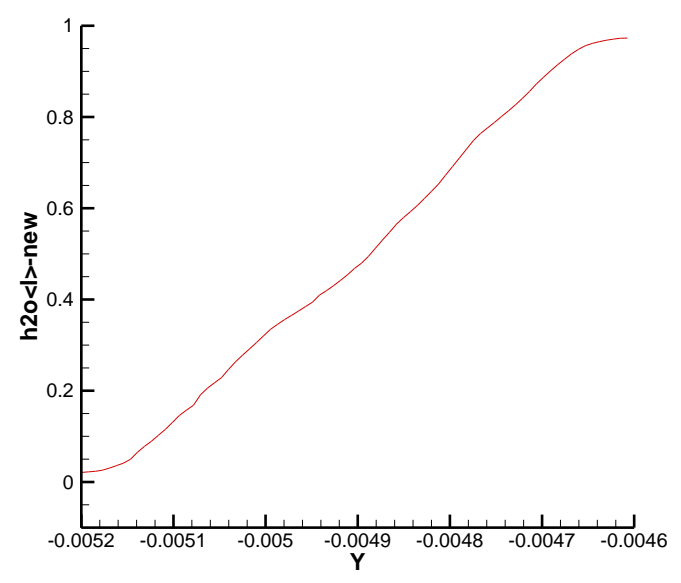

(iii)

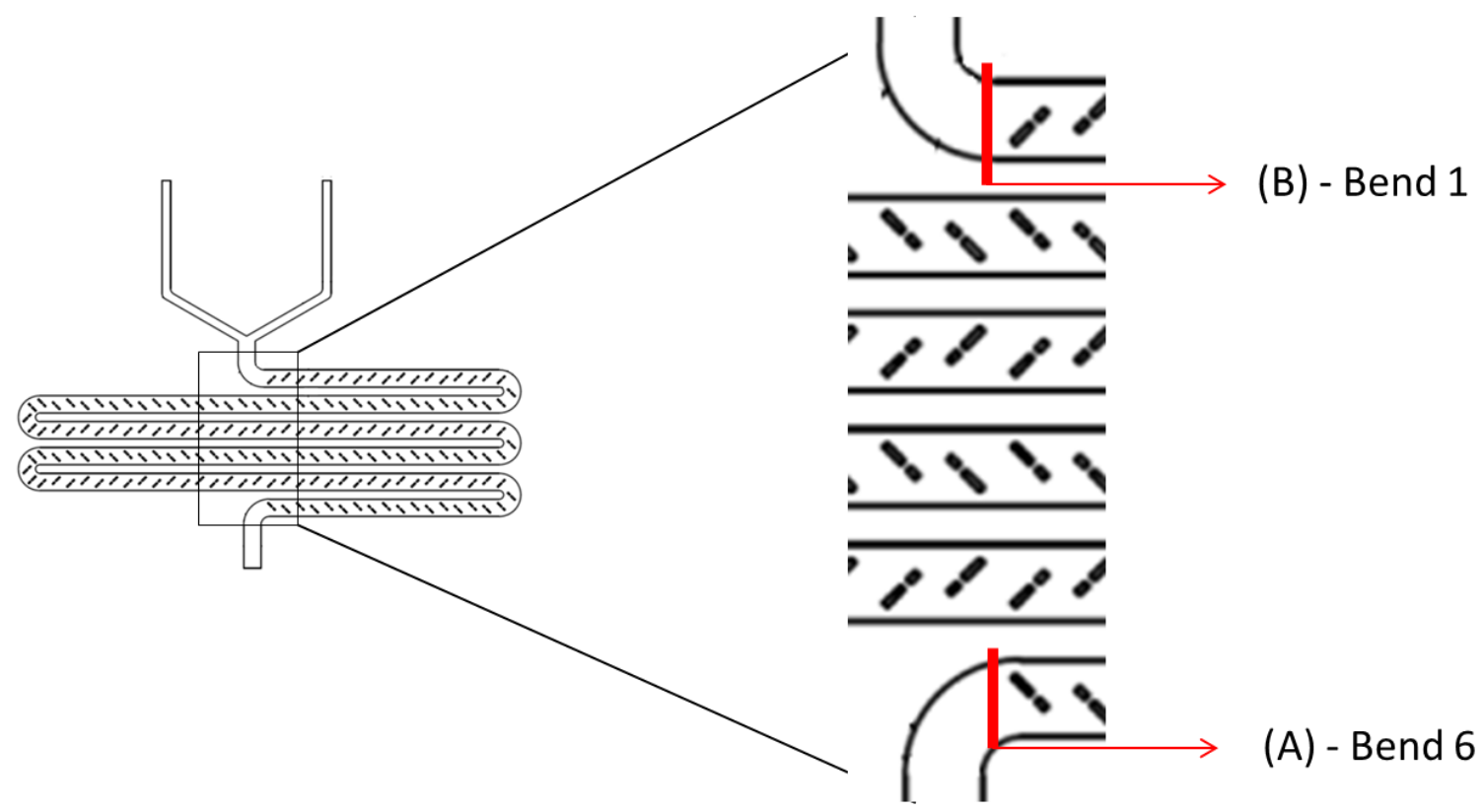

Figure 4-4: Multiple bend channel, mass-fraction profiles 
(i)

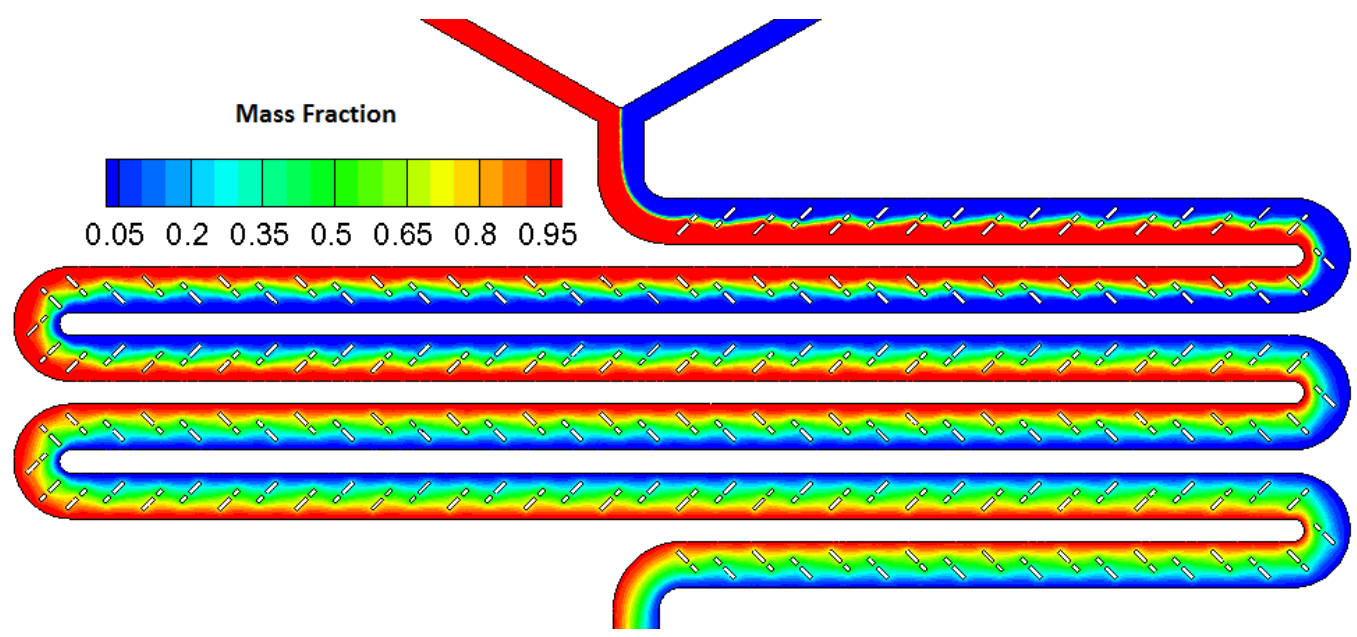

(ii)

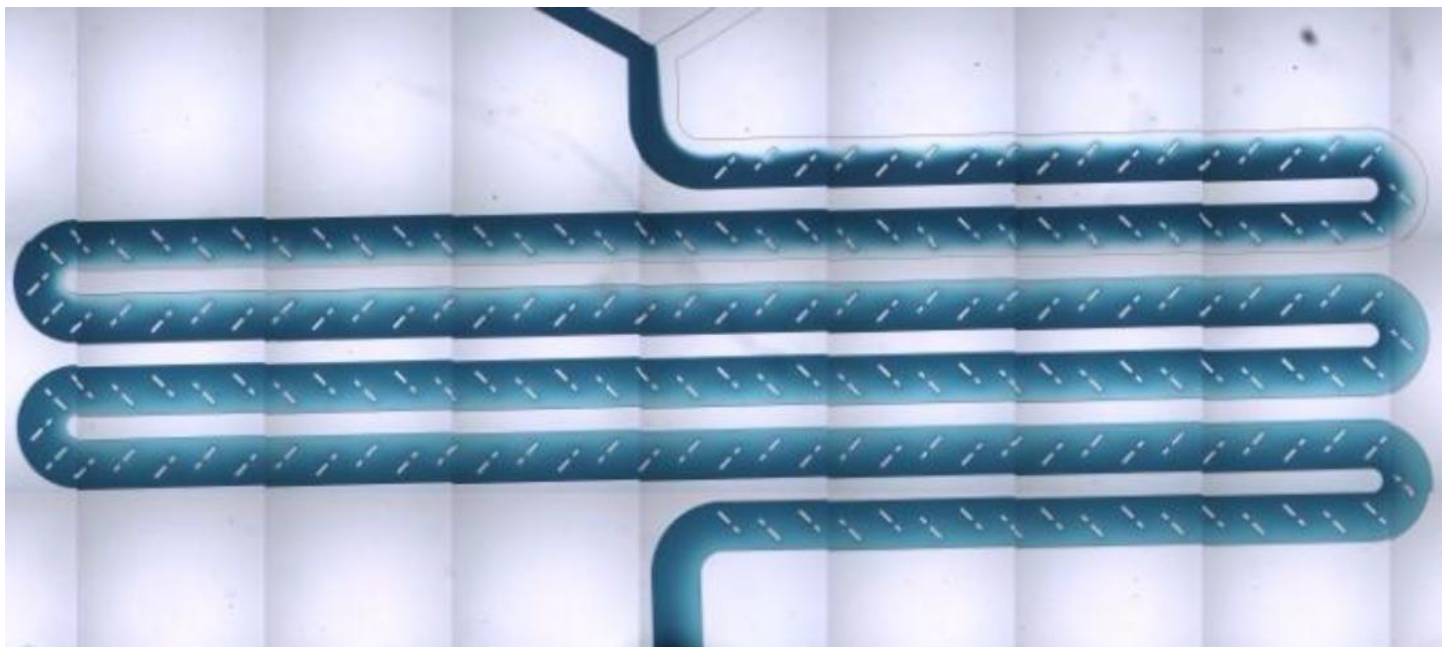

Figure 4-5: Multiple bend channel; Contour vs. Qualitative snapshot ${ }^{[6]}$ 


\section{Chapter 5: RESOLVING THE MULTIPLE BEND GEOMETRY}

\subsection{The Problem}

The mass-fraction data obtained from solving the scalar-transport (Eq. 1) was expected to suffer from numerical diffusion. It was anticipated that the extent of error would indicating complete mixing i.e. mass-fraction values lingering around 0.5 for the multiple-bend geometry. This was expected as a result of the data obtained for the HGB geometry in which the mass-fraction values indicated mixing while the streamtrace values did not - for instance see Figure 3-8 (iv).

However, as discussed in the previous section, the mass-fraction values despite suffering from numerical errors indicated unmixed fluids exiting the geometry; see Figure 4-4 (i), (ii). In complete contrast the qualitative results obtained from the experiment seemed to indicate mixed fluids exiting the system. Notice the blurring of the interface separating either fluid -the blue and white interface in Figure 5-5 (i).

One possible reason contributing to this observation could be associated with diffusivity values utilized in the Fluent-solver for the scalar transport equation.

While solving the scalar transport equation the diffusivity (of the fluids, one into the other) based on literature ${ }^{[15]}$ was set close to zero $\left(\sim 1 \mathrm{e}-15 \mathrm{~m}^{2} / \mathrm{s}\right)$. However, the actual experimental-run ${ }^{[6]}$ was carried out with a dye whose diffusivity was not known.

It was reasoned that if the diffusivity of the dye were known, then the scalar transport equation could be recalculated to derive better results. Consequently, it was decided that physical experiments would be carried out to determine the diffusivity of the dye. 


\subsection{Physical Experiment}

The first step was to calculate the diffusivity of the dye. A combination of numerical and experimental results was used to resolve this problem.

In order to successfully determine the diffusivity, a suitable experimental set-up was necessary. The apparatus included: a stable-uniform surface to support the setup, a transparent container to hold the fluids, a camera and tripod setup to capture images at regular intervals, a syringe to gently inject the dye, and finally, the dye ${ }^{[20]}$ itself.

The experiment involved introducing the dye into a beaker filled with water and allowing it to diffuse. A number of trial runs had to be performed prior to determining a working approach. Based on multiple trials, it was noted that the water in the beaker had to be full three/fourths to the brim $(\sim 4.75 \mathrm{~cm})$. This was necessary in order for the dye not to settle down (at the bottom) within the initial 15 minutes of the run -the dye is heavier than water and, the 15 minute time interval was sufficient to observe ideal behavior.

In addition, it was necessary only to consider diffusion of the dye in the radial direction. This consideration was in order to neglect the effects of gravity.

Once the apparatus was set up, the diluted-dye was gently injected $\left(\sim 1 \mathrm{e}-7 \mathrm{~m}^{3}\right)$ at the center of the beaker. Pictures were taken at regular intervals (see examples in Figure 5-1 and Figure 5-2). The radial spread of the dye was measured along the axes (two of them perpendicular to each other) parallel to the radial surface; notice the axes indicated by the red markers in Figure 5-1 and Figure 5-2. The spread of the dye was calculated from the center of the beaker. The measurements across either axis were averaged to generate a distance vs. time graph; the $0.5 \mathrm{~cm}$ wide dye patch shown in Figure 5-1 was obtained at an earlier instant compared to $2.4 \mathrm{~cm}$ wide patch shown in Figure 5-2. This approach was adopted based on recommendations from literature ${ }^{[21]}$.

Figure 5-3 describes the experimental results generated from averaging multiple runs under similar conditions using information from snapshots such as Figure 5-1and 
Figure 5-2. Five sets of individual runs were utilized to generate the experimental result. Each run lasted 15 minutes with snapshots (about 10) generated at regular intervals. The snapshots were obtained from a larger video file that recorded the entire experimental run. The first minute was usually neglected in order for the experiment to stabilize - this was a precautionary step to neglect the convective forces that might have been generated during the dye-injection (delivery) process.

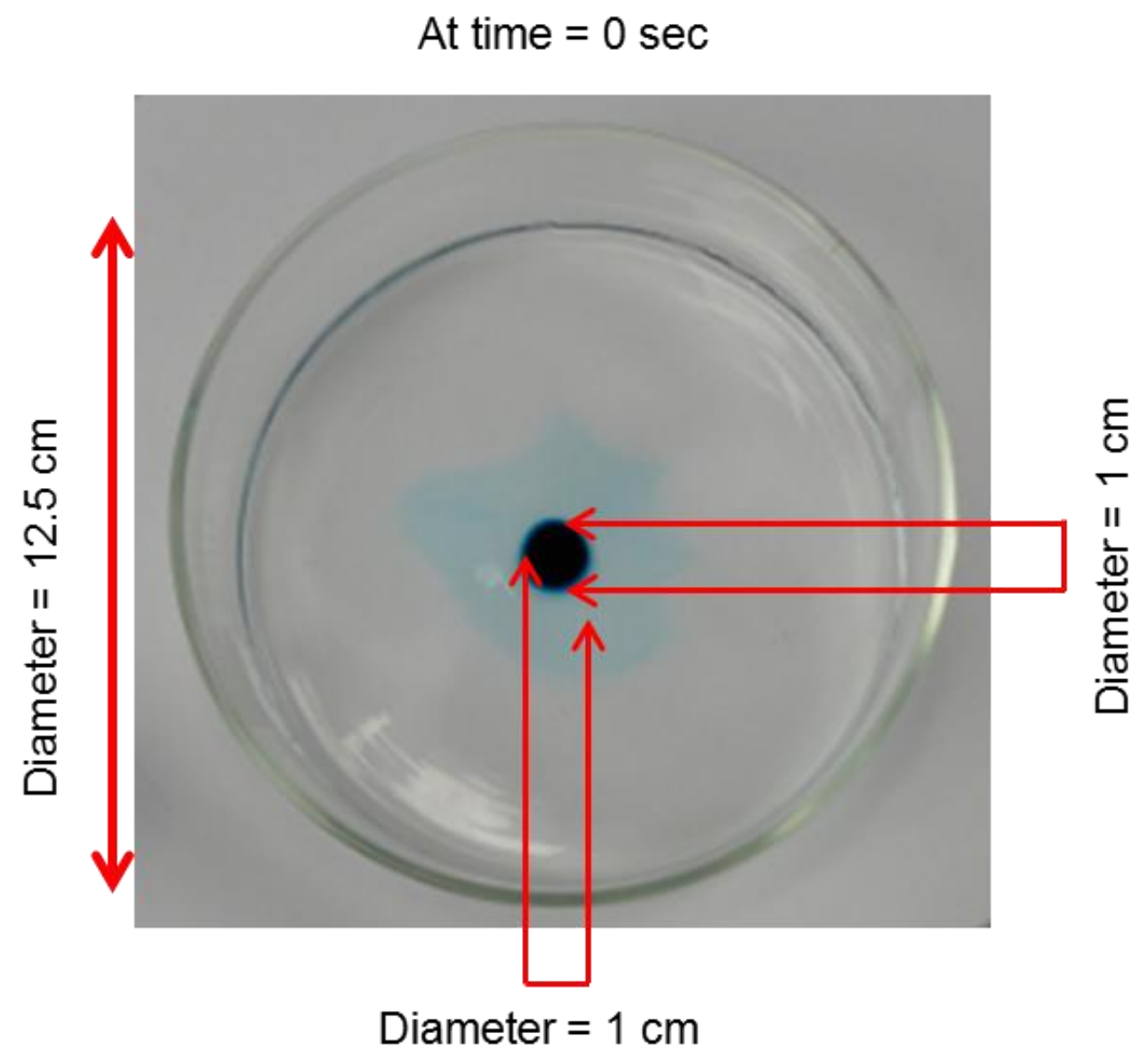

Spread of dye from center $=(1+1) / 4=0.5 \mathrm{~cm}$

\section{Figure 5-1: Initial Dye cloud}


Table 5-1: Density Values

\begin{tabular}{|c|c|}
\hline Type & Density \\
\hline Diluted Dye $^{*}$ & $1220 \mathrm{~kg} / \mathrm{m}^{\wedge} 3$ \\
\hline Dye & $1770 \mathrm{~kg} / \mathrm{m}^{\wedge} 3$ \\
\hline \multicolumn{2}{|c|}{ * The diluted dye utilizes a3:1 water to dye ratio; and the experiments were carried out using the diluted dye. } \\
\hline
\end{tabular}

At time $=2 \mathrm{~min}$

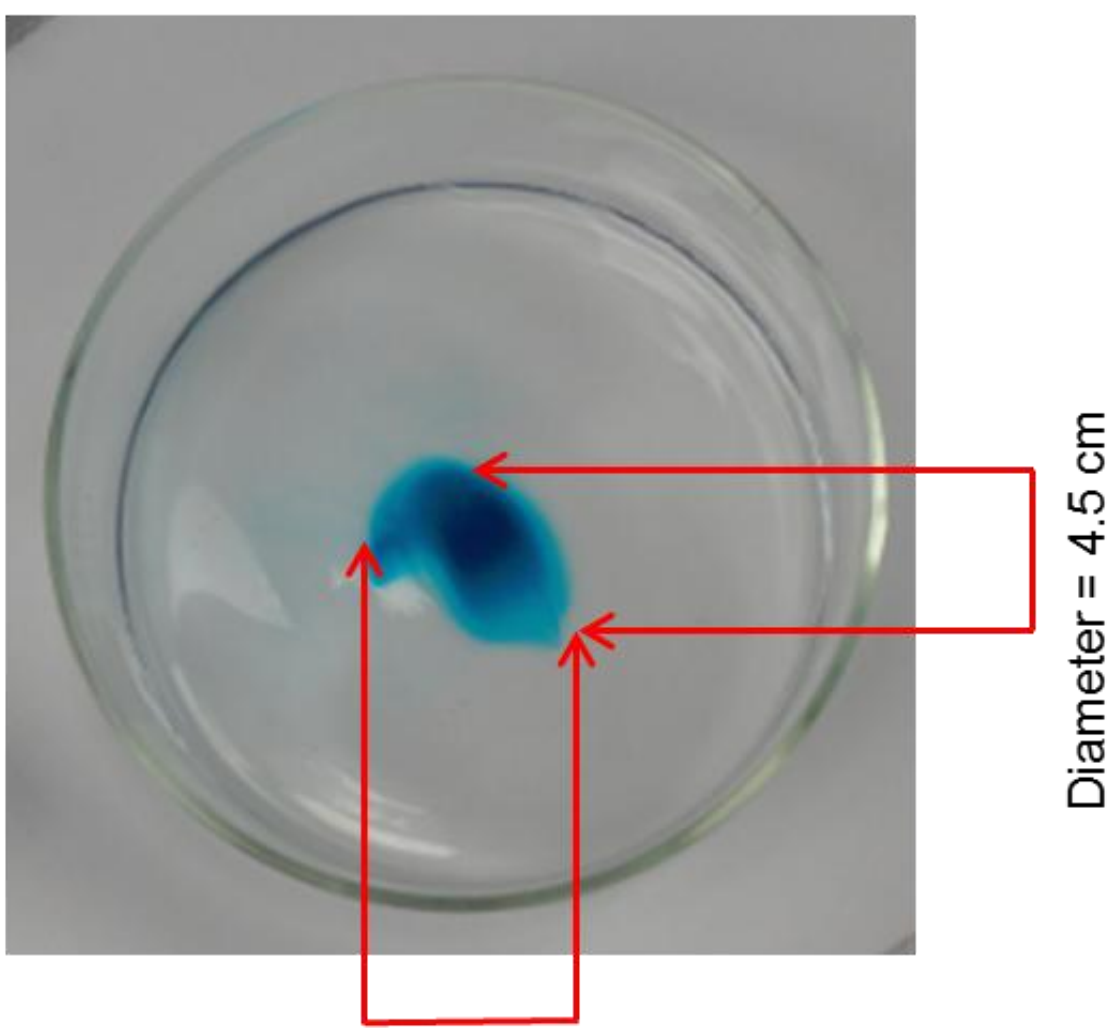

Diameter $=5 \mathrm{~cm}$

Spread of dye from center $=(5+4.5) / 4=2.4 \mathrm{~cm}$

Figure 5-2: Experimental Setup at time $=2 \mathrm{~min}$ 
The results obtained from the experiment appeared to be reasonable - observe the parabolic profile. The diffusion rate seemed to be rapid at the beginning and progressively fell as the dye-particles approached the external boundary. This behavior was consistent to the results obtained from the numerical approach - see Figure 5-4, and 0.

The next step was to develop numerical codes to solve the 1D diffusion-equation in the radial direction. An in-depth analysis of this procedure is included in 0 . The idea was to plot distance vs. time curves for varying diffusivity values and determine the curve that closely matched the physical experiment. Five different diffusivity values were utilized to generate five curves. The diffusivity values used were 1e-7, 3.25e-7, $5.5 \mathrm{e}-7,7.7 \mathrm{e}-7$, and $1 \mathrm{e}-6 \mathrm{~m}^{2} / \mathrm{s}$; see Figure $5-4$. It was concluded that the physicalexperiment matched the curve generated using the diffusivity value $5.5 \mathrm{e}-7 \mathrm{~m}^{2} / \mathrm{s}$; see Figure 5-4.

While actively performing the experimental work to determine the dye diffusivity, more information regarding the dye became available. It was determined that the dye utilized in the original experiment ${ }^{[6]}$ belonged to the class of food-coloring-dyes known as Brilliant Blue. On searching for more information, it was reported in one source $^{[22]}$ that diffusivity values close to $1 \mathrm{e}-10 \mathrm{~m}^{2} / \mathrm{s}$ could be acceptable for dyes belonging to the Brilliant-Blue's family.

As a result of the additional information becoming available, both the results $(5.5 \mathrm{e}-7$ and $1 \mathrm{e}-10 \mathrm{~m}^{2} / \mathrm{s}$ ) were used in the next step of the procedure to generate mass-fraction values. 


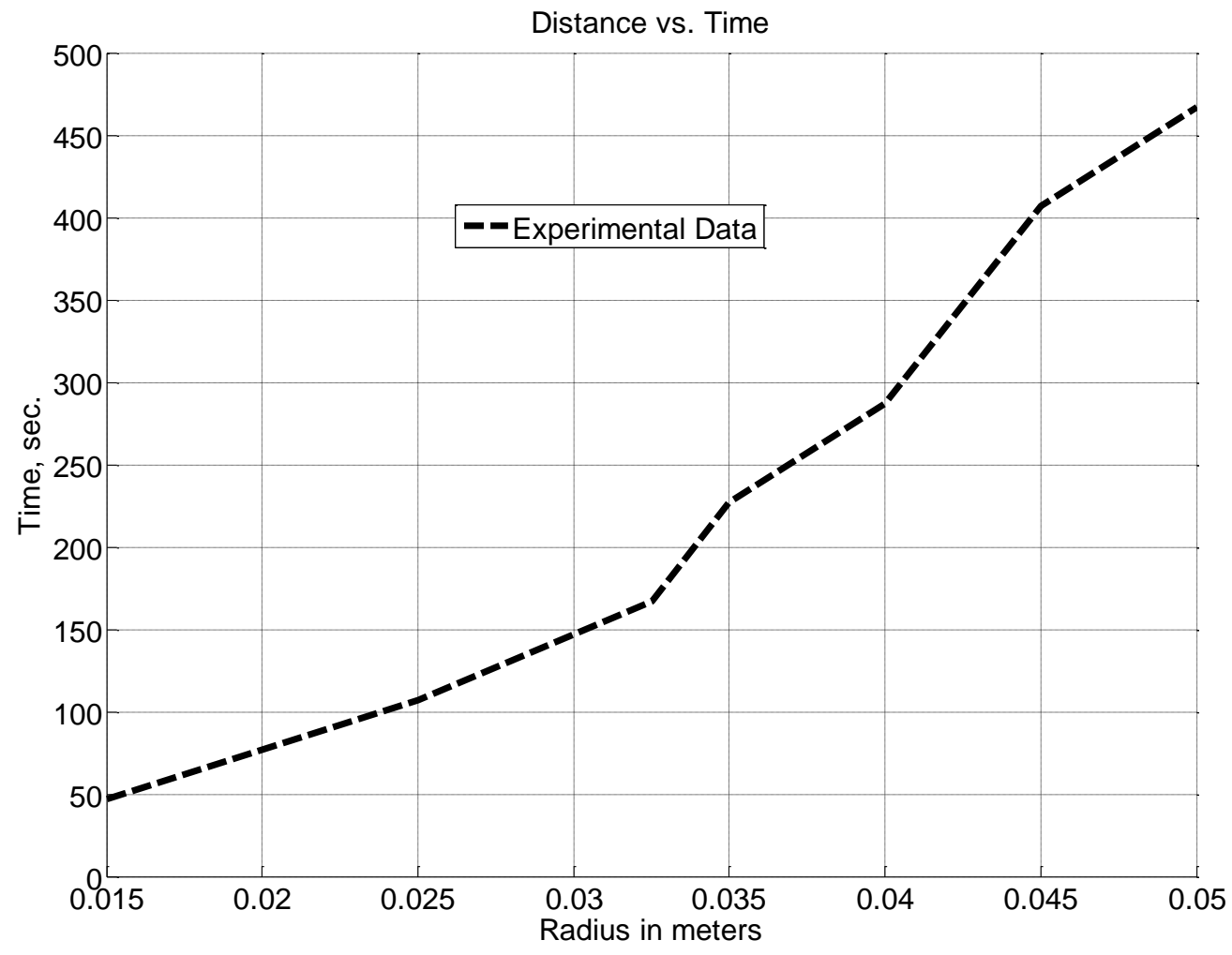

Figure 5-3: Experimental Results 


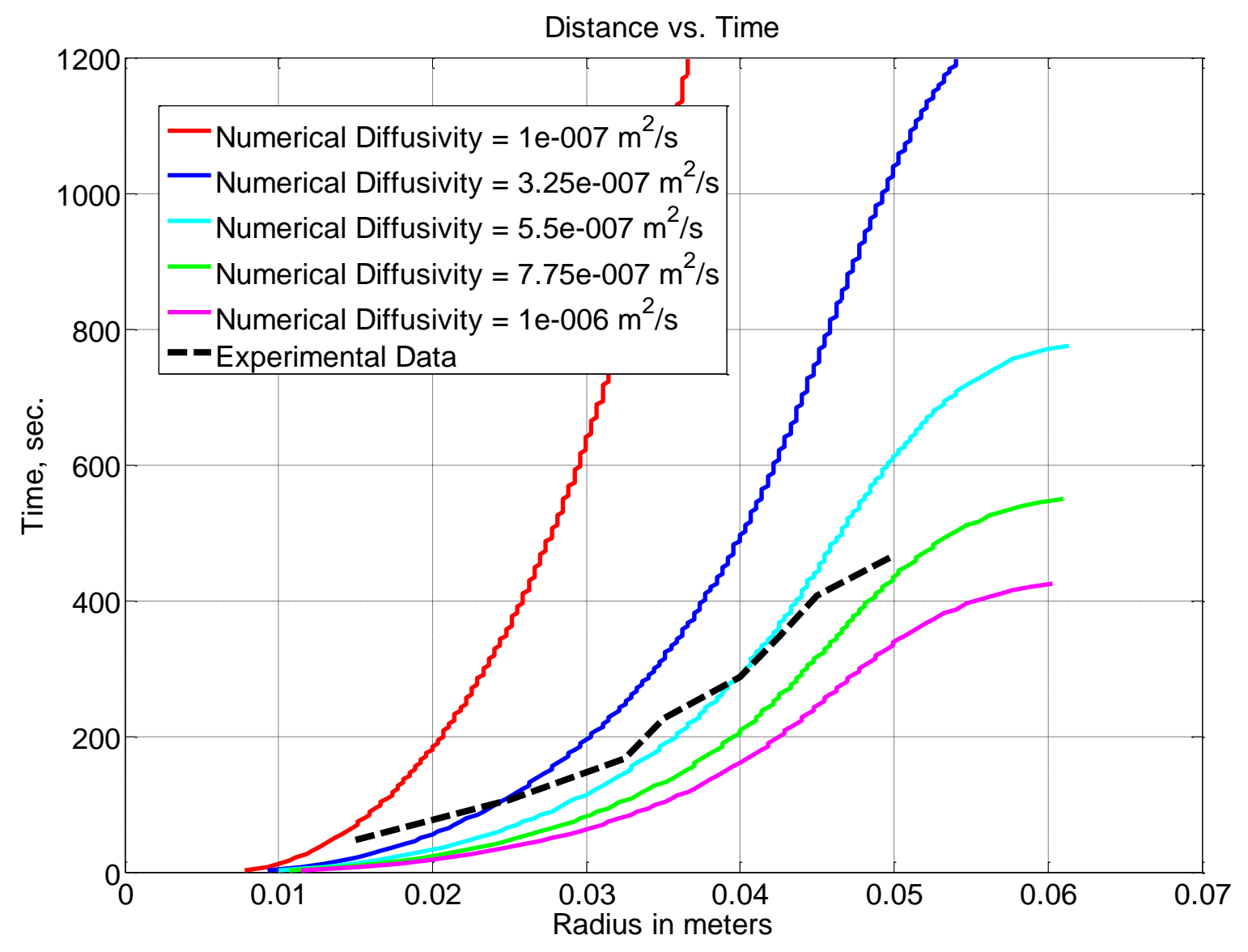

Figure 5-4: Experimental vs. Numerical results 


\subsection{Re-solving the scalar transport equation}

The next step involved solving the scalar transport equation (Eq. 1) utilizing the improved diffusivity vales - obtained from the experiment and literature. It was expected that the resulting mass-fraction values would indicate mixing.

Since the case files were readily available (in ANSYS Fluent) from the previous runs, setting the new diffusivity value and generating results were straightforward.

It may be noted here that the streamtrace results do not significantly change for varying diffusivity values - as diffusivity values only contribute to the scalar transport equation (Eq. 1) and not the Navier-Stoked equation (Eq. 3).

Figure 5-5 (i) represents the qualitative snapshot obtained from the original experiment ${ }^{[6]}$. Figure 5-5 (ii) indicates the contour generated using diffusivity $=1 \mathrm{e}$ $15 \mathrm{~m}^{2} / \mathrm{s}$. The result shown here was presented earlier (Figure 4-5) and is only repeated here for the reader's convenience. Figure 5-6 (i) represents the contour generated using diffusivity $=5.5 \mathrm{e}-7 \mathrm{~m}^{2} / \mathrm{s}-$ mixing appears to have been accomplished. Figure 5-6 (ii) shows the contour generated using diffusivity $=1 \mathrm{e}-10 \mathrm{~m}^{2} / \mathrm{s}$. The unmixed fluids exiting the system can be seen from the varying colors at the outlet. As expected, the mass-fraction data obtained using diffusivity $=5.5 \mathrm{e}-7 \mathrm{~m}^{2} / \mathrm{s}$ indicates complete mixing at the outlet - see Figure 5-6 (i) and Figure 5-8 (i). However, even though the qualitative-data ${ }^{[6]}$ - see Figure 5-5 (i) - indicated mixing at the outlet, there exists noticeable difference between the profiles. In the qualitative plot, mixing appears to happen progressively. This can be seen from the eventual blurring of the interface separating either fluid - notice the blue and white interface in Figure 5-5 (i). However, the contour generated with $\mathrm{D}=5.5 \mathrm{e}-7 \mathrm{~m}^{2} / \mathrm{s}$ indicates mixing to have been accomplished close to the inlet; see Figure 5-6 (i). On immediate consideration it might appear that the diffusivity value obtained from the experiment $(D=5.5 \mathrm{e}-7$ $\mathrm{m}^{2} / \mathrm{s}$ ) could possibly be erroneous. However, this is not the case. From previous intuition regarding the numerical errors accompanying the scalar-transport equation, 
this discrepancy can be addressed. The numerical-error in system could be dominant and have contributed to premature mixing.

Another interesting development was the relative similarity between the results obtained from utilizing diffusivity values $1 \mathrm{e}-10 \mathrm{~m}^{2} / \mathrm{s}$ and $1 \mathrm{e}-15 \mathrm{~m}^{2} / \mathrm{s}-$ see Figure $5-5$ (ii) and Figure 5-6 (ii). The similarity was further confirmed by the profiles generated at the inlet and outlet; see Figure 5-7 (i) and Figure 5-8 (i).

Figure 5-7 (i) represents the inlet-profile of the mass-fraction values obtained from solving the scalar transport equation utilizing varying diffusivity coefficients $-1 \mathrm{e}-10$, $5.5 \mathrm{e}-7,1 \mathrm{e}-15 \mathrm{~m}^{2} / \mathrm{s}$. Figure $5-7$ (ii) shows a magnified plot of the region enclosed within the circle in (i). Figure 5-7 (iii) indicates the location at which the profile-data were extracted. Description: Figure 5-8 (i) represents the outlet-profile of the massfraction values obtained from solving the scalar transport equation utilizing varying diffusivity coefficients $-1 \mathrm{e}-10,5.5 \mathrm{e}-7,1 \mathrm{e}-15 \mathrm{~m}^{2} / \mathrm{s}$. Figure $5-8$ (ii) shows a magnified plot of the region enclosed within the circle in (i). Figure 5-8 (iii) indicates the location at which the profile-data were extracted. The most likely explanation for this behavior could be the unresponsiveness of the equation to diffusivity values lower than $1 \mathrm{e}-10 \mathrm{~m}^{2} / \mathrm{s}$. This was reasonable considering how minuscule $1 \mathrm{e}-15 \mathrm{~m}^{2} / \mathrm{s}$ appears to be in the context of the experiment. However, in order not to negate other existing possibilities that might contribute to this observation, the reader is advised to consider this conclusion preliminary and within the scope of the current experiment. 
(i)

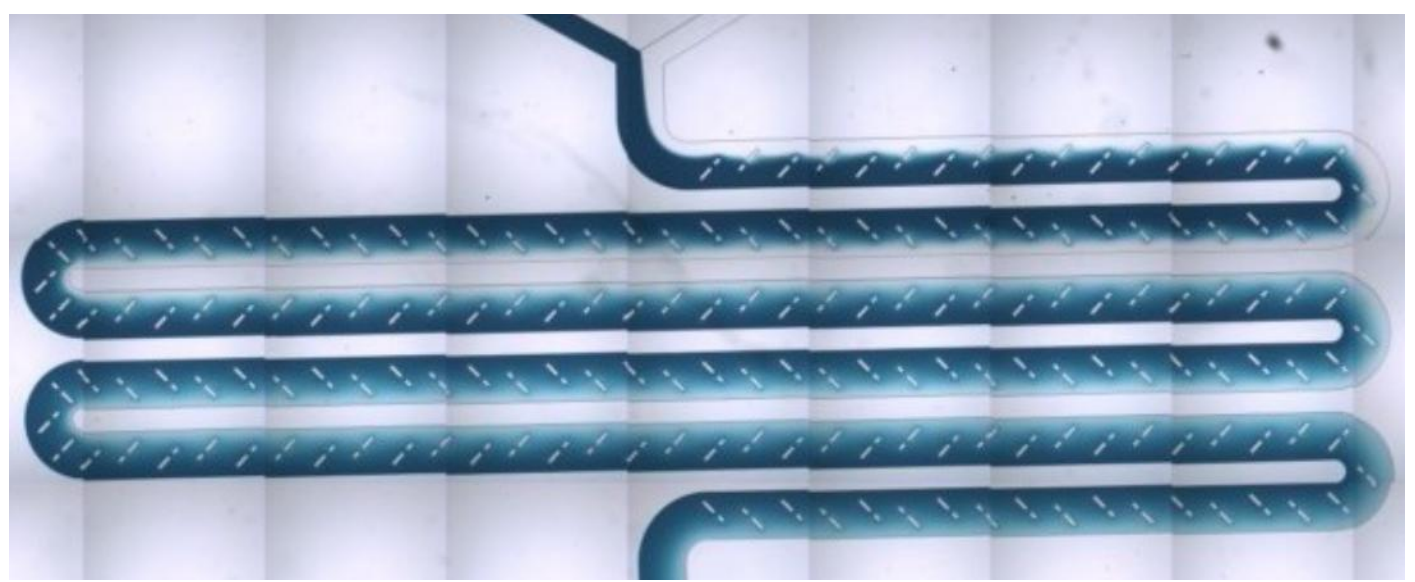

(ii)

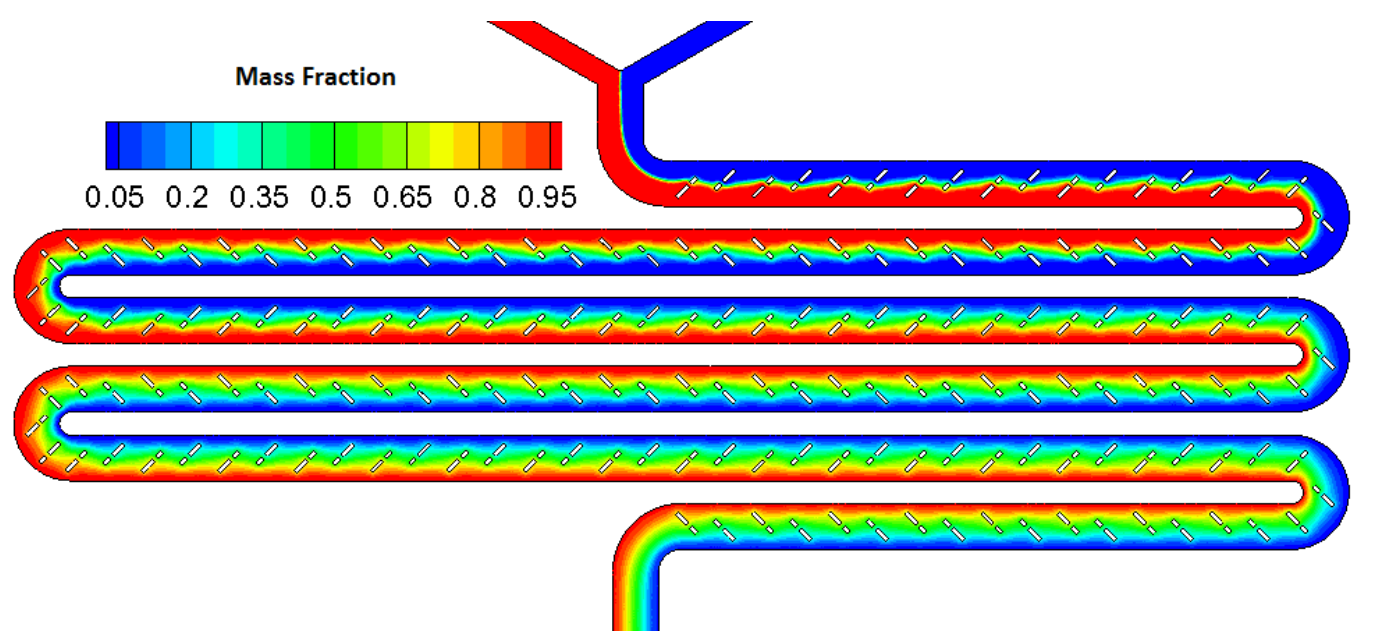

Figure 5-5 - Qualitative vs. Mass-Fraction for Multiple-bend-geometry 
(i)

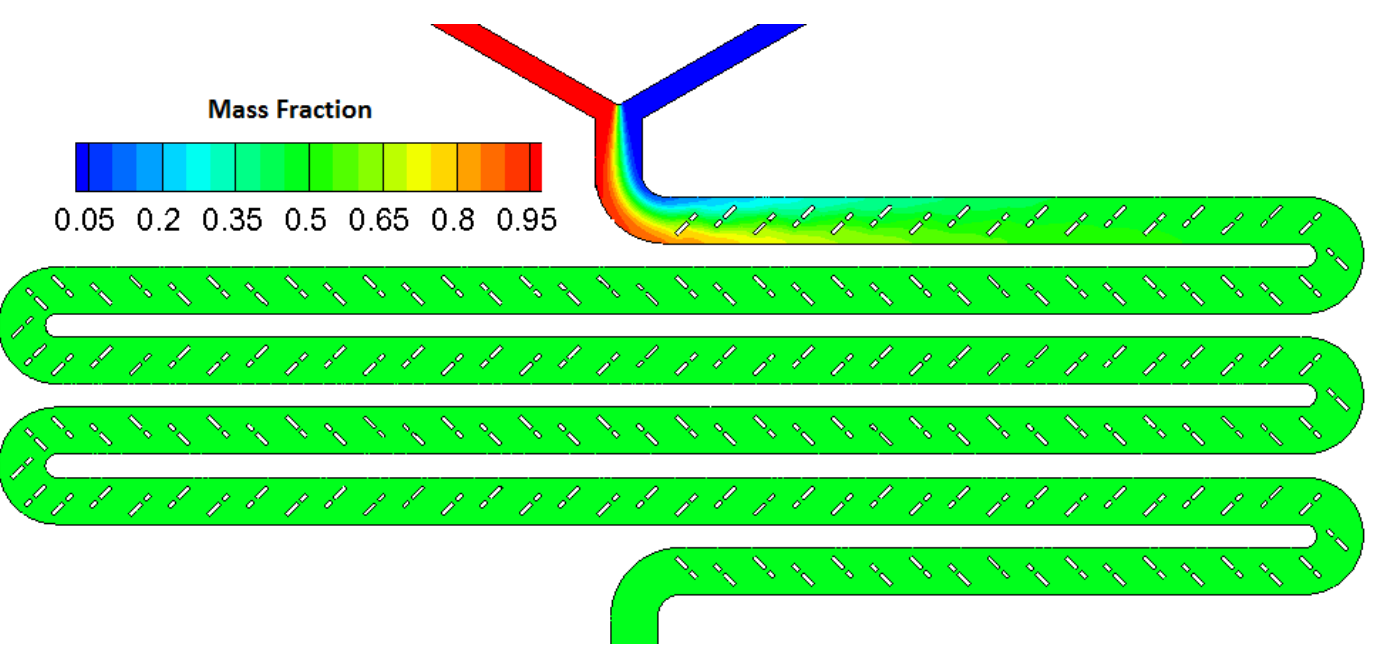

(ii)

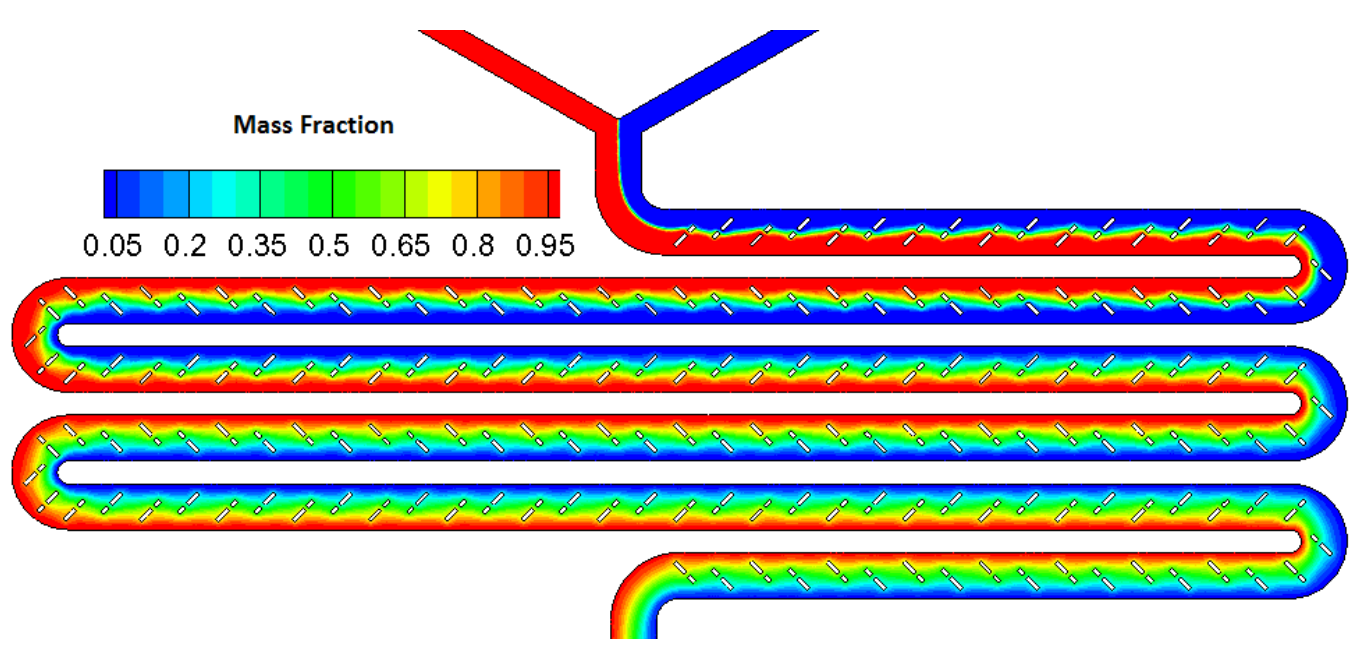

Figure 5-6 - Mass-Fraction data for varying diffusivities 
(i)

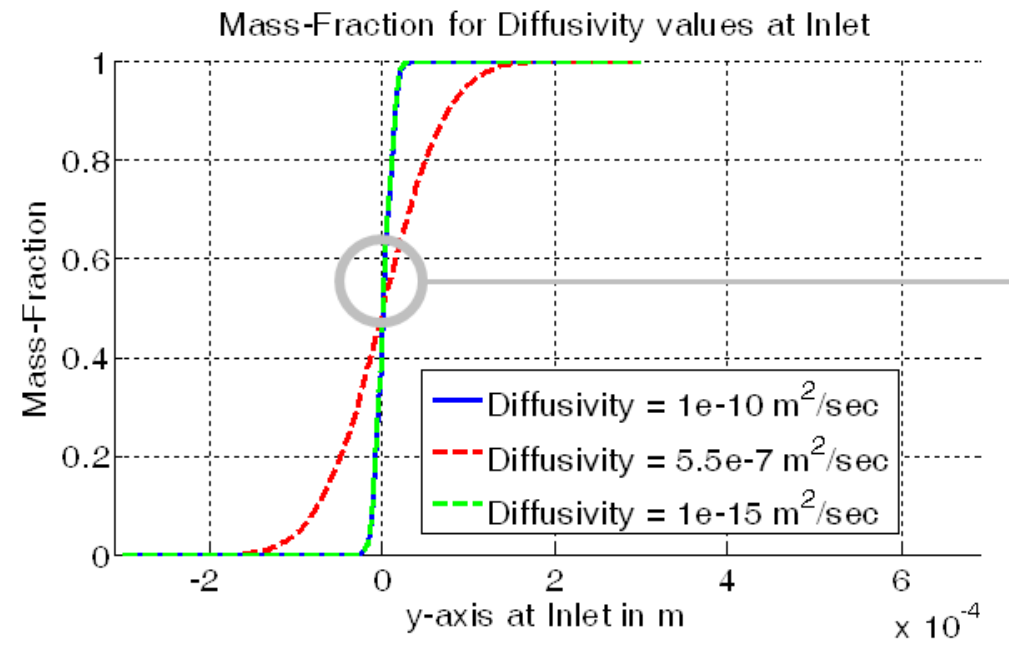

(ii)

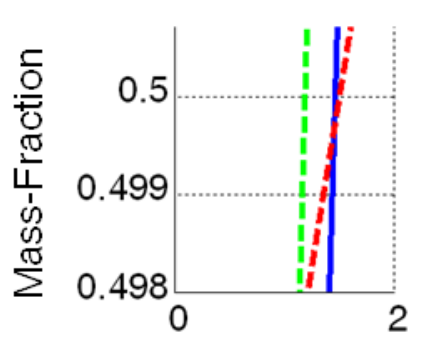

$y$-axis at Inlet in $\mathrm{m} \times 10^{-4}$

(iii)

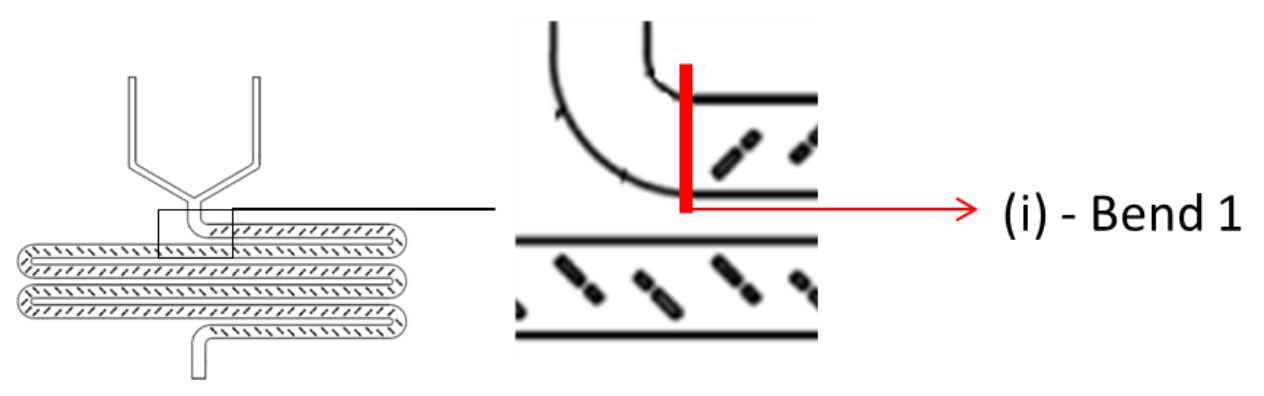

Figure 5-7 - Inlet profile for different diffusivities 
(i)

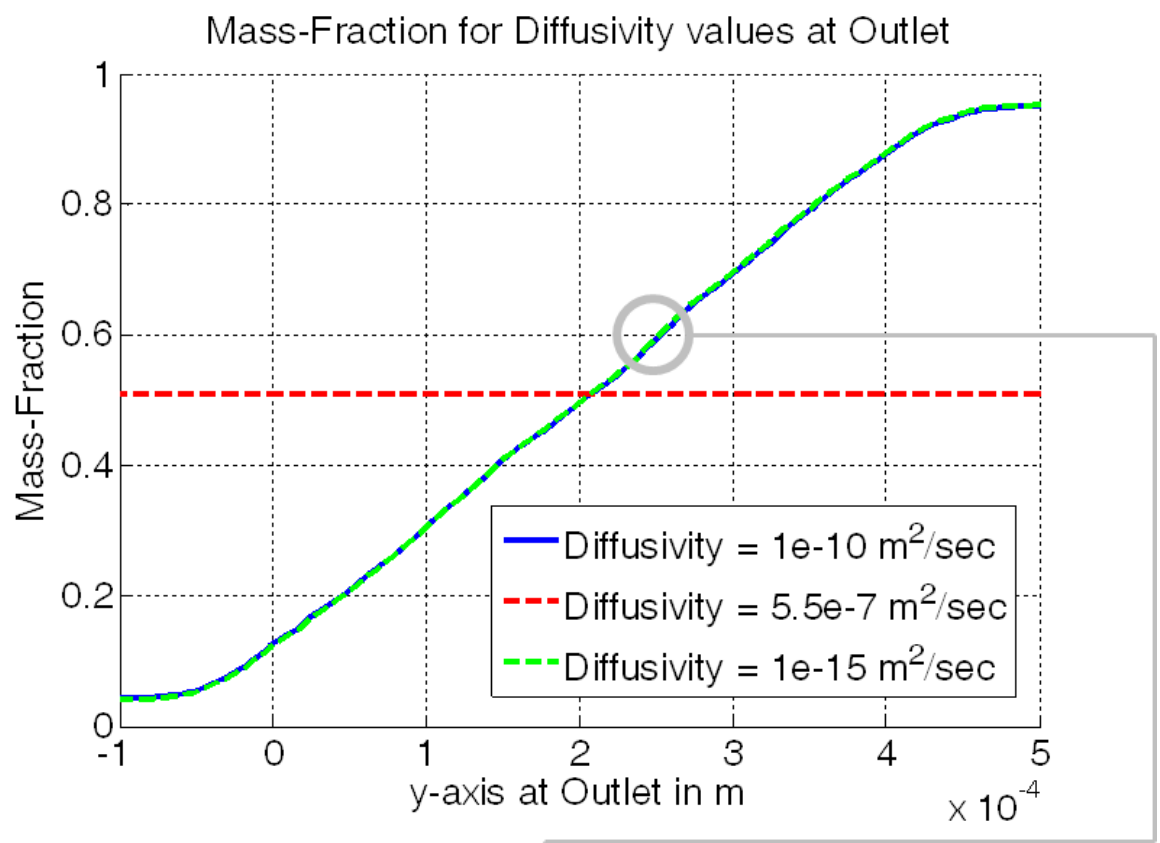

(ii)

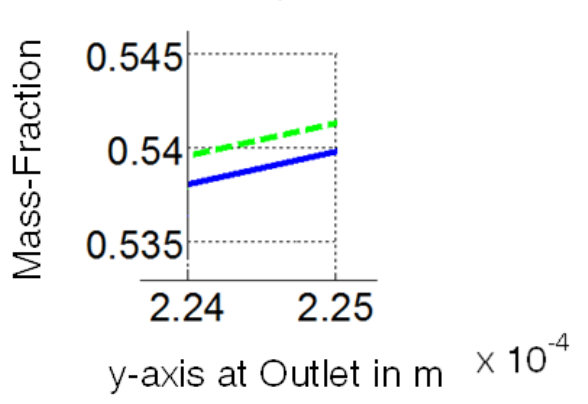

(iii)
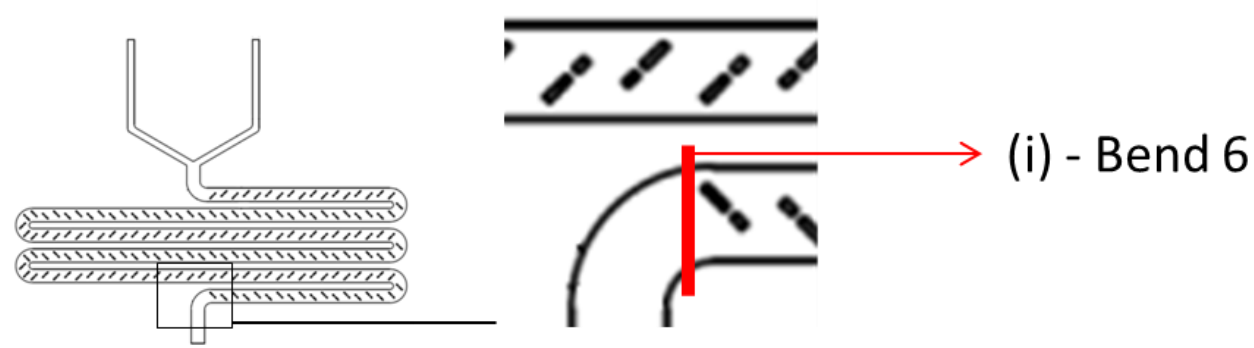

Figure 5-8 - Outlet profile for different diffusivities 
The experiments and simulations were conducted assuming Reynolds numbers lower than 50 - for microchannels ${ }^{[15]}$ - would result in similar data-outputs. The results for the HGB and multiple-bend geometry both were generated with $\mathrm{Re}=1$. However, the actual multiple-bend geometry configuration was solved with $\operatorname{Re}=0.1-$ corresponding to a flow rate $=1 \mu$. As a result, it was required to match the Reynolds numbers and run the simulations to check and confirm ideal behavior.

Figure 5-9 (i) presents mass-fraction contours obtained with diffusivity very close to zero $-1 \mathrm{e}-15 \mathrm{~m}^{2} / \mathrm{s}$. And, Figure 5-9 (ii) presents the mass-fraction contours obtained with diffusivity equal to $5.5 \mathrm{e}-7 \mathrm{~m}^{2} / \mathrm{s}$. It is clearly apparent that at zero diffusivity the fluids remain unmixed as they exit the system. However, when the diffusivity increases, mixing is very intense to the point of mass-fraction values close to 0.5 even apparent close to the inlet. This observation - at least based on the simulation indicates mixing in the multiple-bend geometry to be occurring as a result of the dyediffusivity and not as a result of the geometric-constraints. For the readers reference, a picture from the experiment is shown in Figure 5-9 (iii).

Finally, the changes apparent in the simulations that might be dependent on the Reynolds numbers are discussed. The simulations were carried out with dyediffusivity equal to $5.5 \mathrm{e}-7 \mathrm{~m}^{2} / \mathrm{s}$. The results are fairly similar that were expected - see Figure 5-10 (i), (ii), and (iii). However, one minor difference that stands out is the extent of the unmixed fluids occupying the geometry until mixing begins to appear. For the result with $\mathrm{Re}=50$, the convective effects are more dominant compared to diffusive effects and the unmixed-fluid extend out the farthest - Figure 5-10 (i). In contrast for the results with $\mathrm{Re}=1$, the convective effects are less dominant and the unmixed fluids barely extend out beyond the inlets. Despite the differences, it may be noted that the region where differences are dominant is within a half-length distance occupied by the first bend. The entire geometry consists of about 6 bends. Thus, a difference at just half-a-bend distances is permissible within the current-context of the study. 
(i)

Mass-Fraction

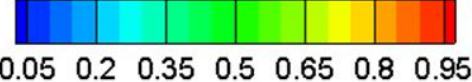

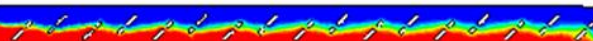

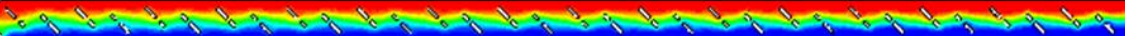

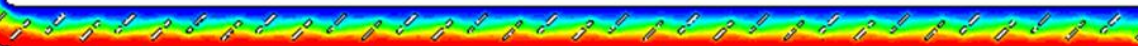

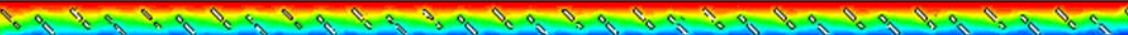

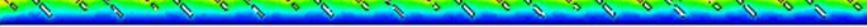

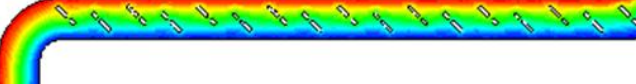

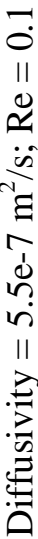

(ii)

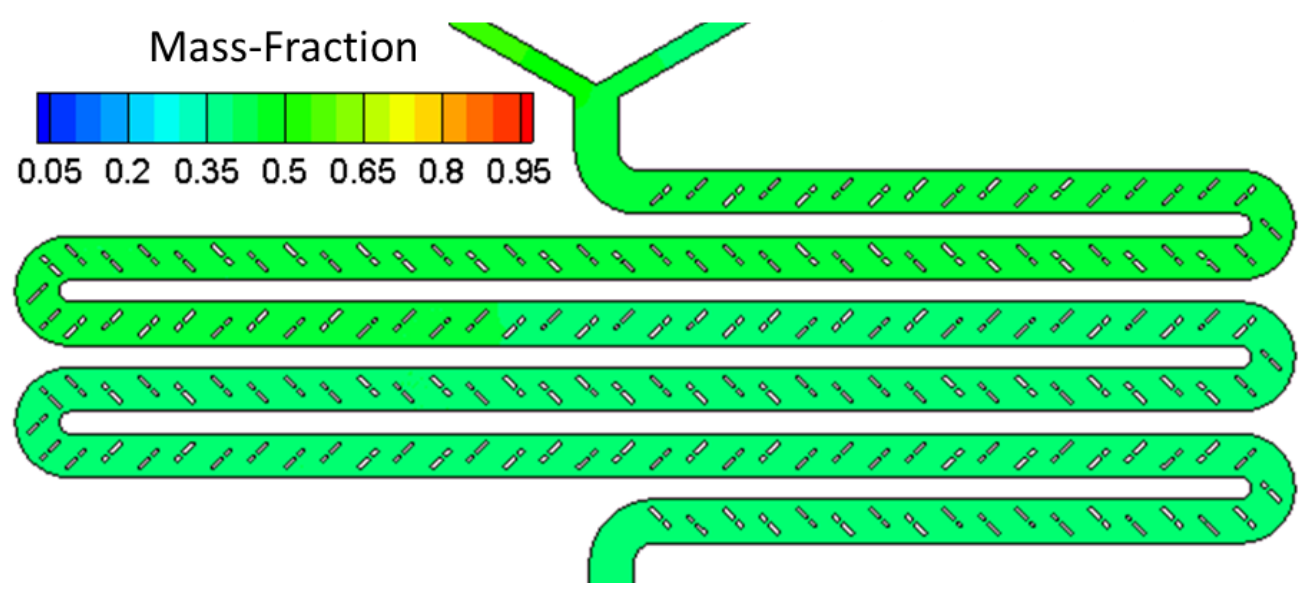

(iii)

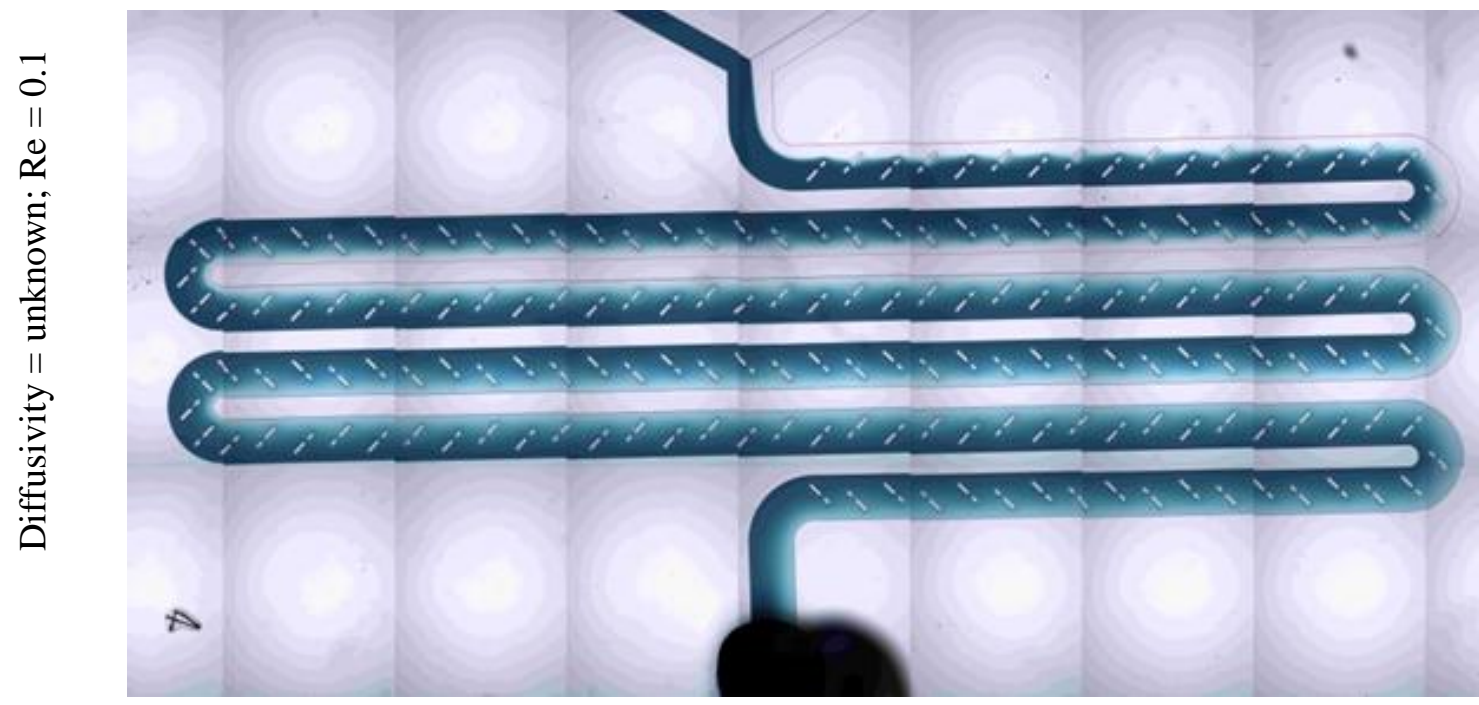

Figure 5-9: Matching Reynolds number with Experiments and Simulations 
(i)

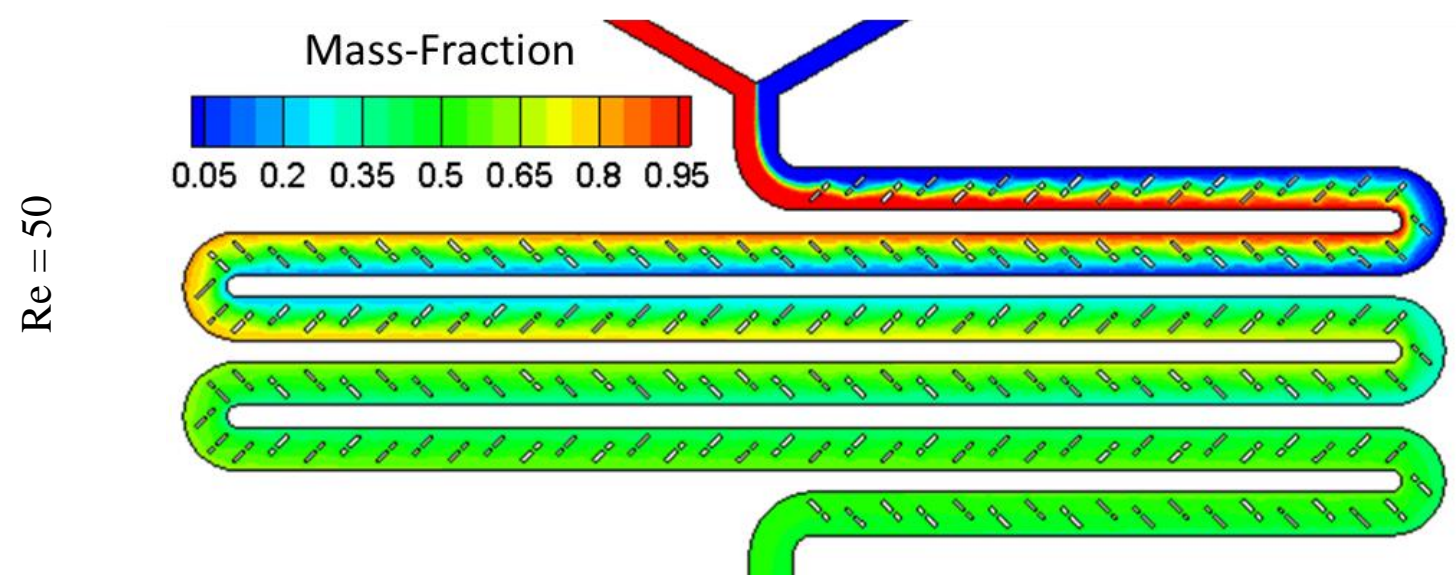

(ii)

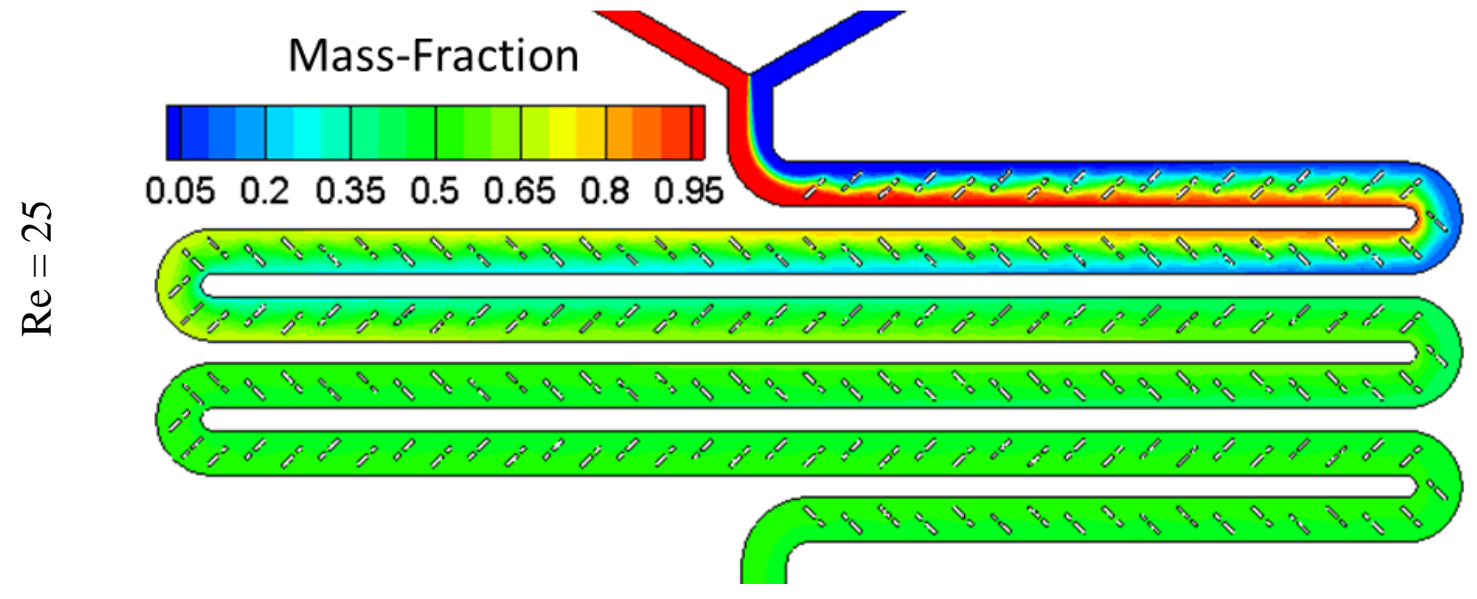

(iii)

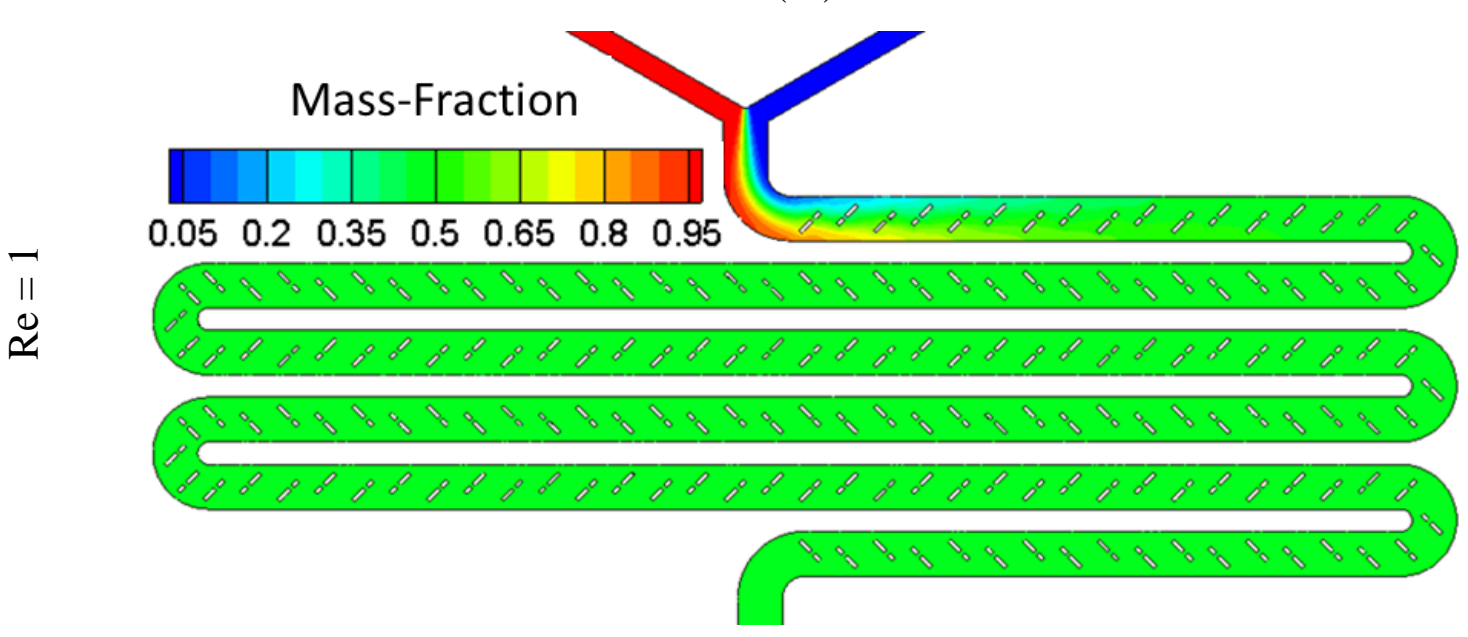

Figure 5-10: Flow with $\operatorname{Re}=1,25$, and 50; with diffusivity $=5.5 \mathrm{e}-7 \mathrm{~m}^{2} / \mathrm{s}$ 


\section{Chapter 6: CONCLUSIONS}

The objective of this thesis was to study a newly proposed method to quantify mixing in microchannles. The method utilized the streamtrace idea that has been used in the past $^{[7]}$ to analyze mixing in microchannles. The streamtrace method utilizes the velocity field data that was obtained by solving the Navier-stokes equation. The massfraction values were also calculated in order to compare results obtained from the streamtrace method. The mass-fraction data were obtained by solving the scalartransport equation.

Two geometries were considered and analyzed using the streamtrace method. The first geometry had been extensively studied in the past and was obtained from literature ${ }^{[15]}$. On thoroughly performing detailed analysis, it was apparent that the streamtrace method generated results far more superior to the mass-fraction results. This was expected as the scalar-transport equation suffered from numerical errors arising within itself in addition to those present from solving the Navier-stokes equation - the velocity field. Also, the steamtrace method successfully translated qualitative data to quantitative data.

The second geometry considered was developed at WVU ${ }^{[6]}$. The streamtrace method was not very efficient in producing good results. Also, the mass-fraction data obtained from solving the scalar-transport equation indicated unmixed fluids exiting the system. This was a discrepancy as the qualitative plots available from experiments ${ }^{[6]}$ indicated mixed fluids exiting the system. The unexpected development here was that mass-fraction values indicated unmixed fluids. It was expected that mass-fraction values would suffer from numerical errors to the extent of indicating mixed fluids exiting the system. Hence, further tests had to be performed to resolve the discrepancy.

Since the streamtrace and mass-fraction procedures remained similar for both the geometries, it was determined that the qualitative information from the experiment ${ }^{[6]}$ 
needed further investigation. On further analysis, it became apparent that the diffusivity value used in the numerical simulations was different from that which represented the dye in the experiment. In fact, the diffusivity of the dye in the experiment was unknown. Hence, it was determined to identify the diffusivity of the dye and subsequently recalculate the mass-fraction values.

Determining the diffusivity of the dye was a two-pronged process involving contributions from both physical-experiments and numerical-simulations. Subsequently, the exact diffusivity of the dye was determined to be $5.5 \mathrm{e}-7 \mathrm{~m} / \mathrm{s}$. This value was used in the scalar-transport equation to generate mass-fraction data. This final result indicated better agreement with the qualitative information from the experiment ${ }^{[6]}$. 


\section{References}

[1] M. A. Burns et al., "An Integrated Nanoliter DNA Analysis Device," Science, vol. 282 , no. 5388 , pp. 484-487, 1998.

[2] H. S. C. S. A. Chou and S. Quake, "Proceedings of the National Academy of Sciences of the United States of America," The National Academy of Sciences, vol. 96, no. 1, pp. 11-13, Jan. 1999.

[3] Losey M., W. Schmidt M., A.Jensen, "Microfabricated Multiphase Packed-Bed Reactors: Characterization of Mass Transfer and Reactions," Industrial \& Engineering Chemistry Research, vol. 40, no. 12, pp. 2555-2562, 2001.

[4] Sammarco, T., S., Burns M., A.,, "Thermocapillary pumping of discrete drops in microfabricated analysis devices," AIChE, vol. 45, no. 2, pp. 350-356, 1999.

[5] A. D. Strook, "Chaotic Mixer for Microchannels," Science, vol. 295, no. 647, 2002.

[6] Y. Liu, Experimental results were provided by Dr. Yuxin Liu research group, 2011.

[7] Aubin, J., Fletcher, D., Bertrand, J. and Xuereb, C. , "Characterization of the Mixing Quality in Micromixers," Chemical Engineering \& Technology, vol. 26, no. 12, pp. 1262-1270, December, 2003.

[8] Teiji TAKIGAWA, Naoto OHMURA, Kentaro YAGYUU and Kunio KATAOKA, "Information Complexity of Laminar Chaotic Mixing Field Produced," Forma, vol. 15, no. 3, pp. 273-280, May 2000.

[9] INOUE, Y. HIRATA, Y., " Numerical Analysis of Chaotic Mixing in Plane Cellular Flow II Mixedness and Final Mixing Pattern," KAGAKU KOGAKU 
RONBUNSHU, vol. 26, pp. 31-39, 2000.

[10] A. Cantu-Perez, "Residence time distributions in microchannels: Comparison between channels with herringbone structures and a rectangular channel," Chemical engineering journal, vol. 160, no. 3, p. 834, 2010.

[11] Peter G. M. Kruijt,Oleksiy S. Galaktionov, Patrick D. Anderson,Gerrit W. M. Peters, Han E. H. Meijer, "Analyzing mixing in periodic flows by distribution matrices: Mapping method," AIChE Journal, vol. 47, no. 5, pp. 1005-1015, April 2004.

[12] J. Rafael Pacheco, Kang Ping Chen, Mark A. Hayes, "Rapid and efficient mixing in a slip-driven three-dimensional flow in a rectangular channel," Fluid Dynamics Research, vol. 38, no. 8, pp. 503-521, August 2006.

[13] Hengzi Wang, Pio Iovenitti, Erol Harvey, Syed Masood, "Numerical investigation of mixing in microchannels with patterned grooves," Journal of Micromechanics and Microengineering, vol. 13, no. 6, November 2003.

[14] Zalc, J. M., Szalai, E. S., Muzzio, F. J. and Jaffer, S., "Characterization of flow and mixing in an SMX static mixer," AIChE Journal, vol. 48, no. 3, p. 427-436, March 2002.

[15] A. D. Stroock, S. K. W. Dertinger, A. Ajdari, I. Mezić, H. A. Stone and G. M. Whitesides, "Chaotic Mixer for Microchannels," Science, pp. 647-651, Jan. 25, 2002.

[16] Guan Heng Yeoh, Kwok Kit Yuen, Computational Fluid Dynamics in Fire Engineering: Theory, Modelling and Practice, Burlington, MA: ButterworthHeinemann, 2009.

[17] T. T, "Finite volume TVD scheme on unstructured grids for space MHD simulations and space weather," in Proceeding of simulation school at Nagoya, 
Nagoya, 2002.

[18] H K Versteeg, W Malalsekera, An Introduction to Computational Fluid Dynamics The Finite Volume Method, Gosport: Oearson, 2007.

[19] Kang, T., G., Kwon, T., H., "Colored particle tracking method for mixing analysis of chaotic micromixers," Journal of Micromechanics and Microengineering, vol. 14, no. 7, p. 891, 2004.

[20] W. -. $\quad$ F. $\quad$ Dye, July 11 2012. [Online]. Available: http://www.walmart.com/ip/Great-Value-Assorted-Food-Color-Egg-Dye-1oz/10315298.

[21] G.W. Wake, G.N. Ivey, J. Imberger, N.R. McDonald, "The temporal evolution of a geostrophic flow in a rotating stratified basin," Dynamics of Atmospheres and Oceans, vol. Volume 39, no. 3-4, p. 189-210, May 2005.

[22] H.-J. V. K. R. R. Kasteel, "Effect of non-linear adsorption on the transport behaviour of Brilliant Blue in a field soil," European Journal of Soil Science, vol. 53, no. 2, pp. 231-240, June 2002.

[23] A. E. B. Jr., "ANALYSIS OF A CURVED BUOYANT JET IN AN ENCLOSURE USING LES," Morgantown, 2003.

[24] G. Hu, "TOWARDS LARGE EDDY SIMULATION OF DISPERSED GASLIQUID TWO-PHASE TURBULENT FLOWS," Morgantown, 2005.

[25] E. KARAISMAIL, "TRANSPORT PROCESSES IN LOW TURBULENCE FLOWS ASSOCIATED WITH ENCLOSING HOODS," Morgantown, 2011.

[26] I. Celik, A. Badeau Jr., , "Verification and Validation of DREAM Code," Morgatnwon, 2003. 
[27] "Mixing in the process industries," Chemical Engineering, vol. 101, no. 1, p. 10, 1994.

[28] Jones, S., W., Young, W., R.,, "SHEAR DISPERSION AND ANOMALOUS DIFFUSION BY CHAOTIC ADVECTION," JOURNAL OF FLUID MECHANICS, vol. 280, pp. 149-172, DEC 101994. 


\section{APPENDIX A : Solving Diffusion Equation Numerically}

The objective in this section was to numerically solve the $1 \mathrm{D}$ diffusion-equation. The equation is susceptible to change in the radial direction alone. Cylindrical-coordinates were applied in order to match the physical experiment.

The $1 \mathrm{D}$ diffusion-equation in the radial direction is shown in Eq. 8. The variables in the equation are described in Table A-1.

$$
\frac{\partial \theta}{\partial t}=\frac{D}{r} \frac{\partial\left(r \frac{\partial \theta}{\partial r}\right)}{\partial r}
$$

\section{Table A-1: Diffusion equation variables}

\begin{tabular}{|l|}
\hline - Concentration in $\mathrm{kg} / \mathrm{m}^{3}$ \\
\hline $\mathrm{T}$ - time \\
\hline $\mathrm{r}$ - radial-direction variable \\
\hline $\mathrm{D}$ - Diffusivity in $\mathrm{m}^{2} / \mathrm{s}$ \\
\hline
\end{tabular}

Figure A-1 describes the geometry conditions at time, $t=0$ is shown in the figure. The variables $\mathrm{rIC}$ and $\mathrm{rBC}$ are measured at an arbitrary time assumed to be at 0 seconds. The initial/boundary conditions for the geometry are described in Eq. 9 and 10. From Figure A-1, it may be noted that variable $r_{I C}$ indicates the spread of the dye (with coccentration $=1220 \mathrm{~kg} / \mathrm{m}^{3}$ ) shortly after it is introduced into the system - for the purpose of the experiment, the time was set to zero ( $\mathrm{t}=0$ seconds) at that instant. Variable $r_{B C}$ remains constant at all times and indicates the boundary of the system. The region enclosed within $r_{I C}$ and $r_{B C}$ is occupied by water with $=1000 \mathrm{~kg} / \mathrm{m}^{3}$ at the same moment $(t=0)$. 


$$
\begin{aligned}
& \theta\left(t=0,0 \leq r \leq r_{I C}\right)=1220 \frac{\mathrm{kg}}{\mathrm{m}^{3}} \\
& \theta\left(t=0, r_{I C} \leq r \leq r_{B C}\right)=1000 \frac{\mathrm{kg}}{\mathrm{m}^{3}}
\end{aligned}
$$

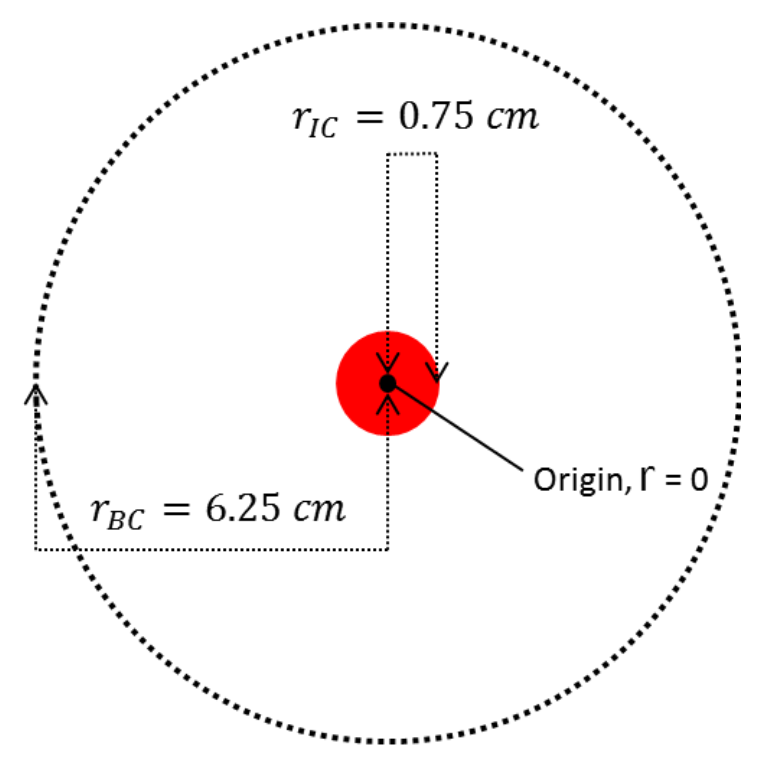

Figure A-1: Diffusivity Geometry and initial/boundary conditions

(i)

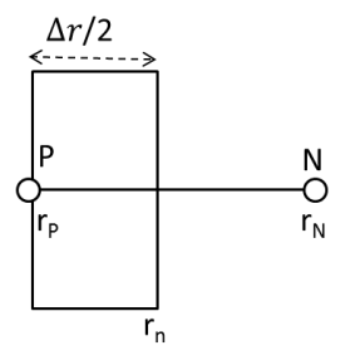

(ii)

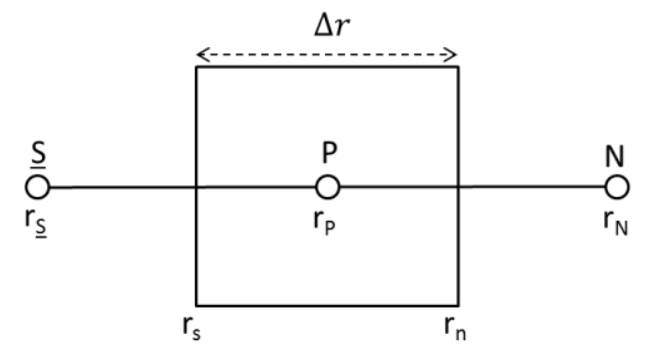

(iii)

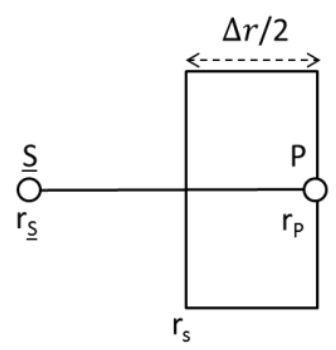

Figure A-2: Volume elements for discretization 
Figure A-2 describes the volume elements considered to discretize the diffusion equation. The south boundary (or the origin) uses the scheme in Figure A-2 (i). The north boundary (end) uses the scheme in Figure A-2 (iii). All other regions in the interior use the scheme in Figure A-2 (ii). It may be noted that the volume elements in reality are just area elements - as the spread of the dye is considered on a 2D plane with change occurring only in the radial direction.

The next step involved performing the numerical procedure to discretize the equation. The diffusion equation was integrated over a control volume and then over a suitable time interval. This step resulted in being able to predict the concentration at any location within the geometry at any given time. The equation describing the numerical process is described in detail; see Table A-2.

Following the calculations, the final equation critical to generating the relevant concentration values was obtained; see step 7 in Table A-2. This equation was obtained by employing a time-explicit integration. A time-explicitness condition utilizes the variable-values from the previous time-step to approximate the solution at the new-step. Implicit methods are typically preferred when available as the solution is more accurate and uses minimal processing-time. However, an explicit approximation is adequate for the current case as it did not involve convective components. In the absence of convective-components the difference between the discretization schemes is greatly reduced.

The process discussed thus far resulted in obtaining concentration values at the respective grid points for a given time. However, the final objective was to determine a radius - describing the spread of dye - at any given time. A simple approximation was used translate the concentration data into an equivalent radius data. An example concentration data is shown in Figure A-3. The concentration data from Figure A-3 is translated into a radius data as shown in Figure A-4. The Lambda curve approaches a minimum value at a given radius location - Figure A-4. This location was determined 
with a tolerance range of $2 \%$ and describes the spread of the dye at that instant - time $=720$ seconds in Figure A-4.

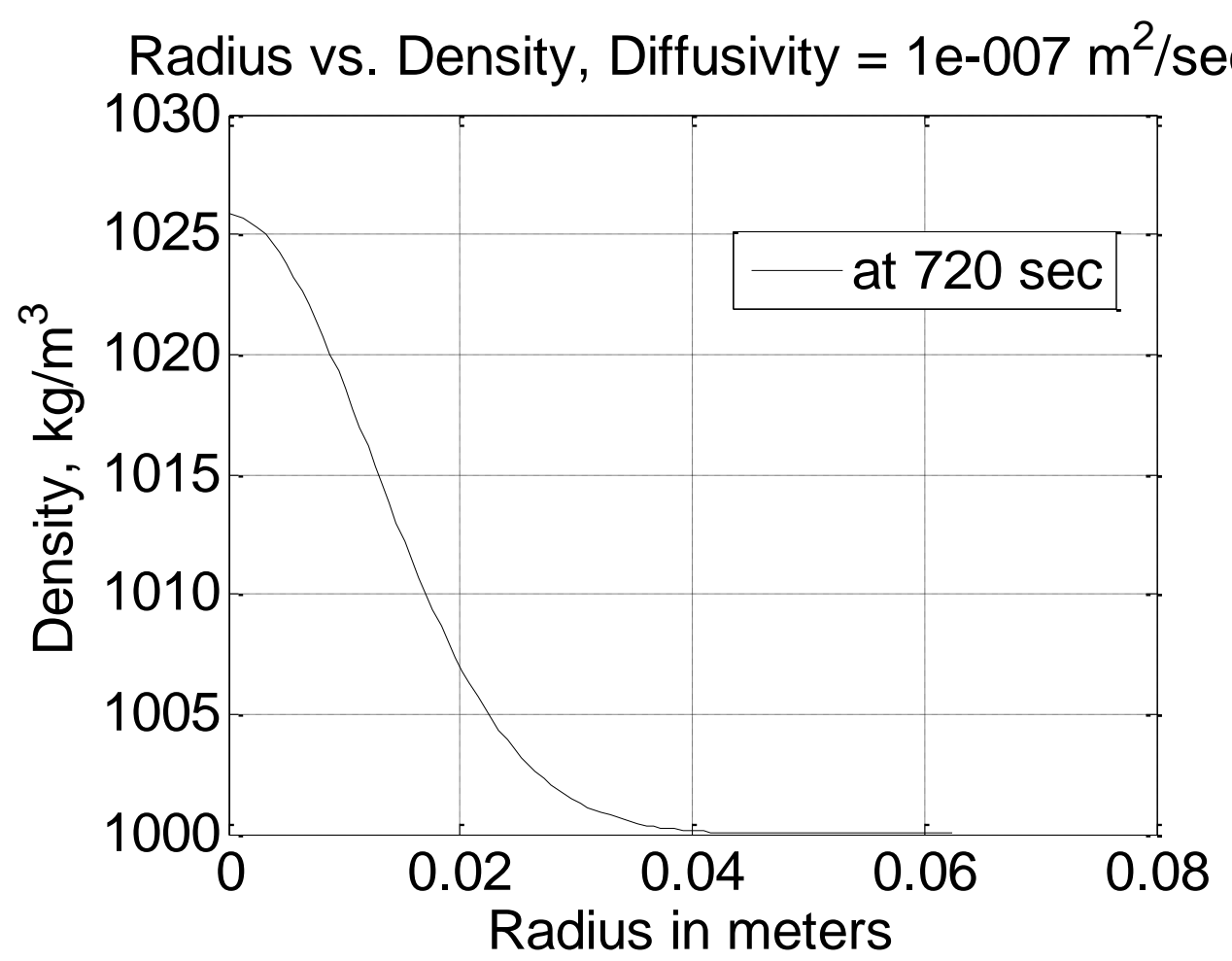

Figure A-3: Radius vs. Density at time 720 seconds for diffusivity $=1 \mathrm{e}-7 \mathrm{~m}^{2} / \mathrm{s}$ 


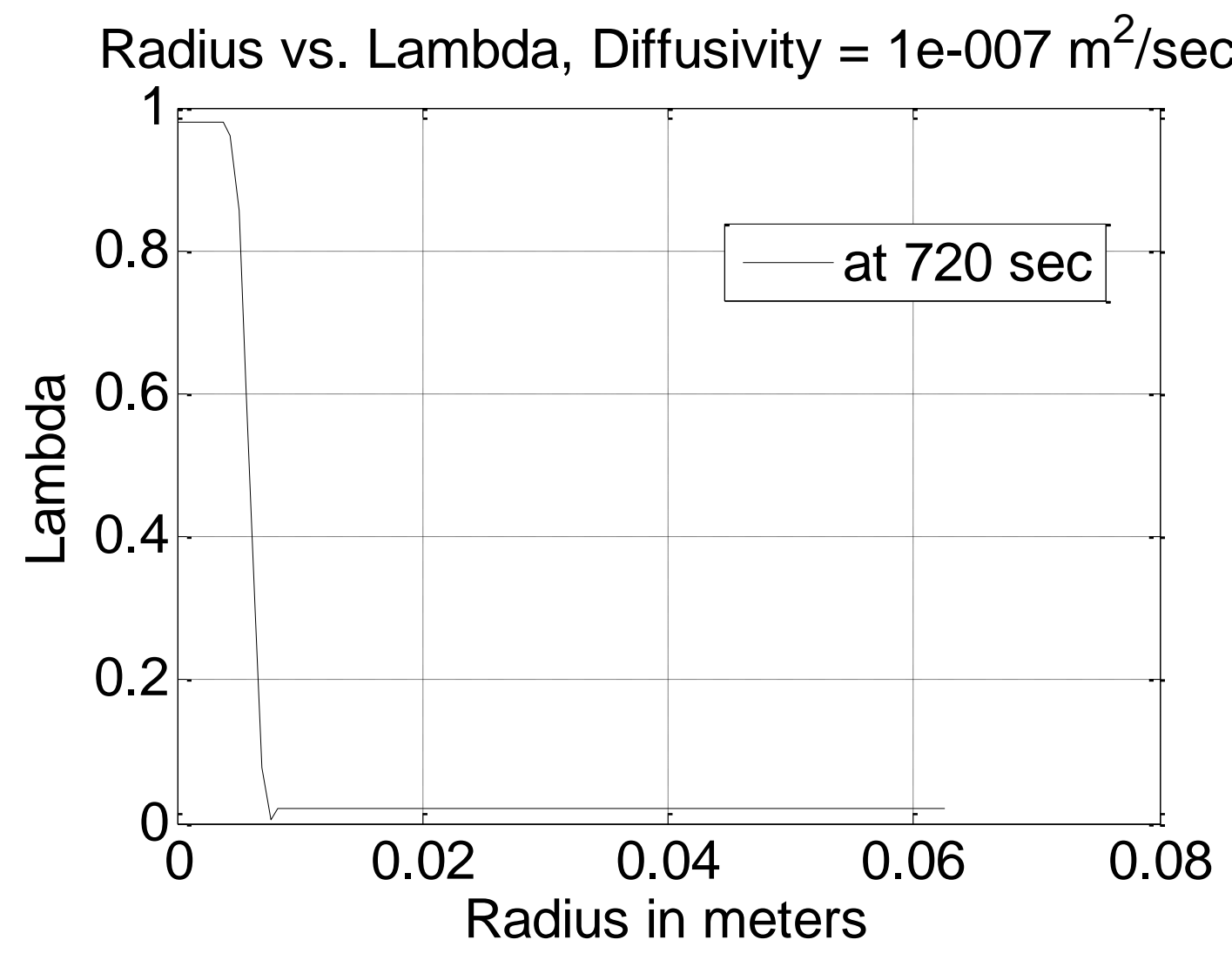

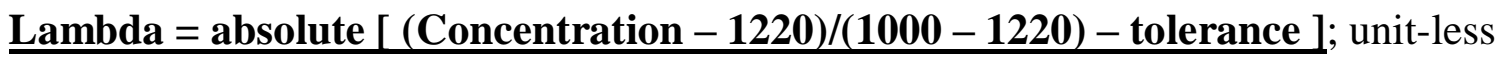
Where,

Tolerance $=0.02$, i.e. $2 \%$

$1220 \mathrm{~kg} / \mathrm{m}^{3}=$ original dye density $1000 \mathrm{~kg} / \mathrm{m}^{3}=$ clear water density

Concentration in $\mathrm{kg} / \mathrm{m}^{3}=$ a measure of concentration, varies with the radius

Figure A-4: Radius vs. Lambda at time 720 seconds for diffusivity $=1 \mathrm{e}-7 \mathrm{~m}^{2} / \mathrm{s}$ 
The final step was to code the numerical scheme in MATLAB and generate the necessary plots in order to compare results obtained from the physical experiment. The MATLAB code implemented for this purpose is shown in Table A-3.

The diffusion equation is relatively simple to solve numerically. Various instances of this exercise can be found in introductory CFD textbooks. The credibility of the data obtained from this procedure may be validated utilizing a simple rule of thumb. The 1D diffusion-equation is a simple PDE and hence its solution was expected to scale as a parabolic-profile. This simple check can be verified in Figure 5-4. In addition, the procedure's validity may further be accredited by the physical-experiment that was characterized by a parabolic-profile as well - see Figure 5-3. 
Table A-2: Detailed Numerical Method Procedure

Step 1: Integrating Diffusion equation over control-volume and time

$$
\int_{C V} \int_{t^{\circ}}^{t}\left[\frac{\partial \theta}{\partial t}=\frac{D}{r} \cdot \frac{\partial\left(r \frac{\partial \theta}{\partial r}\right)}{\partial r}\right] d V \cdot d t
$$

Step 2: Considering the LHS of the equation

$$
\int_{C V} \int_{t^{\circ}}^{t} \frac{\partial \theta}{\partial t}=\int_{r_{s}}^{r_{n}} \int_{t^{\circ}}^{t}\left(2 \pi r \cdot \frac{\partial \theta}{\partial t}\right) d r \cdot d t
$$

Step 3: Simplifying LHS of the equation

$\Rightarrow \int_{t^{\circ}}^{t} \pi\left(r_{n}^{2}-r_{s}^{2}\right) \cdot\left(\frac{\partial \theta}{\partial t}\right) d t=\pi\left(r_{n}^{2}-r_{s}^{2}\right) \cdot\left[\theta_{P}-\theta_{P}^{o}\right]$

Step 4: Considering the RHS of the equation

$$
\int_{C V} \int_{t^{\circ}}^{t}\left[\frac{D}{r} \cdot \frac{\partial\left(r \frac{\partial \theta}{\partial r}\right)}{\partial r}\right] d V \cdot d t=\int_{r_{s}}^{r_{n}} \int_{t^{\circ}}^{t}\left[\frac{D}{r} \cdot \frac{\partial\left(r \frac{\partial \theta}{\partial r}\right)}{\partial r}\right] \cdot 2 \pi r . d r . d t
$$

Step 5: Simplifying RHS of the equation

$$
\begin{aligned}
\Rightarrow 2 \pi D \int_{t^{\circ}}^{t}\left[r_{n} \cdot \frac{\left(\theta_{N}-\theta_{P}\right)}{\left(r_{N}-r_{P}\right)}-r_{s} \cdot \frac{\left(\theta_{P}-\theta_{S}\right)}{\left(r_{P}-r_{S}\right)}\right] d t \\
\quad=2 \pi D \cdot \Delta t\left[r_{n} \cdot \frac{\left(\theta_{N}-\theta_{P}\right)}{\left(r_{N}-r_{P}\right)}-r_{s} \cdot \frac{\left(\theta_{P}-\theta_{S}\right)}{\left(r_{P}-r_{S}\right)}\right]^{o} \quad \ldots \text { explicit }
\end{aligned}
$$

Step 6: Setting LHS = RHS

$\left(r_{n}^{2}-r_{s}^{2}\right) \cdot\left[\theta_{P}-\theta_{P}^{o}\right]=2 D \cdot \Delta t\left[r_{n} \cdot \frac{\left(\theta_{N}^{o}-\theta_{P}^{o}\right)}{\left(r_{N}-r_{P}\right)}-r_{s} \cdot \frac{\left(\theta_{P}^{o}-\theta_{S}^{o}\right)}{\left(r_{P}-r_{S}\right)}\right]$

Step 7: Rearranging the equation to solve for $\theta_{P}$, and grouping variables

$\theta_{P}=\frac{\left[a_{N} \theta_{N}^{o}+a_{S} \theta_{S}^{o}+\left(a_{P}-a_{N}-a_{S}\right) \cdot \theta_{P}^{o}\right]}{a_{P}}$

Step 8: Descriptions of the variables

where $a_{N}=\frac{r_{n}}{\left(r_{N}-r_{P}\right)}, a_{S}=\frac{r_{s}}{\left(r_{P}-r_{S}\right)}$ and, $a_{P}=\frac{\left(r_{n}^{2}-r_{s}^{2}\right)}{2 D . \Delta t}$ 
function [time_data] $=$ Diff_plot(D)

clear all;

close all;

clc;

$\mathrm{n}=100 ; \%$ the number of nodes $\ldots$

$\mathrm{Lf}=0.125 / 2 ; \%$ radius of domain in meters $\ldots$

$\mathrm{Lo}=0.0000001$

$\mathrm{dt}=1 ; \% \mathrm{dt}=60 \mathrm{sec}$

$\% \mathrm{D}=1 \mathrm{e}-8 ; \%$ Diffusivity

time_elapsed $=60 * 20 ; \% \mathrm{sec}$

$\mathrm{A}=2 * \mathrm{D} * \mathrm{dt} ; \%$

$\mathrm{nFIG}=5$;

$\mathrm{nDAT}=1000 ; \%$ no of data points to generate the final position vs time plot;

tol $=0.02 ; \% 2$ percent tolerance value to generate plot

N_boundary $=1000 ; \%$ densuty $\mathrm{kg} / \mathrm{m}^{\wedge} 3$, which is a North Boundary

initial_condition $=1220 ; \%$ desnity at node 1 is originally $1220 \mathrm{~kg} / \mathrm{m}^{\wedge} 3$

r_P $=[$ Lo :(Lf-Lo $) /(\mathrm{n}-1): \mathrm{Lf}]$;

r_N $=$ r_P $+($ Lf $-L o) /(n-1)$;

r_N(n) $=1 \mathrm{e}-8 ; \%$ North boundary does not exist...

r_S = r_P - (Lf-Lo)/(n-1);

r_S $(1)=0.98 \mathrm{e}-8 ; \%$ South boundary does not exist for node 1

$\mathrm{r} \_\mathrm{n}=\mathrm{r} \_\mathrm{P}+(\mathrm{Lf}-\mathrm{Lo}) /(\mathrm{n}-1) * 0.5$;

r_n $(n)=0.995 \mathrm{e}-8 ; \%$ north boundary does not exist... 


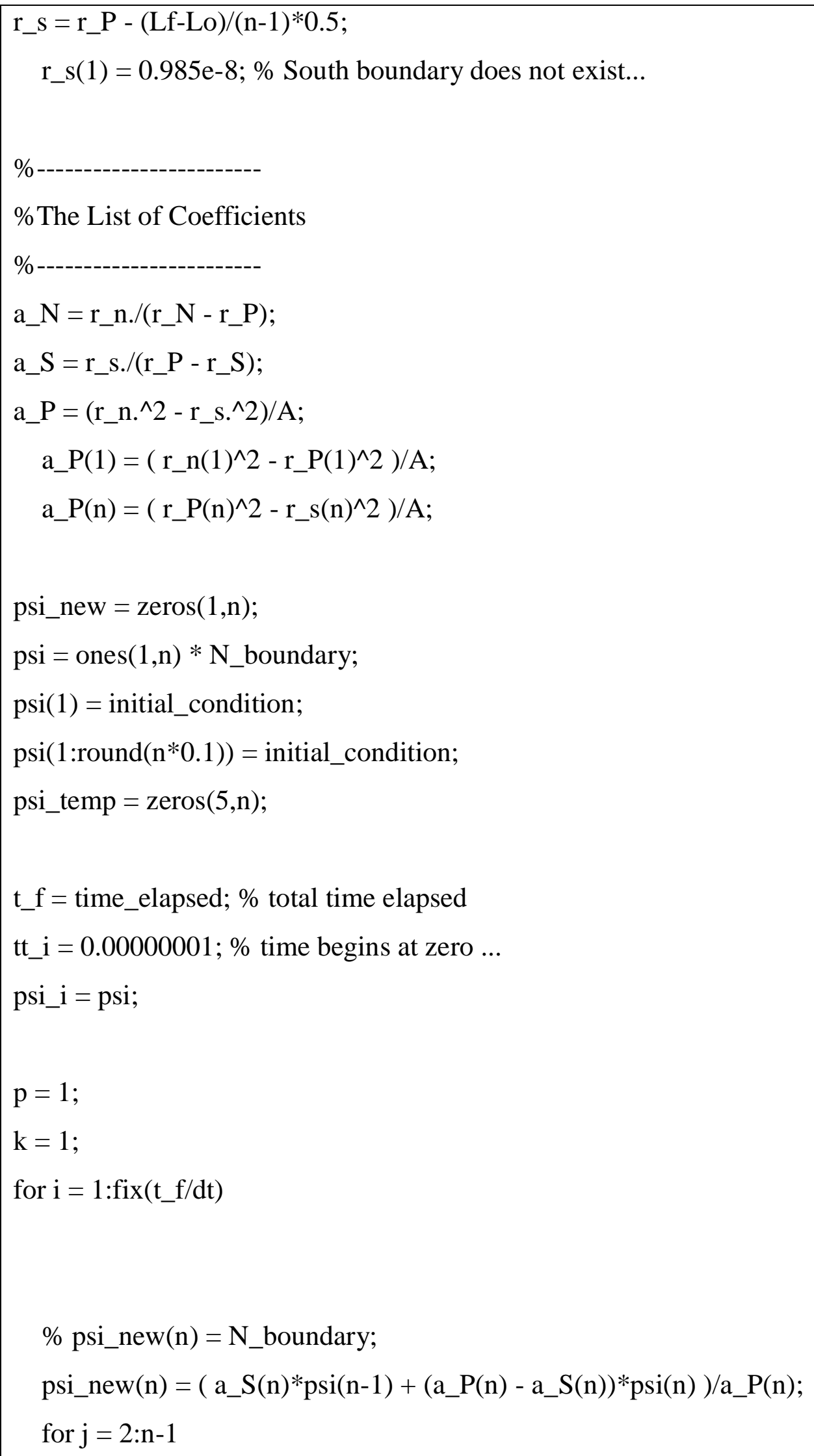




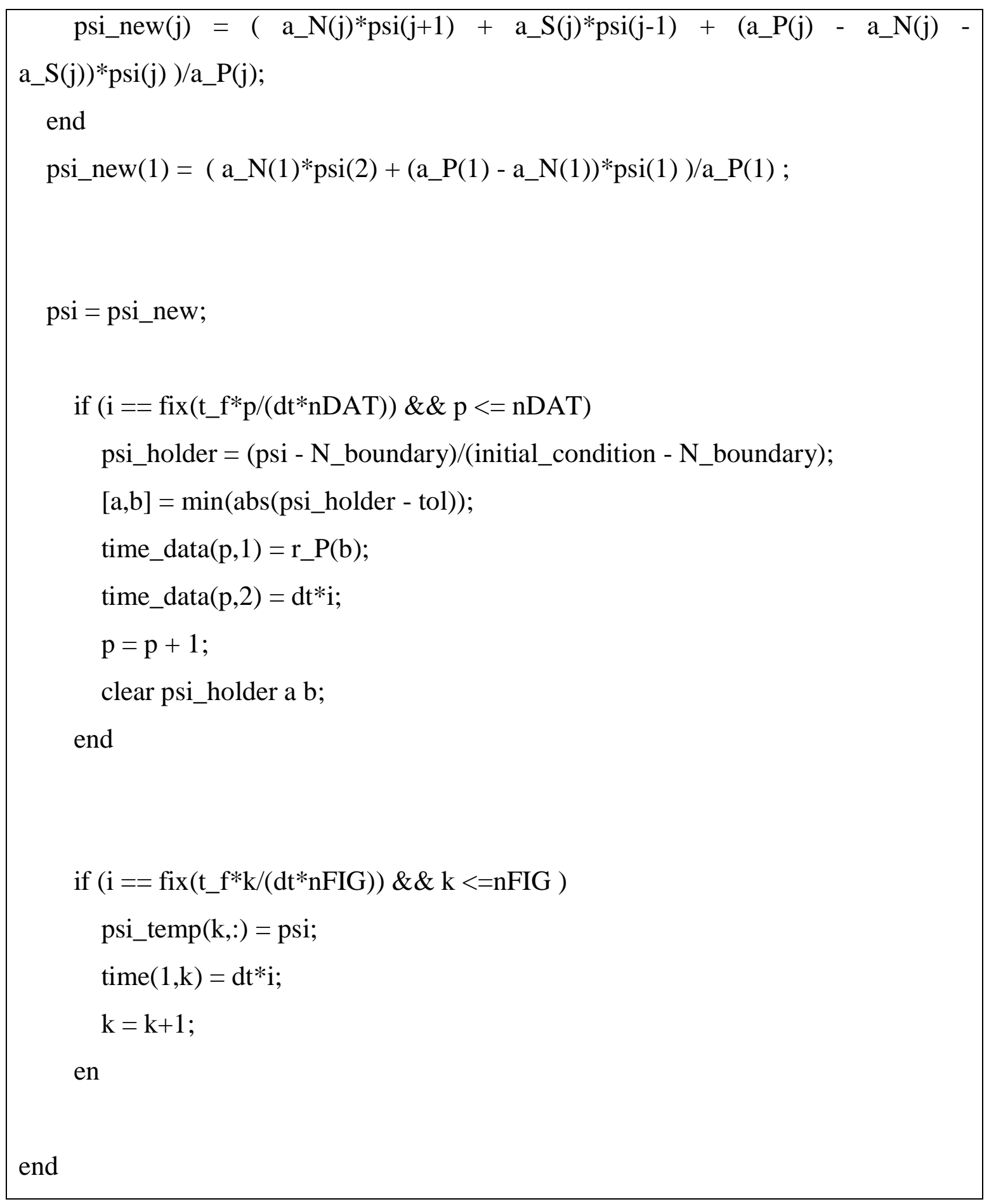




\section{APPENDIX B : DREAM ${ }^{\circledR}$ Code}

\section{What is the DREAM ${ }^{\circledR}$ code?}

The DREAM ${ }^{\circledR}$ code is a transient 3D-Navier-Stokes solver utilizing second order upwind-discretized schemes, accompanied by a fractional step method ${ }^{(20)}$. As it stands, DREAM $^{\circledR}$ is iterative by design and comprises enviable attributes such as being positivedefinite, conservative, and computationally efficient [23].

The DREAM ${ }^{\circledR}$ code has been verified; see sources [24] and [23]. The Implicit Turbulence Model (ITM) method allows for DREAM ${ }^{\circledR}$ to perform very well - in being able to simulate flow for varying geometries; see source [25]. The code was written in FORTRAN $^{\circledR}$ with precise, accurate descriptions regarding the subroutines included in the implementation; a short summary is presented here for the reader's convenience - see Table B-1.

\section{Who implemented the DREAM ${ }^{\circledR}$ code?}

The DREAM ${ }^{\circledR}$ code was developed at West Virginia University under the guidance of Dr. Ismail. B. Celik.

\section{What is the objective of using DREAM ${ }^{\circledR}$ here?}

In this section, results for the microchannel obtained by utilizing the DREAM ${ }^{\circledR}$ code are presented and compared against those obtained from Fluent. The objective here was to demonstrate DREAM ${ }^{\circledR}$ s suitability in generating acceptable results. This activity will add to DREAM ${ }^{\circledR}$ s credibility in being a solid code and help with future developments in the area of performing simulations on microchannels.

\section{Description of Program Files:}

Brief descriptions of the files comprising the DREAM ${ }^{\circledR}$ code are presented in Table B-1, and a flow chart representing the DREAM ${ }^{\circledR}$ code is shown in Figure B-1. It may be noted that the information presented here should be considered very basic. The DREAM ${ }^{\circledR}$ code despite being efficient is fairly complex and has been in development throughout the last decade. The current author is not yet up-to-par with all the intricacies involved in the code and is only familiar with manipulating basic functionalities to suite the relevant geometric boundaries. For further information the reader may refer to sources [24], [25] and [26]. 
Table B-1: Brief component overview of the DREAM $®$ code

\begin{tabular}{|l|l|}
\hline CALC_FLOW.f90 & $\begin{array}{l}\text { This subroutine initiates coffcients for a generalized } \\
\text { transport equation, with additional information regarding } \\
\text { convection and diffusion terms treated implicitly. The } \\
\text { subroutine accounts for the flow field variable Uvel, Vvel } \\
\text { and Wvel. }\end{array}$ \\
\hline BCONS.f90 & $\begin{array}{l}\text { Contains four subroutines for setting implicit and explicit } \\
\text { boundary } \\
\text { conditions of various types. }\end{array}$ \\
\hline CONFIG.f90 & $\begin{array}{l}\text { Contains subroutines to set default variable, read boundary } \\
\text { conditions, and create solution variables }\end{array}$ \\
\hline D3F90.f90 & Entry point of console application. \\
\hline FILEPROCSMOD.f90 & $\begin{array}{l}\text { Track values at a point with time. Subroutines to write } \\
\text { restart file, and TECPLOT recognizable output files for } \\
\text { different for field variables. }\end{array}$ \\
\hline GRIDMOD.f90 & $\begin{array}{l}\text { Subroutines to generate the staggered grid. The grid in the } \\
\text { data files is normalized and does not include the ghost cells. }\end{array}$ \\
\hline IMBDRYMOD.f90 & $\begin{array}{l}\text { Subroutines contain all the immersed boundary method } \\
\text { parameters for different shapes. NOTE: the default } \\
\text { "porosity matrix" is created here. However, for the current } \\
\text { run the porosity matrix was implemented utilizing a } \\
\text { customized MATLAB code. This code will be referred to as } \\
\text { the "HGB porosity code." }\end{array}$ \\
\hline All the program variables are initialized in this file. \\
\hline INCLUDE.f90 & $\begin{array}{l}\text { Function used to interpolate variables at faces and specified } \\
\text { nodal locations. }\end{array}$ \\
\hline INTERPOLATION.f90 \\
\hline PSOLVERS.f90 & $\begin{array}{l}\text { Implements varying solver options available to solve 3D } \\
\text { symmetric matrices. }\end{array}$ \\
\hline UTILITIES.f90 & $\begin{array}{l}\text { Miscellaneous subroutines to perform various subsidiary } \\
\text { functions such as finding variables in an array, calculating } \\
\text { flow-rate and so forth. } \\
\text { specified. Options include inputting the inlet, boundary, and } \\
\text { outlet conditions. The time-step, and information regarding } \\
\text { the output file frequency and type may also be specified. }\end{array}$ \\
\hline
\end{tabular}




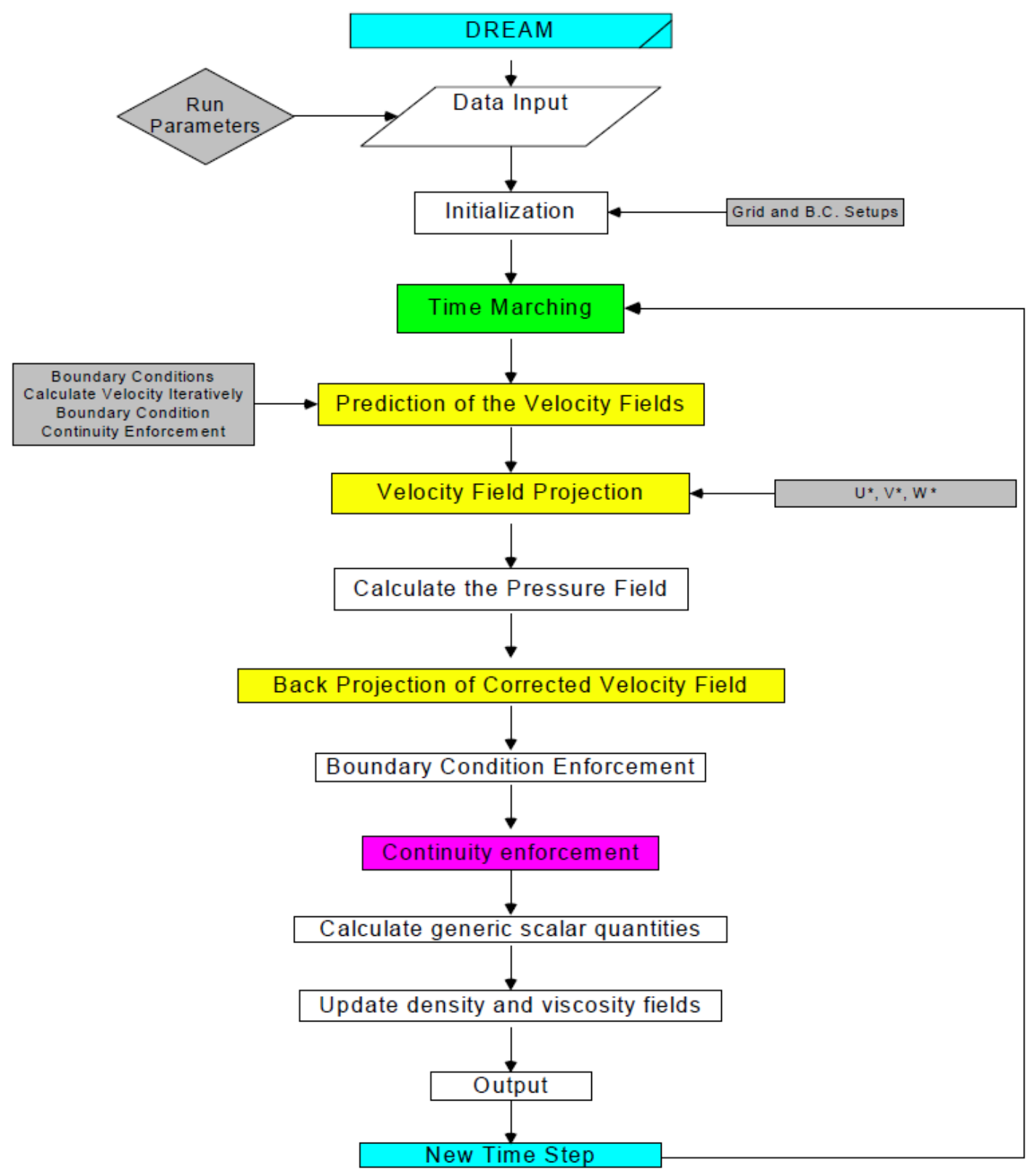

Figure B-1: A brief flow chart of the DREAM ${ }^{\circledR}$ code obtained from [24]. 


\section{Using the DREAM ${ }^{\circledR}$ code:}

Using the DREAM ${ }^{\circledR}$ code to generate results for the microchannel was very straight forward. The boundary conditions had to be altered to match the microchannle. This information was edited into the "start.inp" file - the entire file is attached for the reader convenience; see Table B-4. In addition to specifying the boundary conditions this file also accepts information regarding the discretization-scheme, time-step, output files type/frequency, and various other user desired inputs/outputs - see Table B-1for description.

The boundary conditions that can be specified in "start.inp" are limited to the external flow domain. This limitation does not permit internal walls to be modeled in the geometry. Thanks to the intelligent minds behind conceptualizing $\operatorname{DREAM}^{\circledR}$, a workaround for this purpose was developed. The internal structures in the geometry could be manually modeled as a input recognized by DREAM ${ }^{\circledR}$. This file may be read into the program during the execution (i.e. running the code) and the desired boundaries obtained. This file for the current case was known as "porosity.dat" - it was generated using a customized MATLAB ${ }^{\circledR}$ program - see Table B-3.

The microchannel geometry comprises a sequence of repetitive baffle structures contained within it - see Figure 1-1. Each sequence is termed a cycle ${ }^{[15]}$. The geometry considered suitable for the DREAM ${ }^{\circledR}$ code was one cycle of the HGB channel - see Figure B-2. The entire geometry (consisting of about 7 cycles) could not be modeled as the computational requirements far exceeded the resources available at the lab. It may be noted here that this limitation could be resolved utilizing a "Parallel-Computing" version of DREAM ${ }^{\circledR}$. The current run was processed utilizing a "Serial-Computing" version of $\operatorname{DREAM}^{\circledR}$.

The mesh created by DREAM is structured and closely captures the wall curvature with acceptable tolerance. Owing to using a structured mesh, the wall boundaries are modeled by fine/uniform cubical elements. These cubes give the boundary interface a rugged zig- 
zag pattern; see Figure B-2. By contrast, the mesh used in the fluent run is unstructured and preserves sharp wall boundaries.

Structured and un-structured meshes both have their own set of advantages and disadvantages. The final conclusion depends really on each unique case. For the current case, results obtained from DREAM are presented in the following section - note: brief analyses on the differences between the mesh structures are also presented.

(i)

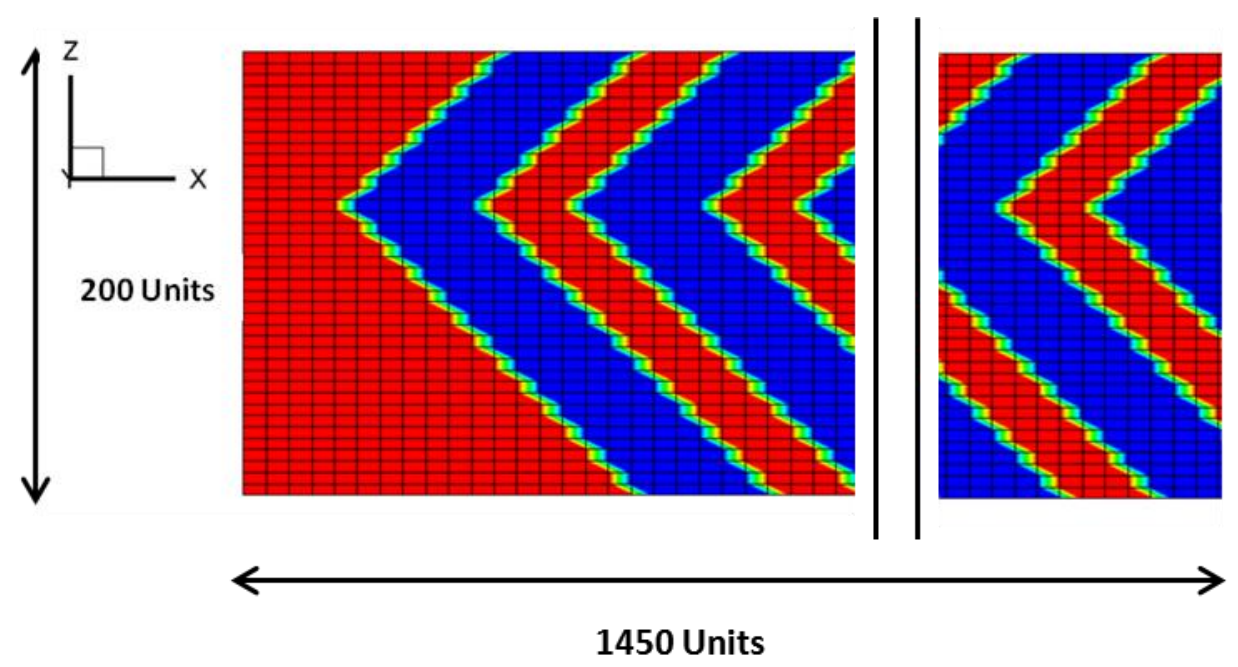

(ii)

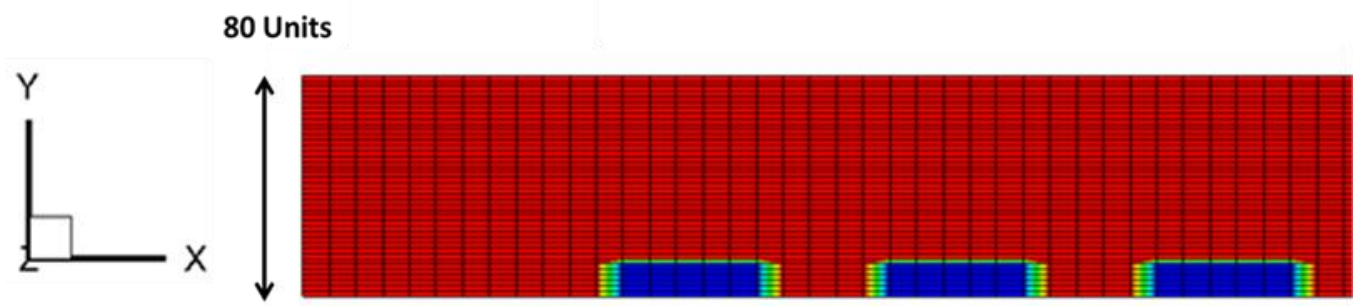

Figure B-2: (i) and (ii) represents the mesh generated by DREAM ${ }^{\circledR}$ 
Table B-2: Difference between Dream and Fluent

DREAM

Fluent

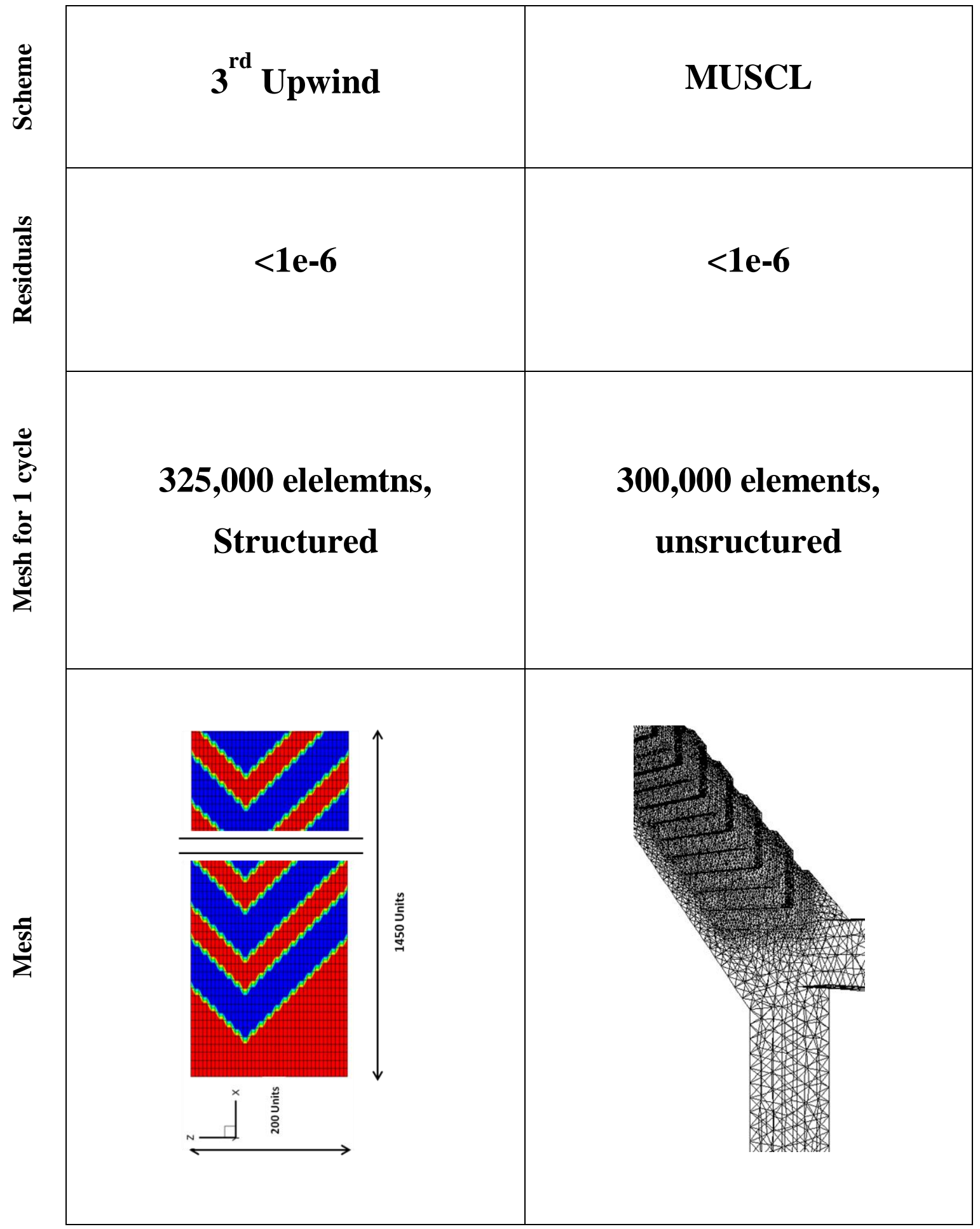


(i)

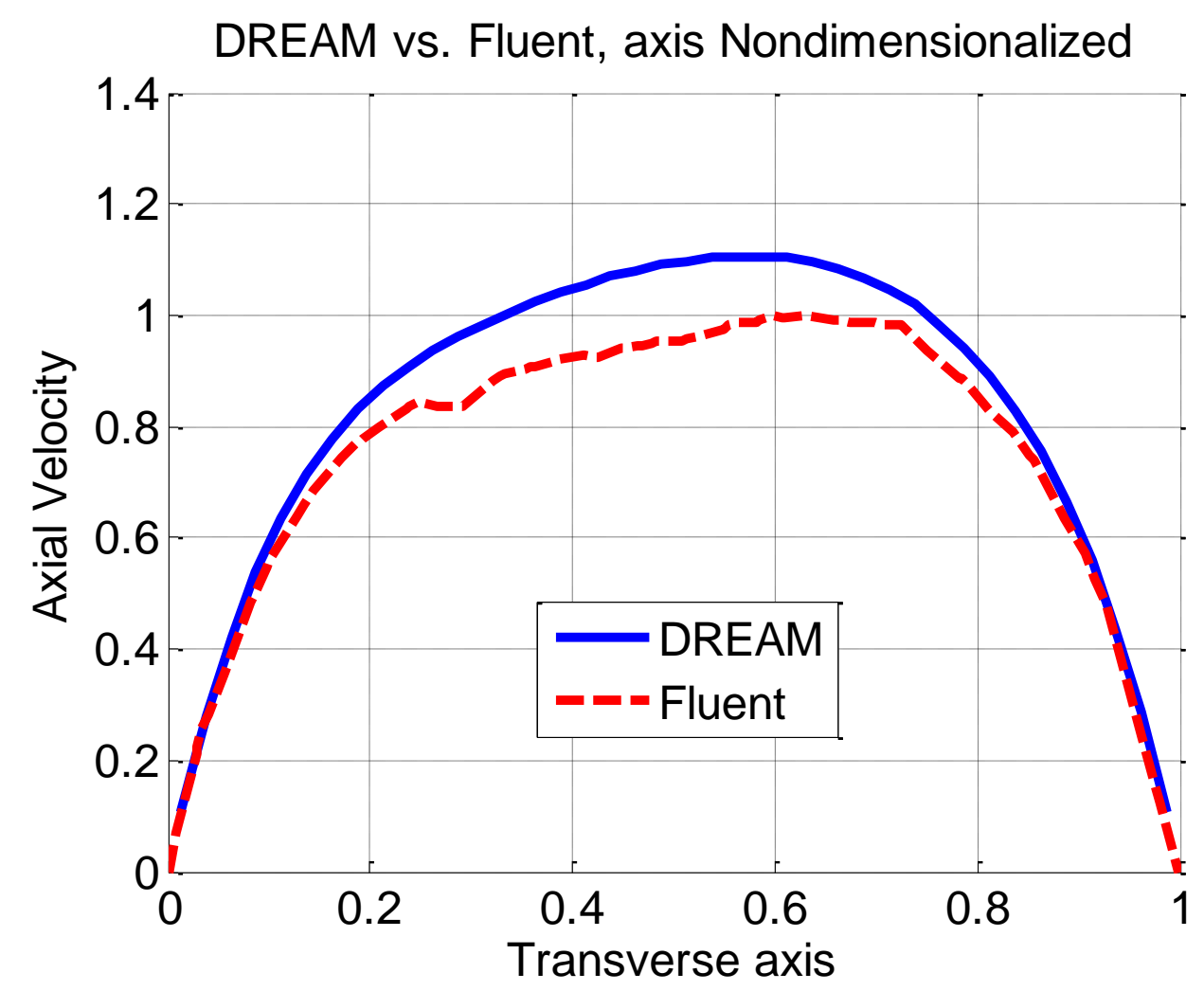

(ii)

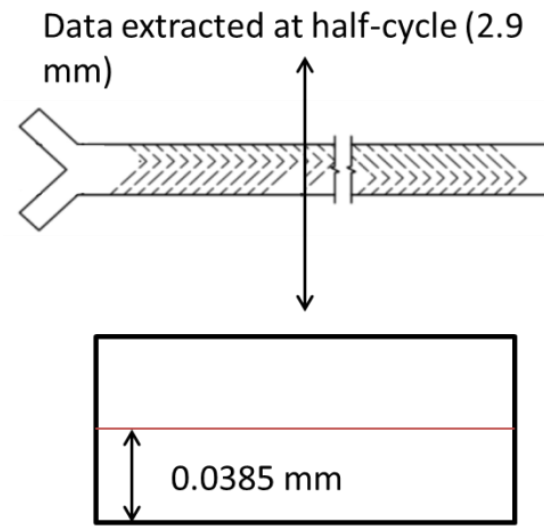

Figure B-3: DREAM vs. Fluent; axial profile 
(i)

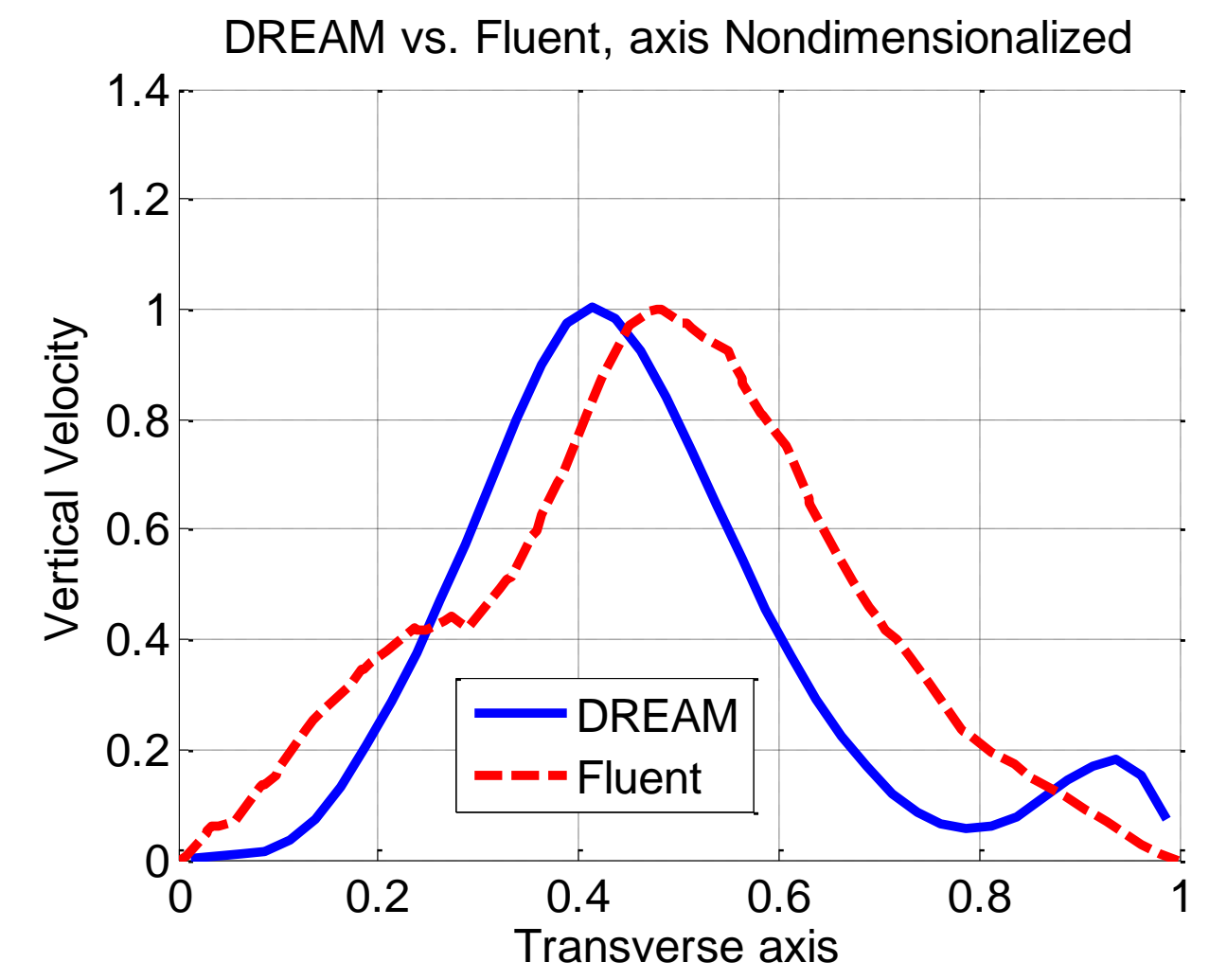

(ii)
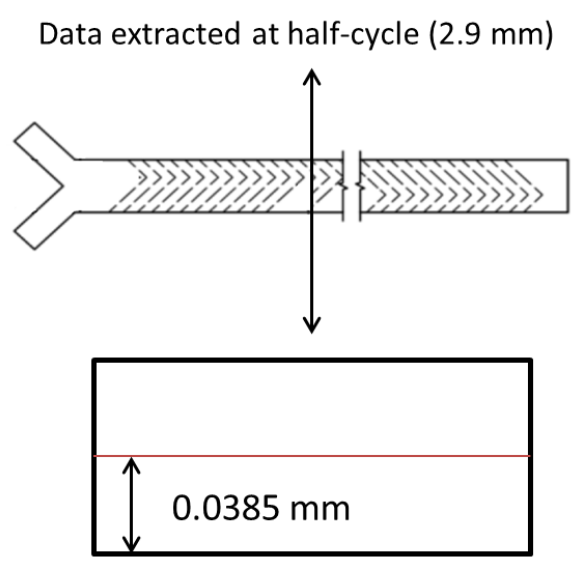

Figure B-4: DREAM vs. Fluent; Vertical Velocity 
(i)

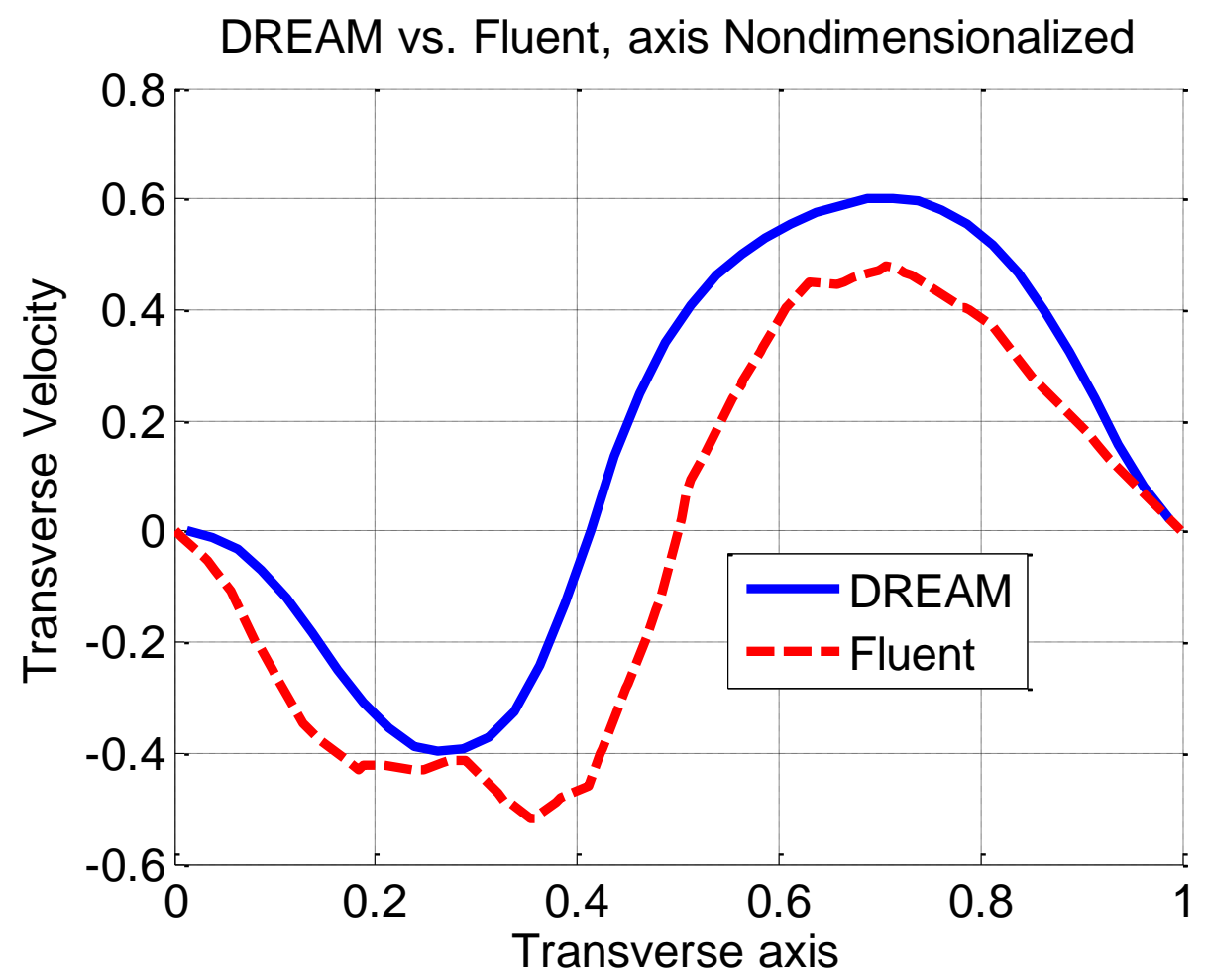

(ii)
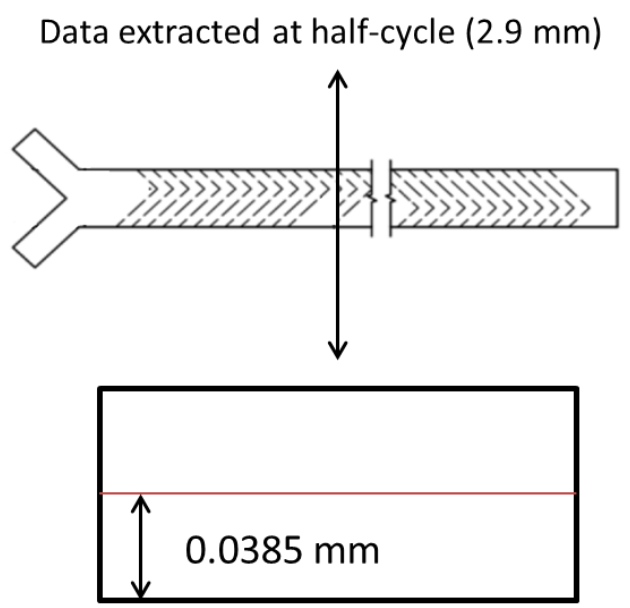

Figure B-5: DREAM vs. Fluent; transverse velocity 
FLUENT : Flow Rate $99.7 \%$ conserved at $2.9 \mathrm{~mm}$

(i)

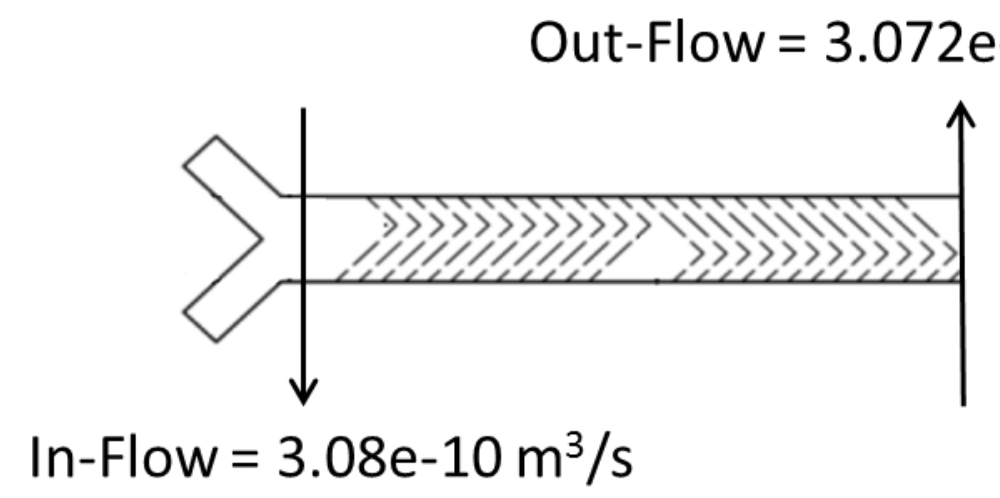

DREAM : Flow Rate $90 \%$ conserved at $2.9 \mathrm{~mm}$

(ii)

Out-Flow $=2.9 \mathrm{e}-3 \mathrm{~m}^{3} / \mathrm{s}$

Figure B-6: DREAM vs. Fluent, flow-rates conservation 


\section{DREAM $^{\circledR}$ vs. Fluent ${ }^{\circledR}$ Results}

In this section the results obtained from DREAM are compared against the results obtained from Fluent. The grid and scheme convergence results for Fluent's solution were already discussed earlier. Hence, comparing DREAM's result against that of Fluent is a productive exercise and will lead to establishing the credibility of the code. Some key differences between DREAM and Fluent are shown in Table B-2.

Prior to discussing the results, it may be noted that the residuals for both cases (DREAM and Fluent) were allowed to approach values lower than 1e-6 for all relevant variables. Additionally, the DREAM code utilized a $3^{\text {rd }}$ order UPWIND scheme to generate results. Hence, it was expected from the onset that the results obtained from DREAM might not exactly agree with those obtained from Fluent.

The velocity profiles for the three components (i,j, and $\mathrm{k}$ ) were extracted about 2.9 $\mathrm{mm}$ downstream. The profile consisted of a transverse running line (extending throughout the width of the channel) positioned $0.0385 \mathrm{~mm}$ above the surface of the channel. The velocity components were extracted from both the solvers (DREAM and Fluent).

As can be seen in Figure B-3, Figure B-4, and Figure B-5, the profile curves are fairly similar. One observation that stands out is the smooth profiles representing DREAM in contrast to the rugged profiles representing the Fluent data. One possible explanation to this observation could be the structures of the mesh used in either case. It was mentioned earlier that the DREAM case utilized a structured grid - see Figure B-2. Also, DREAM's mesh is marginally finer compared to Fluent. Both these factors could potentially aid in generating uniformly distributed data resulting in a smooth profile.

The flow-rate conservation was also analyzed between the DREAM and Fluent results; see Figure B-6. The flow-rate at the inlet was determined from the boundary conditions and used as the reference to assess flow-conservation at downstream cross- 
sections. DREAM indicated 90\% flow-conservation at a cross-section obtained 2.9 mm downstream; see Figure B-6 (ii). Despite appearing reasonably high, there still exists a $10 \%$ loss that could potentially deliver results that might not be within tolerable ranges. On the other hand, Fluent results at the same location indicate superior flow-conservation. From Figure B-6 (i) it can be seen that the Fluent case conserves $99.7 \%$ of the flow. This observation raises a few questions regarding DREAM's potential to function as a stable CFD solver. Unfortunately an in-depth analysis to address this discrepancy is not undertaken owing to being beyond the scope of the current study. Nevertheless, interested readers are encouraged to consider sources [23], [24], and [25].

Despite the differences, the results from DREAM appear very encouraging. Owing to the data sets (specially the velocity field) being reasonably consistent, it is expected that the streamtrace method employed on the DREAM data would generate results comparable to those presented earlier - see section 3.4 on page 28 .

The objective here was to test DREAM's potential in being able to generating acceptable results. Despite not proving to be impeccable, this point of view has definitely been met with enliven conclusions.

The author believes this development to be very promising in terms of further improving DREAM's credibility as a stable and robust CFD solver. 


\section{Table B-3: HGB geometry internal wall code}

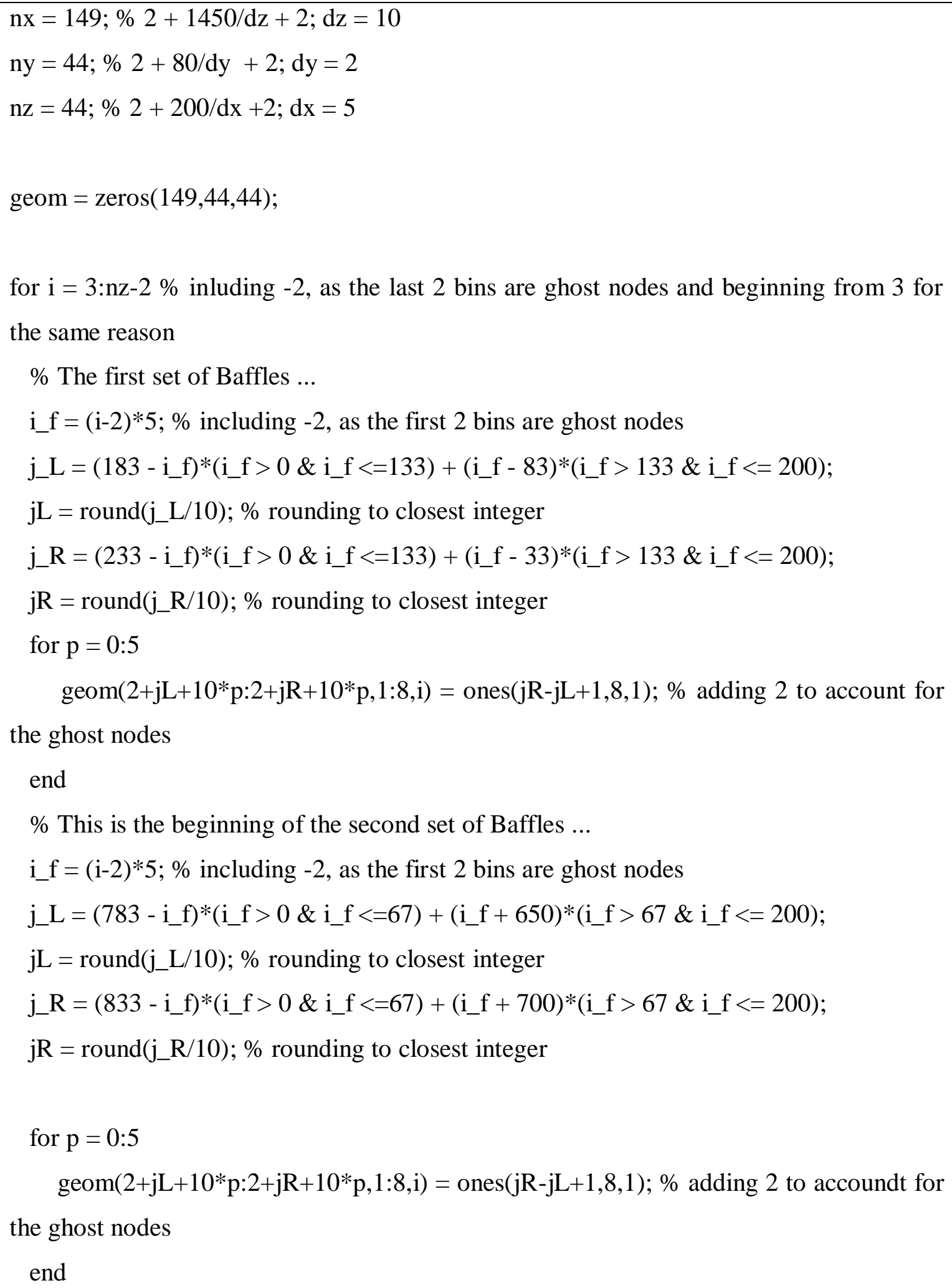




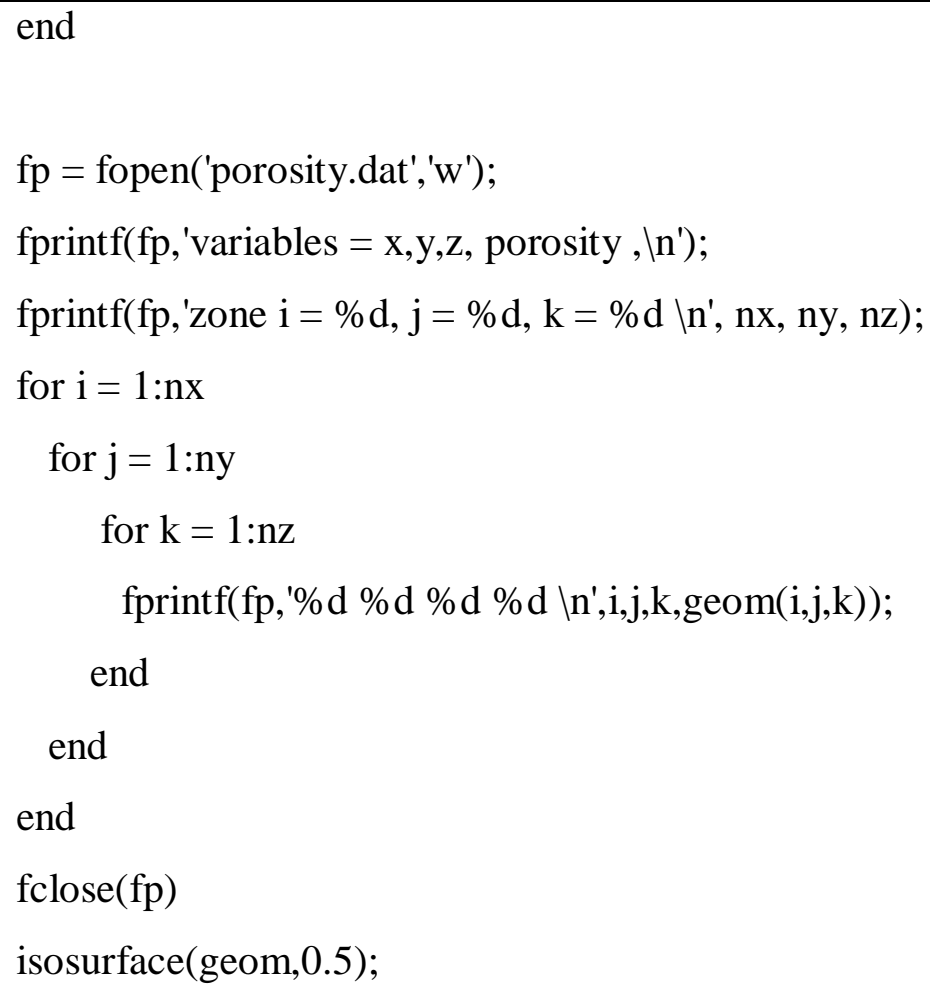




\section{Table B-4: Start.inp}

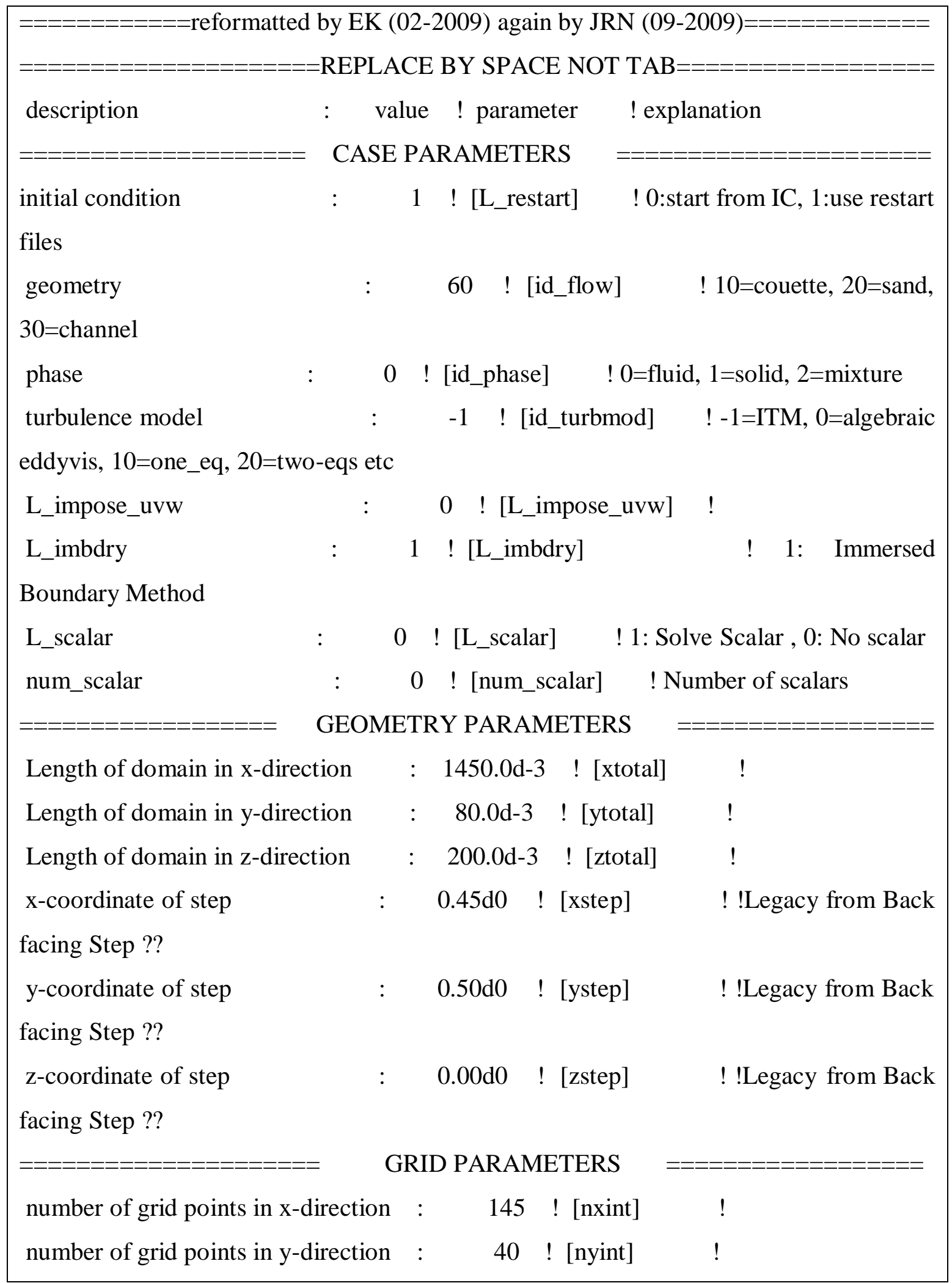




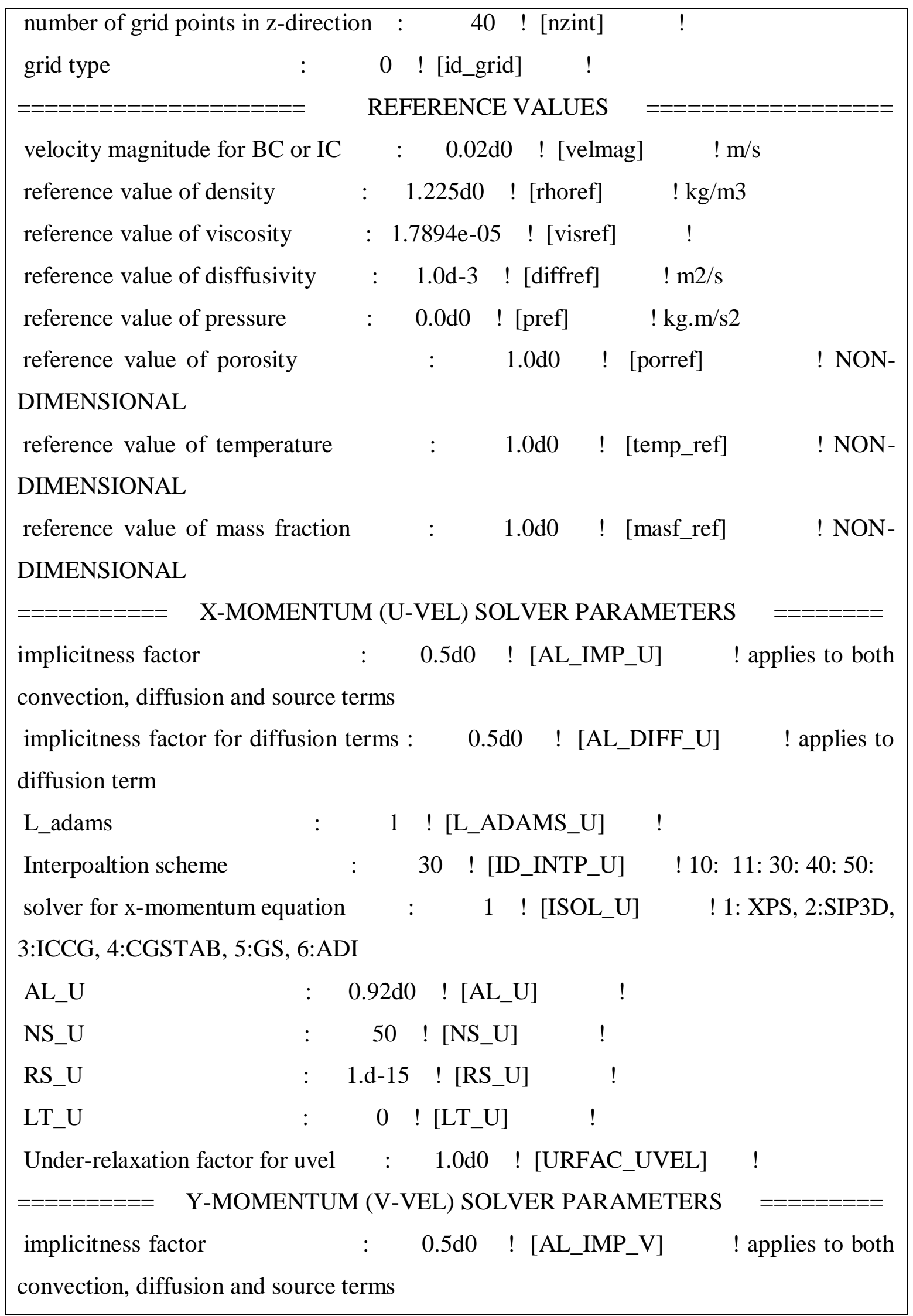




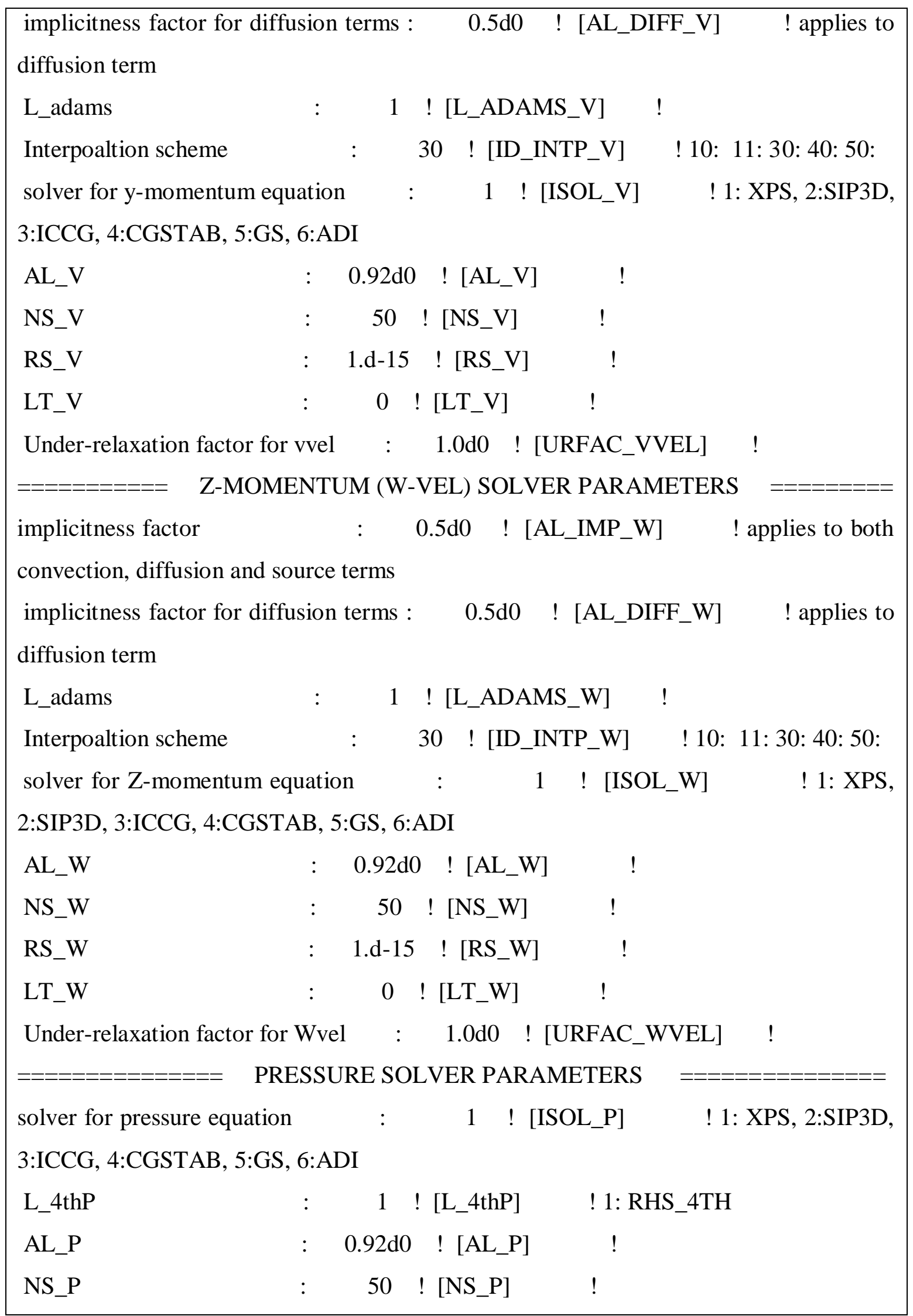




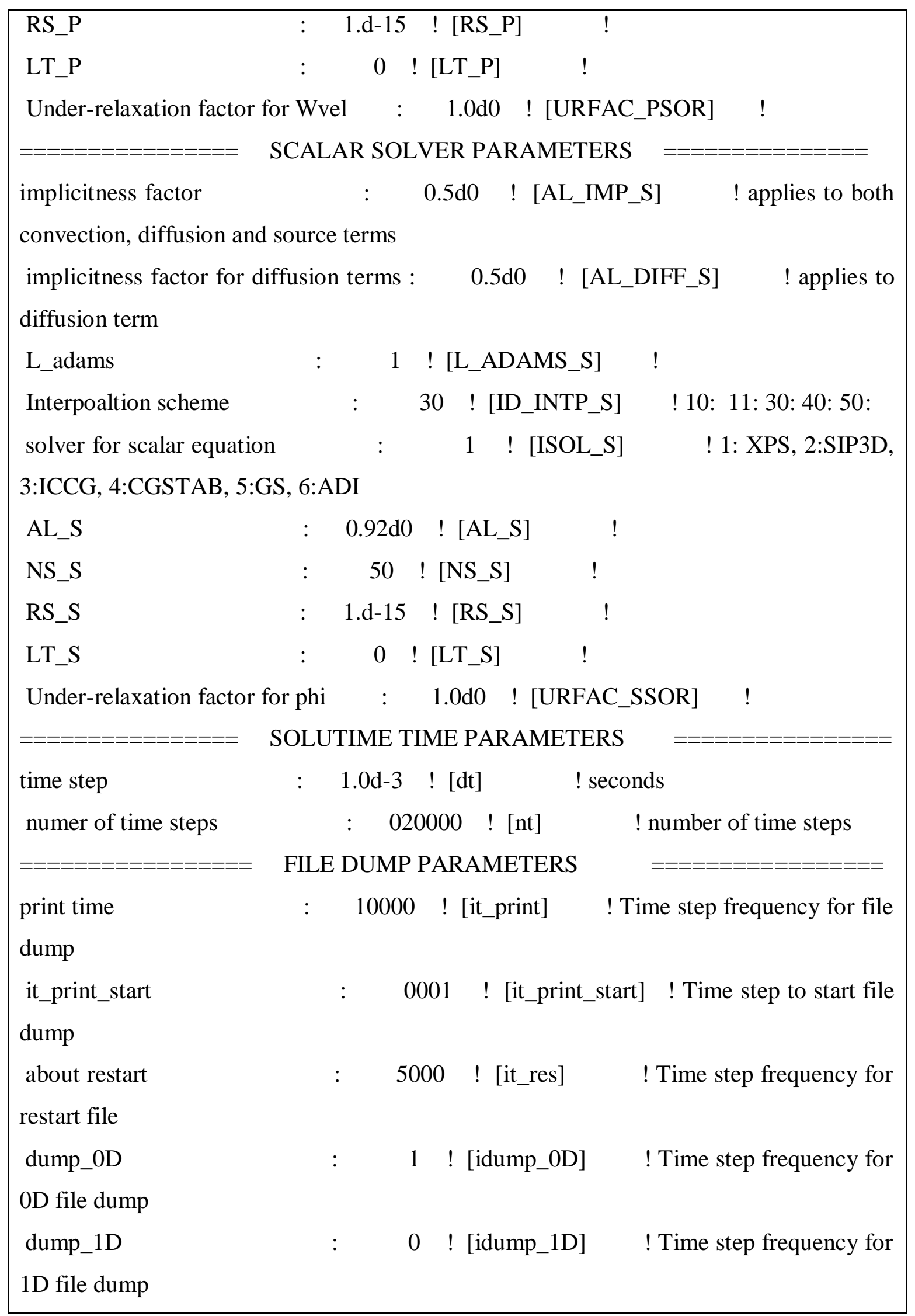




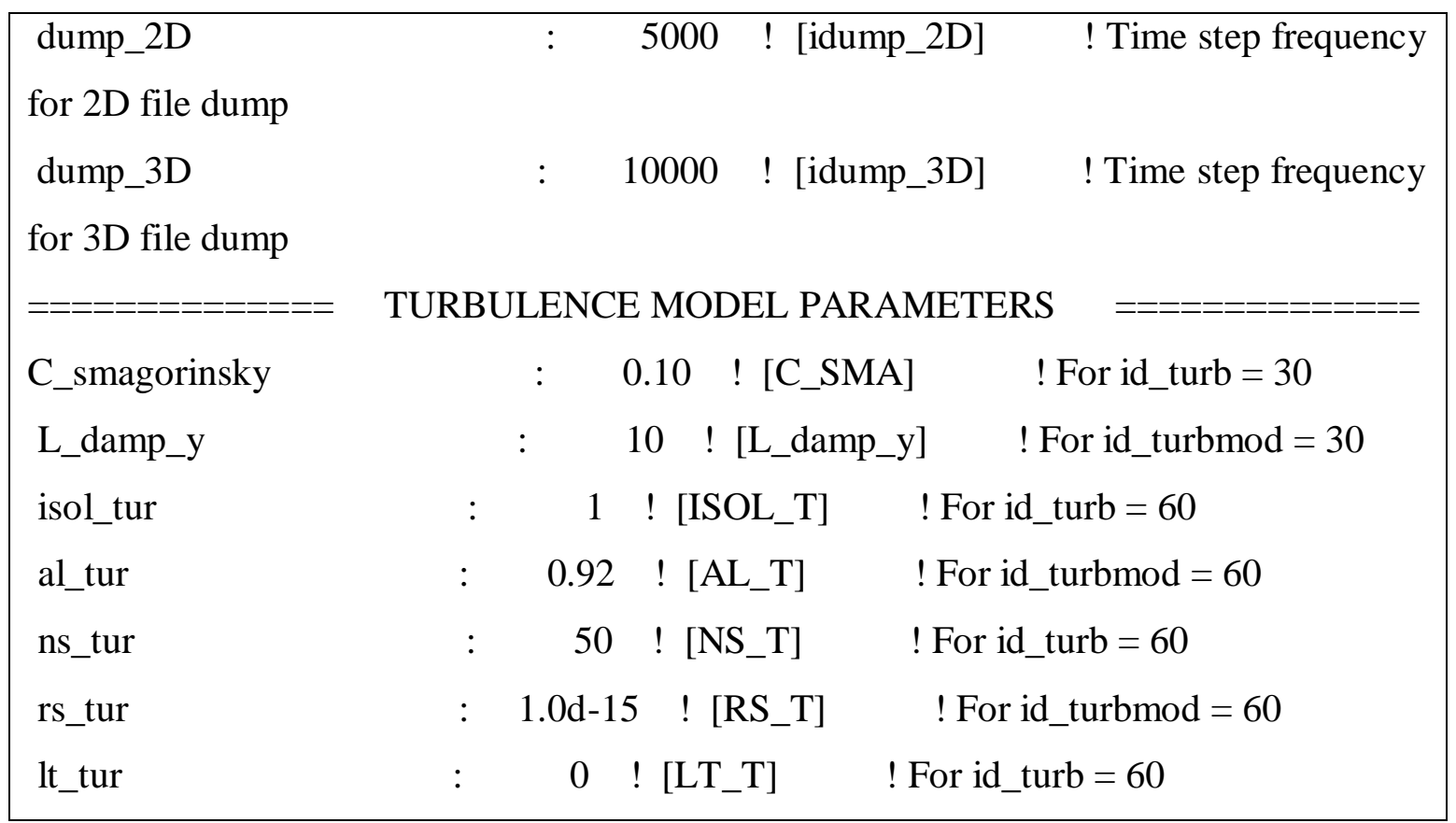




\section{Quantification of Mixing in Microchannels}

\section{Vijaymaran Manickam}

Thesis submitted to the

Benjamin M. Statler

College of Engineering and Mineral Resources

at

West Virginia University

In partial fulfillment of the requirements for the degree of

Master of Science

in

Mechanical Engineering

APPROVAL OF THE EXAMINATION COMMITTEE

Ismail B. Celik, Ph.D., Chair

Yuxin Liu, Ph.D.

Date

Wade Huebsch, Ph.D. 